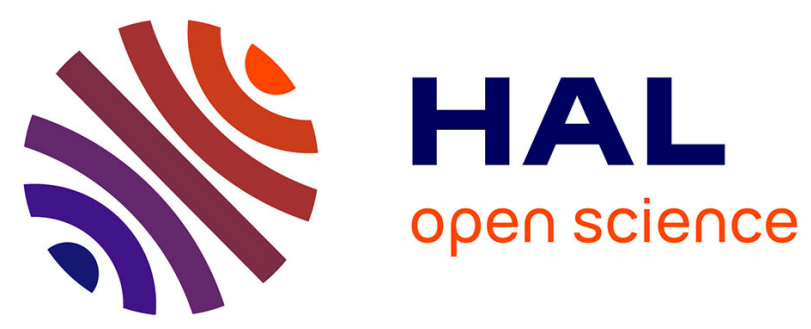

\title{
From Simple Cyclic 1,3-Ketoamides to Complex Spirolactams by Supported Heterogeneous Organocatalysis with PS-BEMP
}

Aouicha Benmaati, Hadjira Habib Zahmani, Salih Hacini, José Carlos Menéndez, Xavier Bugaut, Jean Rodriguez, Thierry Constantieux

\section{To cite this version:}

Aouicha Benmaati, Hadjira Habib Zahmani, Salih Hacini, José Carlos Menéndez, Xavier Bugaut, et al.. From Simple Cyclic 1,3-Ketoamides to Complex Spirolactams by Supported Heterogeneous Organocatalysis with PS-BEMP. Synthesis: Journal of Synthetic Organic Chemistry, 2016, 48 (19), pp.3217 - 3231. 10.1055/s-0035-1561485 . hal-01415069

\author{
HAL Id: hal-01415069 \\ https://hal.science/hal-01415069
}

Submitted on 12 Dec 2016

HAL is a multi-disciplinary open access archive for the deposit and dissemination of scientific research documents, whether they are published or not. The documents may come from teaching and research institutions in France or abroad, or from public or private research centers.
L'archive ouverte pluridisciplinaire HAL, est destinée au dépôt et à la diffusion de documents scientifiques de niveau recherche, publiés ou non, émanant des établissements d'enseignement et de recherche français ou étrangers, des laboratoires publics ou privés. 


\section{From Simple Cyclic 1,3-Ketoamides to Complex Spirolactams by Supported Heterogeneous Organocatalysis with PS-BEMP}

\author{
Aouicha Benmaati \\ Hadjira Habib Zahmania \\ Salih Hacini ${ }^{a}$ \\ José Carlos Menéndez ${ }^{\mathbf{b}}$ \\ Xavier Bugaut ${ }^{c}$ \\ Jean Rodriguez ${ }^{* c}$ \\ Thierry Constantieux*c \\ a Laboratoire de Chimie Fine, Faculté des Sciences Exactes et \\ Appliquées, Université d'Oran-1, Ahmed Benbella BP-1524- \\ Menouar, 31000 Oran, Algeria

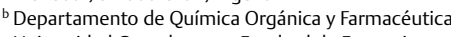 \\ Universidad Complutense, Facultad de Farmacia, \\ Universidad Complutense, 28040 Madrid, Spain \\ c Aix-Marseille Université, Centrale Marseille, CNRS iSm2 \\ UMR 7313, 13397, Marseille, France \\ jean.rodriguez@univ-amu.fr \\ thierry.constantieux@univ-amu.fr \\ In the memory of Professor Jean Normant
}

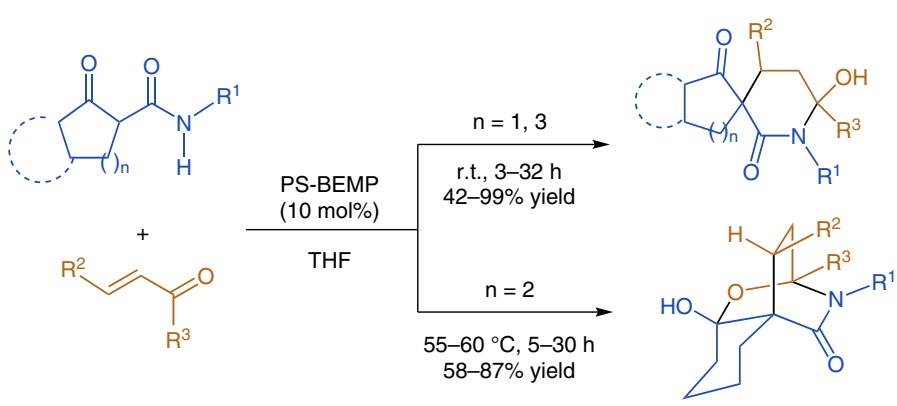

Abstract The reaction between cyclic 1,3-ketoamides and Michael acceptors in the presence of a catalytic amount of a polymer-supported organobase PS-BEMP has been developed for a direct access to spirocyclic 1,3-ketolactams through a domino Michael addition/hemiacetalization sequence. The products could be isolated in high chemical yields and purities after simple filtration, and the catalyst could be re-used without any re-activation. These spirolactams, containing a hemiaminal moiety, may be viewed as precursors of $\mathrm{N}$-acyliminium intermediates upon Lewis acid activation, which allowed various subsequent functionalizations leading to original polycyclic lactams.

Key words supported organobase, Michael addition, spirolactam, heterogeneous organocatalysis, $\mathrm{N}$-acyliminium intermediate

In the context of sustainable chemistry, the use of heterogeneous catalysts offers several advantages compared to homogeneous catalysis, notably in terms of isolation of the product, ease of handling, and recycling of the catalyst, ${ }^{1}$ and to some extent, applications to automated systems and flow chemistry. ${ }^{2}$ For several years, various catalytic species have been anchored to diverse insoluble supports, including some chiral organocatalysts for enantioselective synthesis. $^{3}$ The interest for polymer-supported organobases such as bicyclic guanidines (PS-TBD), ${ }^{4}$ phosphines (PS$\left.\mathrm{PPh}_{3}\right)^{5}$ or phosphazenes (PS-BEMP)] ${ }^{6}$ (Figure 1) started at the end of the nineties, essentially for the alkylation of amine derivatives. ${ }^{7}$ Within this field of research, in 2004 we were the first group to report on the use of PS-BEMP as a heterogeneous catalyst for Michael additions. Indeed, this commercially available, user-friendly, and reusable chemical was found to promote the addition of 1,3-dicarbonyls onto various acceptors with high efficiency (Scheme 1, a). ${ }^{8}$ Later on, several other groups applied this strategy to various synthetic methodologies, ${ }^{9}$ including conjugate additions, ${ }^{10}$ ring-opening of epoxides, ${ }^{11}$ Henry reactions, ${ }^{12}$ and hydrophosphonylation of aldehydes. ${ }^{13}$

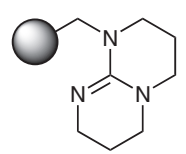

PS-TBD

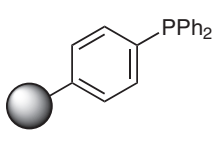

PS-TPP

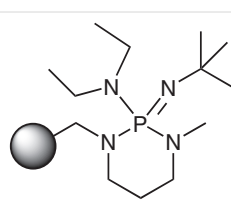

PS-BEMP
Figure 1 Main polymer supported organobases

Remarkable contributions from the groups of Lectka and Dixon also demonstrated that the use of catalytic amounts of PS-BEMP can be cleverly combined with various others catalysts in sequential or domino reactions for the highly stereoselective synthesis of complex molecules. ${ }^{14}$

In the course of our studies on new domino and multicomponent reactions from 1,2- and 1,3-dicarbonyls for the synthesis of polyfunctionalyzed heterocycles, ${ }^{15}$ we focused our interest on the use of 1,3-ketoamides. Indeed, compared with the more classical and intensively used 1,3-keto esters and 1,3-diketones containing different nucleophilic and electrophilic reactive sites, the amide moiety can be viewed as a supplementary aza-nucleophilic function, of- 
a) Previous work

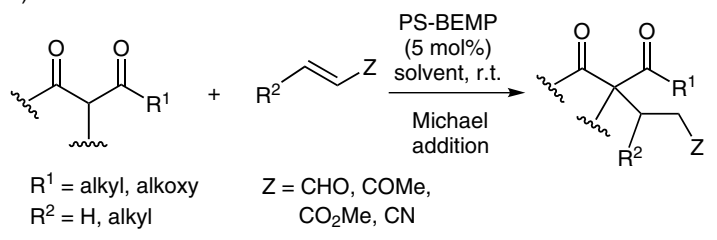

b) This work

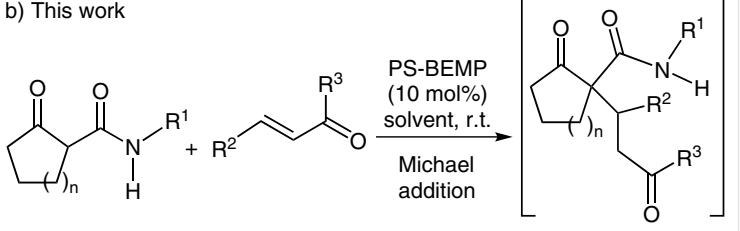

$\mathrm{R}^{1}=\mathrm{H}$, alkyl, aryl $\quad \mathrm{R}^{2}=\mathrm{H}$, alkyl

$\mathrm{n}=1-3 \quad \mathrm{R}^{3}=\mathrm{H}, \mathrm{CO}_{2} \mathrm{Me}$

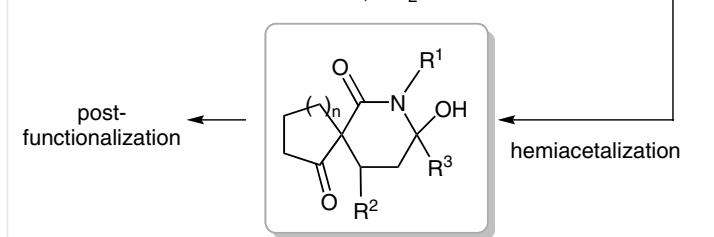

Scheme 1 PS-BEMP catalyzed Michael addition

fering new opportunities for domino reactions. Thus, in the PS-BEMP-catalyzed Michael addition, a suitably substituted secondary 1,3-ketoamide should offer a direct access to ketospirolactams through a domino Michael addition-hemiacetalization sequence (Scheme 1, b). In this formal [3+3] cyclization process, the 1,3-ketoamide reacts as 1,3-bisnucleophile with the Michael acceptor acting as 1,3-biselectrophile.

The spirocyclic 1,3-ketolactam scaffold is frequently associated with various biological activities, including action on cerebrovascular disorders, ${ }^{16}$ anticancer, ${ }^{17}$ antimicrobi$\mathrm{al}^{18}$ and antifungal properties. ${ }^{19}$ Consequently, owing to the great biological and synthetic importance of this heterocyclic core, ${ }^{20}$ the synthesis of spirolactam derivatives has long been an area of intense development, ${ }^{21}$ and still constitutes an active domain of research. In 2000, Cossy and co-workers identified cyclic 1,3-ketoamides as potential substrates for this purpose, as illustrated by their publication on the Mn(III)-induced radical cyclization of unsaturated 1,3-ketocarboxamides. ${ }^{22}$ Later, gold- and palladiumbased catalysts have also been used for the intramolecular addition of 1,3-ketoamides to alkenes ${ }^{23}$ and allenes, ${ }^{24}$ respectively. As reported by our team and by Marini's group, $\mathrm{DBU}^{25}$ or N-heterocyclic carbenes ${ }^{26}$ can alternatively promote Michael addition or alkylation of 1,3-ketoamides as key steps in sequences giving access to spirocyclic-1,3-ketolactams.
In this paper, we report a direct access to spirocyclic1,3-ketolactams from easily accessible simple cyclic 1,3-ketoamides and Michael acceptors, using a catalytic amount of the commercially available polystyrene-supported organobase PS-BEMP. This methodology avoids the use of metal-based catalysts and harsh reactions conditions, and allows an easy isolation of the product from the re-usable catalyst. Post-functionalizations of the spirocyclic-1,3-ketolactams have been also studied, exploiting the reactivity of $\mathrm{N}$-acyliminium intermediates generated from the hemiaminal moiety.

As illustrated in Table 1, some preliminary experiments confirmed our working hypothesis concerning the possibility of directly accessing spirocyclic 1,3-ketolactams from simple substrates, using a catalytic amount of a supported organobase. We started our study by reacting $N$-tosyl-1,3ketoamide 1a with 1.1 equivalent of acrolein (2a) in THF at room temperature in the presence of $10 \mathrm{~mol} \%$ of PS-BEMP. Pleasingly, the desired spiro[4.5]decane ketolactam 3a was formed as the only product and isolated by simple filtration in high yield as a 1.5:1 mixture of two diastereomers (Table 1, entry 1 ). Moreover, product purity was higher than $90 \%$. In order to compare the efficiency of PS-BEMP with more classical mineral or organic bases, complementary experiments were run in standard conditions. A heterogeneous catalyst such as potassium carbonate (entry 2 ) or a homogeneous one such as DBU, BEMP, or guanidine (entries 3-5) afforded a mixture of the desired product 3aa and bicyclic ketone 4aa resulting from an intramolecular aldolization of the Michael adduct, ${ }^{27}$ together with unidentified products. On the contrary, use of triphenylphosphine or its resin-supported version (entries 6 and 7) led to the formation of the desired product 3aa with an efficiency and simplicity of use similar to PS-BEMP. However, $\mathrm{PPh}_{3}$ performed less convincingly when $N$-allyl-1,3-ketoamide $\mathbf{1 b}$ was used as substrate, leading to a 1:1 mixture of 3ba and $\mathbf{4 b a}$ (entries 8 and 9). In sharp contrast, we were pleased to note that the PS-BEMPcatalyzed reaction of $\mathbf{1 b}$ with acrolein afforded exclusively the desired spiro compound in high yield after simple filtration (entry 10).

These preliminary results confirmed the relevance of using a supported organobase to reach our synthetic goal. Thus, we started to evaluate the scope of the reaction between various secondary 1,3-ketoamides $\mathbf{1}^{28}$ and acceptors $\mathbf{2}$ in the presence of a catalytic amount of PS-BEMP, applying the previously established standard conditions (Scheme 2 and Scheme 3). Initially, the reactions between acrolein (2a) and $\mathrm{N}$-homoallyl- and $\mathrm{N}$-o-bromobenzyl-1,3-ketoamides 1c and 1d were investigated and found to afford after stirring at room temperature during 24 hours the corresponding spirobicyclic-1,3-ketolactams 3ca and 3da in good to excellent isolated yields, as a 1:1 mixture of two diastereomers. In the same conditions, 1,3-ketoamide 1e, derived from 2-furylmethanamine, when treated with ei- 


\begin{tabular}{|c|c|c|c|c|c|}
\hline \multicolumn{2}{|c|}{$\begin{array}{c}1 \\
1 \mathrm{a} R=\mathrm{Ts} \\
\text { 1b } \mathrm{R}=\text { allyl }\end{array}$} & \multicolumn{2}{|c|}{ 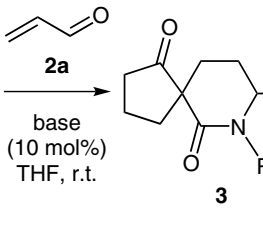 } & $\mathrm{R}$ & \\
\hline Entry & Base & Substrate & Time $(h)^{b}$ & Product & Yield (\%) \\
\hline 1 & PS-BEMP & $1 a$ & 3 & $3 a a$ & 94 \\
\hline 2 & $\mathrm{~K}_{2} \mathrm{CO}_{3}{ }^{\mathrm{d}}$ & $1 \mathbf{a}$ & 16 & \multicolumn{2}{|c|}{ complex mixture } \\
\hline 3 & DBU & $1 \mathbf{a}$ & 16 & \multicolumn{2}{|c|}{ complex mixture } \\
\hline 4 & BEMP & $1 \mathrm{a}$ & 3 & \multicolumn{2}{|c|}{ complex mixture } \\
\hline 5 & guanidine & $1 \mathrm{a}$ & 3 & \multicolumn{2}{|c|}{ complex mixture } \\
\hline 6 & $\mathrm{PPh}_{3}$ & $1 \mathrm{a}$ & 3 & $3 a a$ & 90 \\
\hline 7 & $\mathrm{PS} \mathrm{PPh}_{3}$ & $1 \mathrm{a}$ & 3 & 3aa & 92 \\
\hline 8 & $\mathrm{PPh}_{3}$ & $1 b$ & 24 & $3 b a+4 b a^{e}$ & 85 \\
\hline 9 & $\mathrm{PS}^{-\mathrm{PPh}_{3}}$ & $1 b$ & 24 & $3 b a+4 b a^{e}$ & 91 \\
\hline 10 & PS-BEMP & $1 b$ & 24 & 3ba & 87 \\
\hline
\end{tabular}

a Reaction conditions: 1,3-ketoamide $\mathbf{1}(1.0 \mathrm{mmol})$, acrolein (2a; 1.1 $\mathrm{mmol}$ ), and base (10 mol\%) were mixed in anhyd THF ( $0.5 \mathrm{M}$ ) at r.t. ${ }^{b}$ Reaction time for total conversion of substrate, monitored by TLC. c Isolated yield of product after simple filtration, obtained as a 1.5:1 mixture of diastereomers.

${ }^{d}$ Base used: 20 mol\%.

e Ratio of $3 \mathbf{b a} / \mathbf{4 b a}=1: 1$

ther crotonaldehyde (2b) or 3-(2-furyl)acrolein (2c) led to the formation of the desired products $3 \mathbf{e b}$ and $\mathbf{3 e c}$, respectively, in almost quantitative yields and high chemical purity after simple filtration. Michael acceptor 2d, derived from pyruvic acid, was found to be highly reactive in this sequence, leading quantitatively to 3ed in only 5 hours at room temperature. This Michael acceptor allowed also the use of a less reactive $\mathrm{N}$-aryl-1,3-ketoamide $\mathbf{1 f}$, allowing the formation of product 3fd in $98 \%$ yield albeit after a prolonged reaction time $(32 \mathrm{~h}) .{ }^{29}$ Interestingly, primary 1,3-ketoamide $\mathbf{1 g}$ turned out to be less efficient, but provided 3gd with a free $\mathrm{NH}$ bond in $42 \%$. Seven-membered cyclic 1,3-ketolactam $\mathbf{1 h}$ gave also the desired product 3 hd in high yield, opening up a route to the spiro[5.6]dodecane family, for which only few synthetic methods have been reported to date. ${ }^{14 c, 23 a}$ Finally, substrates $\mathbf{1 i}-\mathbf{k}$, containing a 1-indanone or a 1-tetralone ketoamide moiety, led to the desired spiro[4.5]decane 3ia and spiro[5.5] undecanes 3ja and 3ka products, respectively, in good to excellent isolated yields. Although some products were obtained as a mixture of up to four diastereomers, ${ }^{30}$ one major isomer was preferentially formed and could be systematically isolated and characterized. In the case of product 3ed, the relative configuration of the major diastereomer was established by X-ray crystal structure analysis ${ }^{31}$ (Figure 2).

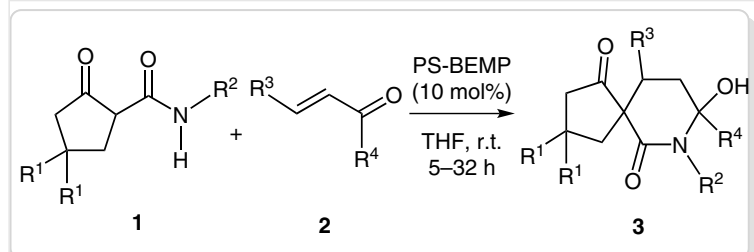

$$
\begin{array}{ll}
\text { 1a } R^{1}=H, R^{2}=T s & \text { 1e } R^{1}=H, R^{2}=\text { 2-furylmethyl } \\
\text { 1b } R^{1}=H, R^{2}=\text { allyl } & \text { 1f } R^{1}=H, R^{2}=P h \\
\text { 1c } R^{1}=H, R^{2}=\text { homoallyl } & \text { 1g } R^{1}=M e, R^{2}=H \\
\text { 1d } R^{1}=H, R^{2}=o \text {-Br-benzyl } &
\end{array}
$$<smiles>[R]NC(=O)C1CCCCCC1=O</smiles><smiles>[R]N([2H])C(=O)C1Cc2ccccc2C1=O</smiles><smiles>[R]NC(=O)C1CCc2ccccc2C1=O</smiles>

1h $\left(R^{2}=2\right.$-furylmethy $)$

$1 \mathbf{i}\left(R^{2}=\right.$ allyl $)$

1j $\left(R^{2}=P h\right)$

1k $\left(R^{2}=\right.$ allyl $)$<smiles>C/C=C/C=O</smiles><smiles>O=C/C=C/c1ccco1</smiles><smiles>COC(=O)/C=C/c1ccccc1</smiles>

Scheme 2 PS-BEMP catalyzed Michael addition/hemiaminalization sequence for the synthesis of spiroketolactams 3
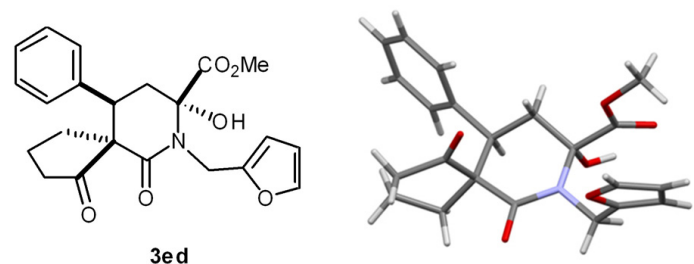

Figure 2 X-ray crystal structure analysis of the major diastereomer of 3ed

Six-membered alicyclic 1,3-ketoamides $\mathbf{1 l}\left(\mathrm{R}^{1}=\mathrm{Ph}\right), \mathbf{1} \mathbf{m}$ $\left(\mathrm{R}^{1}=2\right.$ - furylmethyl $)$, and $\mathbf{1 n}\left(\mathrm{R}^{1}=\right.$ benzyl $)$ adopted a different behavior and in these cases the previous domino sequence was followed by an intramolecular hemiacetalization, leading to complex but stable and isolable tricyclic products $\mathbf{5}$ incorporating an original 2,6-oxazabicyclo[2.2.1] heptane core (Scheme 4). As already discussed for the case of compounds $\mathbf{3}$, the major diastereomer of products $\mathbf{5}$ could be systematically isolated. Interestingly enough, compound $\mathbf{5 n c}$ was formed with a good diastereoselectivity of 10:1. The structures of these tricyclic hemiacetals have been clearly confirmed by X-ray analysis ${ }^{31}$ of a single crystal of product 5ld, as depicted in Figure 3. 


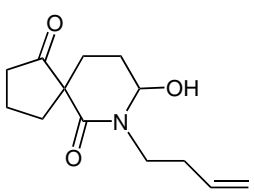

3ca $88 \%($ dr $1: 1)$

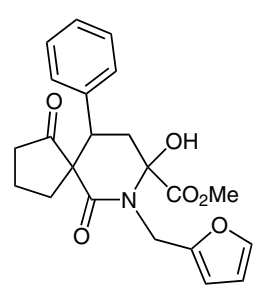

3ed $98 \%(\operatorname{dr} 5: 1.5: 1: 1)^{\mathrm{a}}$<smiles>O=C1CCCC12CCC(O)N(Cc1ccccc1Br)C2=O</smiles>

3 da 98\% (dr 1:1)<smiles>CC(=O)OC1(O)CC(c2ccccc2)C2(CCCC2=O)C(=O)N1c1ccccc1</smiles>

3 fd

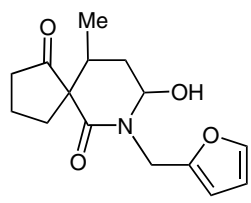

3eb $98 \%(\operatorname{dr} 6: 4: 1: 1)$

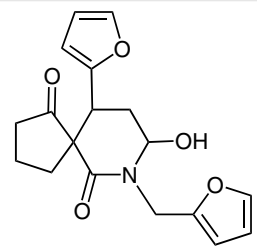

3ec<smiles>CC(=O)N1CC(O)(C(C)=O)CC(c2ccccc2)C12CC(C)(C)CC2=O</smiles><smiles>C=CCN1C(=O)C2(CCC1O)Cc1ccccc1C2=O</smiles>

3ia<smiles>O=C1c2ccccc2CCC12CCC(O)N(c1ccccc1)C2=O</smiles>

3ja 3gd

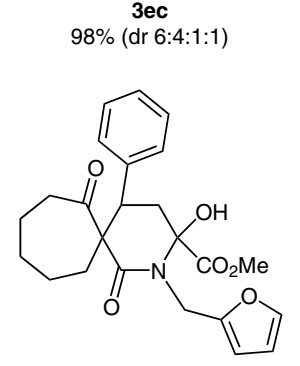

3hd

$88 \%(\text { dr } 3: 1.5: 1.5: 1)^{c}$

$\sim \mathrm{OH}$<smiles>C=CCN1C(=O)C2(CCc3ccccc3C2=O)CCC1O</smiles>

Scheme 3 Scope of the reaction for the synthesis of spirocyclic-1,3-ketolactams 3. Reagents and conditions: 1,3-ketoamide 1 (1.0 mmol), Michael

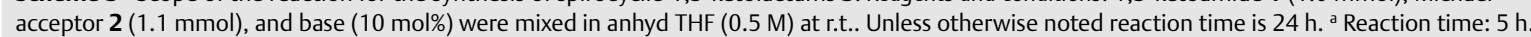
${ }^{b}$ Reaction time: 32 h. ${ }^{c}$ Reaction time: 9 h.
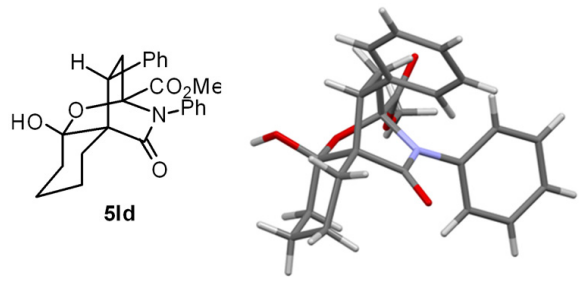

Figure 3 X-ray crystal structure analysis of hemiacetal 5ld

As a final refinement of the evaluation of the scope of our reaction we have run some experiments that clearly show the reusability of the catalyst (Table 2 ). Indeed, by systematically re-using the same sample of catalyst without any specific activation, we repeated several times the reaction between substrate $\mathbf{1 a}$ and acrolein $(\mathbf{2 a})$, which initially led to the desired product 3aa in $94 \%$ yield. For this study, we used a nondestructive rotatory stirring in order to limit degradation of the solid catalyst by mechanical stirring. PS-BEMP was isolated from each experiment by simple filtration and washing with THF, and re-used in the next one. Although it is necessary to increase the reaction time in each re-use of the catalyst to obtain complete conversion, the product is consistently obtained with the same efficiency in the first three recycles. Beyond the fifth use, performance starts to decline, reflecting a gradual deterioration of the catalyst, but isolated yields remain high.

The presence of the spirocyclic-1,3-ketolactam moiety would probably confer some interesting biological properties to products 3 , but the hemiaminal function also deserves to be considered for post-functionalizations. Thus, to start to illustrate the synthetic potential of platforms $\mathbf{3}$, some dehydration reactions were conducted in the presence of the Burgess reagent in refluxing THF for 2 hours (Scheme 5).

The corresponding enamides 6ia, $\mathbf{6 f d}$, and $\mathbf{6 g d}$, obtained from substrates 3ia, 3fd and 3gd, respectively, were isolated in good to excellent yields, as a 1:1 mixture of two diastereomers in the cases of $\mathbf{6 f d}$ and $\mathbf{6 g d}$.

Another interesting feature of the hemiaminal function in substrates $\mathbf{3}$ is the possibility to generate $\mathrm{N}$-acyliminium intermediates upon addition of Lewis acids, and subsequent trapping by an external nucleophile would result in substitution of the hydroxyl group. In order to translate this 


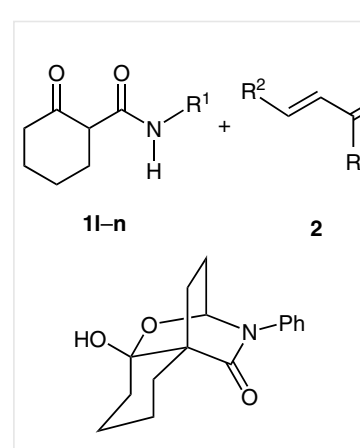

$51 a^{a}$

$70 \%(\mathrm{dr} 2: 1)$

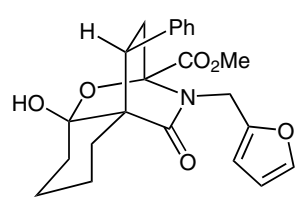

$5 \mathrm{md}^{\mathrm{c}}$

$64 \%(\mathrm{dr} 6: 1: 1)$
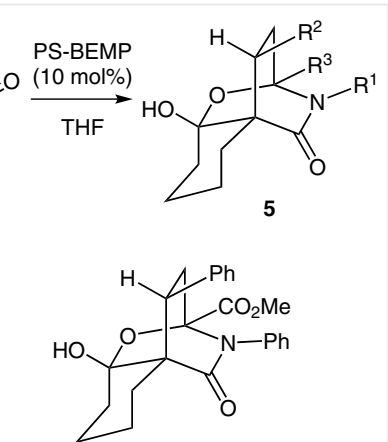

$51 d^{b}$

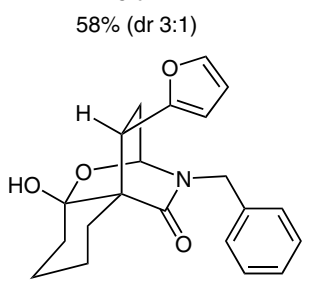

$5 \mathrm{nc}^{\mathrm{a}}$

$80 \%(\mathrm{dr} 10: 1)$
Scheme 4 Formation of polycyclic hemiacetals $\mathbf{5}$ from six-membered alicyclic 1,3-ketoamides $\mathbf{1 l}-\mathbf{n}$. ${ }^{\text {a }}$ Reaction conducted at $55^{\circ} \mathrm{C}$ for $24 \mathrm{~h}$.

${ }^{\mathrm{b}}$ Reaction conducted at r.t. for 30 h. ${ }^{\mathrm{c}}$ Reaction conducted at r.t. for $5 \mathrm{~h}$.

Table 2 Recyclability of the Supported Catalyst ${ }^{\mathrm{a}}$

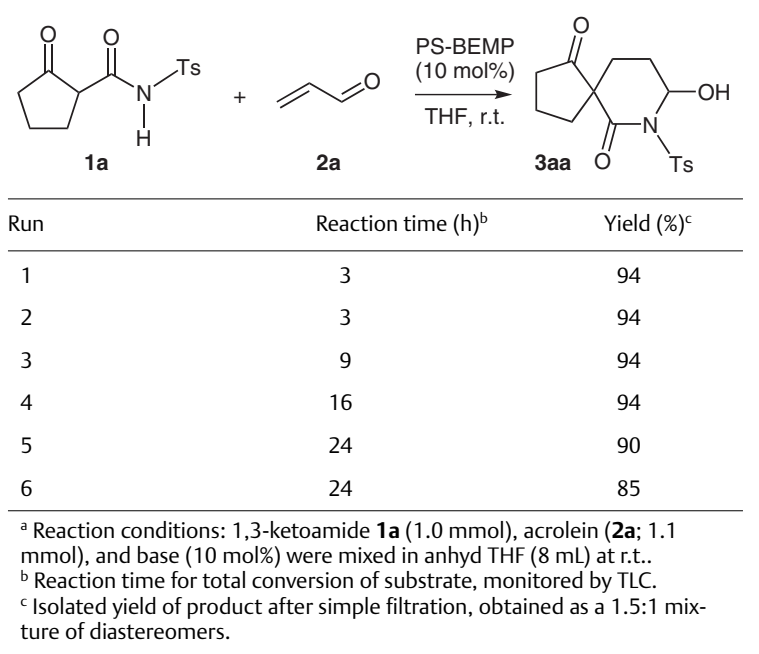

idea into practice, substrates $\mathbf{3}$ were reacted with various silylated nucleophiles $\mathbf{7 a - c}$ in the presence of $\mathrm{BF}_{3} \cdot \mathrm{OEt}_{2}$ at low temperature leading to functionalized products $\mathbf{8}$ in modest to excellent isolated yields, generally as a 1:1 mixture of two diastereomers (Scheme 6). Thus, the allyl moiety was successfully introduced in the structure of com-

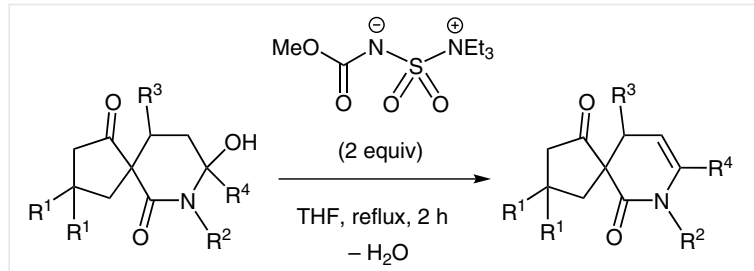

3<smiles>C=CCN1C=CCC2(CCCC2=O)C1=O</smiles>

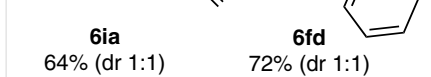<smiles>[120I-]</smiles>

6

Scheme 5 Dehydration of spirohemiaminals 3

pounds 8baa, 8caa, 8daa, 8iaa, and 8jaa through reaction with allyltrimethylsilane 7a. Trimethylsilyl cyanide (7b) also reacted with the iminium intermediate derived from substrate $\mathbf{3 f a}$, but the corresponding product $\mathbf{8 f a b}$ was isolated in a moderate yield. It is noteworthy that only one diastereomer could be isolated, and its structure has been determined by X-ray analysis of a single crystal (Figure 4). ${ }^{31}$ Similar results were obtained for the formation of product 8fac, containing a synthetically relevant allene moiety, which was obtained through reaction of $\mathbf{3 f a}$ with propargyl derivative $\mathbf{7 c}$, but in this case we were not able to ascertain the relative configuration.<smiles>N#C[C@@H]1CC[C@]2(CCCC2=O)C(=O)N1c1ccccc1</smiles>

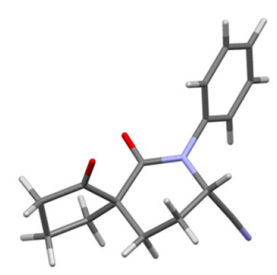

Figure 4 X-ray crystal structure analysis of spirolactam $8 \mathrm{fab}$

Finally, we identified compounds $\mathbf{8}$, bearing two allyl moieties, as potential substrates for ring closing-metathesis that could give access to original polycyclic spirolactams. Thus, treatment of spirolactams $\mathbf{8 b a a}$, 8iaa, and 8jaa with 5 mol\% of Grubbs second-generation catalyst resulted in the formation of the expected cyclized products 9baa, 9iaa, and 9jaa, respectively, which were isolated in good yields (Scheme 7).

In conclusion, we have shown that the commercially available, user-friendly polymer-supported organobase PSBEMP can be used as an efficient heterogeneous catalyst for 


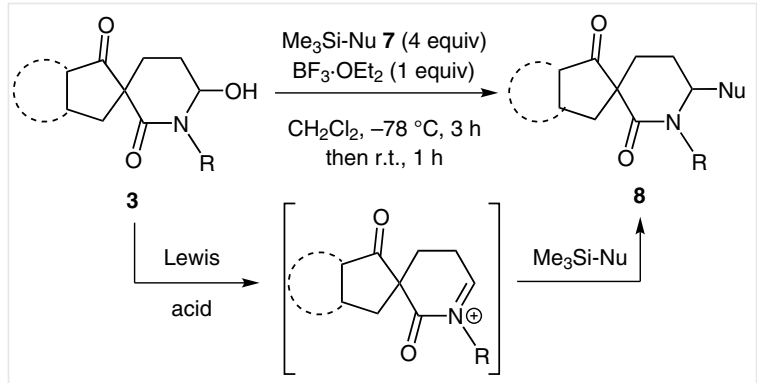

$\overbrace{\mathbf{7 a}}^{\mathrm{SiMe}_{3}} \stackrel{\mathrm{N} \equiv \mathrm{C}-\mathrm{SiMe}_{3}}{\mathbf{b}}=\overbrace{\mathbf{7 c}}^{\mathrm{SiMe}_{3}}$

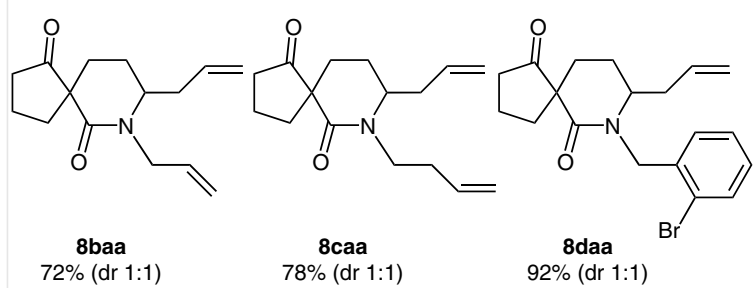<smiles>N#CC1CCC2(CCCC2=O)C(=O)N1c1ccccc1</smiles><smiles>C=CCC1CCC2(Cc3ccccc3C2=O)C(=O)N1CC=C</smiles>

8iaa
$87 \%(\mathrm{dr} 1: 1)$ 1

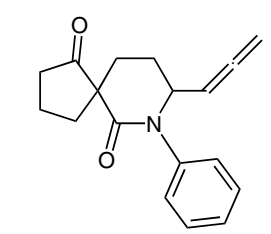

8 fac<smiles>C=CCC1CCC2(CCc3ccccc3C2=O)C(=O)N1CC=C</smiles>

8jaa

$60 \%($ dr $1: 1)$

Scheme 6 Functionalization of hemiaminals 3. a Only one diastereomer could be isolated.

domino reactions between cyclic 1,3-ketoamides and Michael acceptors for the direct access to spiro[n.5]-1,3-ketolactams. The products can be isolated by simple filtration in high yields and chemical purities, and the catalyst can be re-used without specific activation. From a sustainable chemistry point of view, this methodology advantageously completes the arsenal of reactions already described with phosphazenes in homogeneous catalysis, by limiting the number of isolation and purification steps. It has been also demonstrated that these spiroketolactams constitute interesting synthetic platforms, especially because of the presence of a hemiaminal moiety that serves as a precursor of

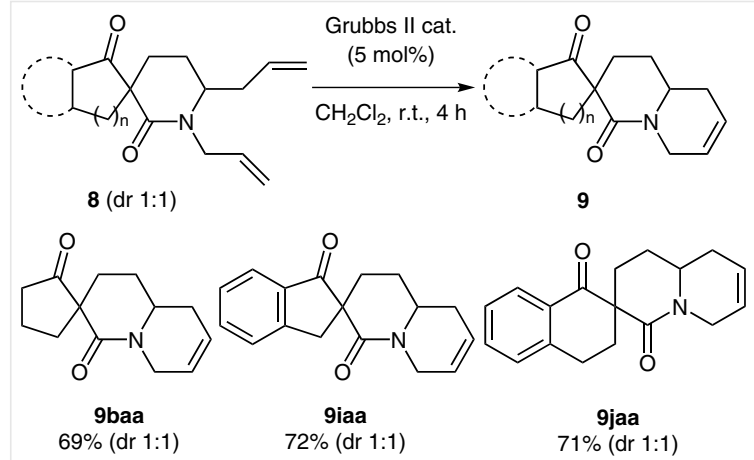

Scheme 7 Ring-closing-metathesis reactions on compounds 8

$\mathrm{N}$-acyliminium intermediates. Our findings set the stage for the future development of supported chiral phosphazene catalysts for domino reactions aimed at the enantioselective synthesis of heterocycles.

Unless otherwise specified, all commercially available reagents were used as received. Reactions were carried out under an argon atmosphere and all reagents were weighed and handled in air at r.t. Anhyd THF, toluene, and $\mathrm{CH}_{2} \mathrm{Cl}_{2}$ were obtained from a solvent purification system (MBRAUN). The reactions were monitored by TLC visualized by UV (254 nm) and/or with $p$-anisaldehyde and $\mathrm{H}_{2} \mathrm{SO}_{4}$ in EtOH. Flash chromatography was performed on 40-63 $\mu \mathrm{m}$ silica gel eluted with EtOAc/PE or $\mathrm{Et}_{2} \mathrm{O} / \mathrm{PE}$ (bp $40-60{ }^{\circ} \mathrm{C}$ ). NMR data were recorded at 300 or $400 \mathrm{MHz}$ (Bruker Avance spectrometers) in $\mathrm{CDCl}_{3}$ using as internal standards the residual $\mathrm{CHCl}_{3}$ signal for ${ }^{1} \mathrm{H}$ NMR $(\delta=7.26)$ and the deuterated solvent signal for ${ }^{13} \mathrm{C}$ NMR $(\delta=77.16)$. Coupling constants $(J)$ are in hertz $(\mathrm{Hz})$ and standard abbreviations are used to describe the signal multiplicities.

\section{Spiroheterocycles 3 and 4; General Procedure}

To a $10 \mathrm{~mL}$ one-necked round-bottomed flask, equipped with a magnetic stirring bar were added $\beta$-ketoamide 1 ( 1 equiv), freshly distilled THF $(0.5 \mathrm{M})$, Michael acceptor 2 (1.1 equiv), and PS-BEMP (10 mol\%). The mixture was stirred at r.t. or heated between 55 and $60{ }^{\circ} \mathrm{C}$ for the indicated time. After completion of the reaction (monitored by TLC), filtration, and concentration gave the resulting crude product, which was purified by chromatography on silica gel.

\section{8-Hydroxy-7-tosyl-7-azaspiro[4.5]decane-1,6-dione (3aa)}

Following the general procedure, the reaction of 4-methyl- $\mathrm{N}$-(2-oxocyclopentanecarbonyl)benzenesulfonamide (300 mg, $1.06 \mathrm{mmol})$ with acrolein (2a; $77.90 \mu \mathrm{L}, 1.16 \mathrm{mmol}$ ) in the presence of PS-BEMP ( $48.18 \mathrm{mg}, 10 \mathrm{~mol} \%)$ in THF $(2 \mathrm{~mL})$ at r.t. for $3 \mathrm{~h}$ afforded the product 3aa as a white solid ( $336.5 \mathrm{mg}, 94 \%$ ). The crude product was obtained with $1.5: 1 \mathrm{dr} ; R_{f}=0.48\left(\mathrm{Et}_{2} \mathrm{O} / \mathrm{PE}, 8: 2\right)$. Data given are for the mixture of two diastereomers.

${ }^{1} \mathrm{H} \mathrm{NMR}\left(400 \mathrm{MHz}, \mathrm{CDCl}_{3}\right): \delta=7.91-7.85(\mathrm{~m}, 4 \mathrm{H}), 7.31(\mathrm{~d}, J=8.1 \mathrm{~Hz}, 4$ $\mathrm{H}), 6.08-6.02(\mathrm{~m}, 2 \mathrm{H}), 4.18(\mathrm{dd}, J=6.0,0.9 \mathrm{~Hz}, 1 \mathrm{H}), 3.83(\mathrm{dd}, J=4.1$ $1.6 \mathrm{~Hz}, 1 \mathrm{H}), 2.67(\mathrm{~m}, 1 \mathrm{H}), 2.53-2.46(\mathrm{~m}, 1 \mathrm{H}), 2.42(\mathrm{~s}, 6 \mathrm{H}), 2.38-2.27$ (m, $5 \mathrm{H}), 2.24-2.03(\mathrm{~m}, 5 \mathrm{H}), 2.02-1.80(\mathrm{~m}, 8 \mathrm{H})$. 
${ }^{13} \mathrm{C}$ NMR $\left(75 \mathrm{MHz}, \mathrm{CDCl}_{3}\right): \delta=216.10$ (C), 214.73 (C), $171.12(\mathrm{C}$ ), 170.34 (C), 144.83 (C), 144.79 (C), 135.80 (C), 135.74 (C), 129.40 (4 $\mathrm{CH}), 128.53(2 \mathrm{CH}), 128.39(2 \mathrm{CH}), 79.19(\mathrm{CHOH}), 78.56(\mathrm{CHOH}), 58.71$ (C), $57.84(\mathrm{C}), 37.40\left(\mathrm{CH}_{2}\right), 36.49\left(\mathrm{CH}_{2}\right), 35.92\left(\mathrm{CH}_{2}\right), 27.80\left(\mathrm{CH}_{2}\right), 27.45$ $\left(\mathrm{CH}_{2}\right), 26.39\left(\mathrm{CH}_{2}\right), 24.42\left(\mathrm{CH}_{2}\right), 24.24\left(\mathrm{CH}_{2}\right), 21.65\left(\mathrm{CH}_{3}\right), 21.05,\left(\mathrm{CH}_{3}\right)$, $19.49\left(\mathrm{CH}_{2}\right), 19.3\left(\mathrm{CH}_{2}\right)$.

\section{7-Allyl-8-hydroxy-7-azaspiro[4.5]decane-1,6-dione (3ba)}

Following the general procedure, the reaction of $\mathrm{N}$-allyl-2-oxocyclopentanecarboxamide (122 mg, $0.73 \mathrm{mmol})$ with acrolein (2a; 53.64 $\mu \mathrm{L}, 0.803 \mathrm{mmol})$ in the presence of PS-BEMP $(33 \mathrm{mg}, 10 \mathrm{~mol} \%)$ in THF $(1.5 \mathrm{~mL})$ at r.t. for $24 \mathrm{~h}$ afforded the product $3 \mathbf{b a}$ as a colorless oil (142 $\mathrm{mg}, 87 \%)$. The crude product was obtained with $1: 1 \mathrm{dr} ; R_{f}=0.30$ $\left(\mathrm{Et}_{2} \mathrm{O}\right)$. Data given are for the mixture of two diastereomers.

${ }^{1} \mathrm{H} \mathrm{NMR}\left(300 \mathrm{MHz}, \mathrm{CDCl}_{3}\right): \delta=5.79-5.65(\mathrm{~m}, 2 \mathrm{H}), 5.21-5.06(\mathrm{~m}, 4 \mathrm{H})$, $4.93(\mathrm{dd}, J=5.5,3.1 \mathrm{~Hz}, 1 \mathrm{H}), 4.86(\mathrm{dd}, J=7.5,3.4 \mathrm{~Hz}, 1 \mathrm{H}), 4.64(\mathrm{~d}, J=$ $5.9 \mathrm{~Hz}, 1 \mathrm{H}), 4.50(\mathrm{~d}, J=8.1 \mathrm{~Hz}, 1 \mathrm{H}), 4.37-4.27(\mathrm{~m}, 2 \mathrm{H}), 3.67(\mathrm{dt}, J=$ $15.4,6.1 \mathrm{~Hz}, 2 \mathrm{H}), 2.73-2.63(\mathrm{~m}, 1 \mathrm{H}), 2.51-2.39(\mathrm{~m}, 3 \mathrm{H}), 2.34-2.21$ $(\mathrm{m}, 5 \mathrm{H}), 2.18-2.04(\mathrm{~m}, 3 \mathrm{H}), 1.91-1.79(\mathrm{~m}, 7 \mathrm{H}), 1.47(\mathrm{dt}, J=13.6,4.1$ $\mathrm{Hz}, 1 \mathrm{H})$.

${ }^{13} \mathrm{C} \operatorname{NMR}\left(75 \mathrm{MHz}, \mathrm{CDCl}_{3}\right): \delta=219.2$ (C), $216.2(\mathrm{C}), 170.6(\mathrm{C}), 170.2(\mathrm{C})$ 132.6 (CH), 132.5 (CH), $116.9\left(\mathrm{CH}_{2}\right), 116.3\left(\mathrm{CH}_{2}\right), 78.6(\mathrm{CH}), 78.3(\mathrm{CH})$ $56.6(\mathrm{C}), 55.8(\mathrm{C}), 46.7\left(\mathrm{CH}_{2}\right), 46.6\left(\mathrm{CH}_{2}\right), 38.9\left(\mathrm{CH}_{2}\right), 37.5\left(\mathrm{CH}_{2}\right), 36.1$ $\left(\mathrm{CH}_{2}\right), 35.9\left(\mathrm{CH}_{2}\right), 27.5\left(\mathrm{CH}_{2}\right), 26.4\left(\mathrm{CH}_{2}\right), 24.6\left(\mathrm{CH}_{2}\right), 24.4\left(\mathrm{CH}_{2}\right), 19.6$ $\left(\mathrm{CH}_{2}\right), 19.3\left(\mathrm{CH}_{2}\right)$.

HRMS (ESI+): $m / z[\mathrm{M}+\mathrm{H}]^{+}$calcd for $\mathrm{C}_{12} \mathrm{H}_{18} \mathrm{NO}_{3}$ : 224.1281; found: 224.1277.

\section{7-(But-3-en-1-yl)-8-hydroxy-7-azaspiro[4.5]decane-1,6-dione (3ca)}

Following the general procedure, the reaction of $\mathrm{N}$-(but-3-enyl)-2 oxocyclopentanecarboxamide ( $84 \mathrm{mg}, 0.46 \mathrm{mmol}$ ) with acrolein (2a; $33.80 \mu \mathrm{L}, 0.506 \mathrm{mmol}$ ) in the presence of PS-BEMP (21 mg, $10 \mathrm{~mol} \%)$ in THF $(1 \mathrm{~mL})$ at r.t. for $24 \mathrm{~h}$ afforded the product 3ca as a yellow oil (96 mg, 88\%). The crude product was obtained with $1: 1 \mathrm{dr} ; R_{f}=0.31$ $\left(\mathrm{Et}_{2} \mathrm{O}\right)$. Data given are for the mixture of two diastereomers.

${ }^{1} \mathrm{H} \mathrm{NMR}\left(300 \mathrm{MHz}, \mathrm{CDCl}_{3}\right): \delta=5.87-5.70(\mathrm{~m}, 2 \mathrm{H}), 5.15(\mathrm{~d}, J=1.6 \mathrm{~Hz}, 1$ H), $5.09-5.01(\mathrm{~m}, 4 \mathrm{H}), 4.90(\mathrm{t}, J=3.5 \mathrm{~Hz}, 1 \mathrm{H}), 3.63(\mathrm{dt}, J=14,7.2 \mathrm{~Hz}$ $2 \mathrm{H}), 3.33(\mathrm{td}, J=14.1,7.1 \mathrm{~Hz}, 2 \mathrm{H}), 2.79-2.71(\mathrm{~m}, 1 \mathrm{H}), 2.57-2.45(\mathrm{~m}, 2$ H), 2.39-2.16 (m, $10 \mathrm{H}), 1.96-1.75(\mathrm{~m}, 12 \mathrm{H}), 1.60-1.54(\mathrm{~m}, 1 \mathrm{H})$.

${ }^{13} \mathrm{C} \operatorname{NMR}\left(75 \mathrm{MHz}, \mathrm{CDCl}_{3}\right.$ ): $\delta=219.5$ (C), 216.7 (C), 170.6 (C), 170.3 (C), $135.4(2 \mathrm{CH}), 116.9\left(2 \mathrm{CH}_{2}\right), 80.1(\mathrm{CH}), 79.6(\mathrm{CH}), 56.7(\mathrm{C}), 55.8(\mathrm{C})$ $45.3\left(\mathrm{CH}_{2}\right), 44.6\left(\mathrm{CH}_{2}\right), 39.1\left(2 \mathrm{CH}_{2}\right), 37.8\left(\mathrm{CH}_{2}\right), 36.4\left(\mathrm{CH}_{2}\right), 36.2\left(\mathrm{CH}_{2}\right)$, 32.4 $\left(2 \mathrm{CH}_{2}\right), 27.7\left(\mathrm{CH}_{2}\right), 26.8\left(\mathrm{CH}_{2}\right), 24.9\left(\mathrm{CH}_{2}\right), 19.8\left(\mathrm{CH}_{2}\right), 19.5\left(\mathrm{CH}_{2}\right)$. HRMS (ESI+): $m / z[\mathrm{M}+\mathrm{H}]^{+}$calcd for $\mathrm{C}_{13} \mathrm{H}_{20} \mathrm{NO}_{3}: 238.1438$; found: 238.1441 .

\section{7-(2-Bromobenzyl)-9-hydroxy-7-azaspiro[4.5]decane-1,6-dione (3da)}

Following the general procedure, the reaction of $\mathrm{N}$-(2-bromobenzyl)2-oxocyclopentanecarboxamide (264 mg, $0.89 \mathrm{mmol}$ ) with acrolein $(65.40 \mu \mathrm{L}, 0.979 \mathrm{mmol})$ in the presence of PS-BEMP $(40.6 \mathrm{mg}, 10$ $\mathrm{mol} \%)$ in THF $(1.8 \mathrm{~mL})$ at r.t. for $24 \mathrm{~h}$ afforded the product 3da as a white solid (310 mg, 98\%). The crude product was obtained with 1:1 $\mathrm{dr} ; R_{f}=0.18\left(\mathrm{Et}_{2} \mathrm{O} / \mathrm{PE}, 7: 3\right)$. Data given are for the mixture of two diastereomers.
${ }^{1} \mathrm{H} \mathrm{NMR}\left(400 \mathrm{MHz}, \mathrm{CDCl}_{3}\right): \delta=7.53$ (ddd, $J=8.1,4.8,1.0 \mathrm{~Hz}, 2 \mathrm{H}$ ) 7.36-7.28 (m, $3 \mathrm{H}), 7.21$ (dd, $J=8,1.6 \mathrm{~Hz}, 1 \mathrm{H}), 7.14-7.09(\mathrm{~m}, 2 \mathrm{H})$, $5.12(\mathrm{~d}, J=16.3 \mathrm{~Hz}, 1 \mathrm{H}), 5.00(\mathrm{~d}, J=15.7 \mathrm{~Hz}, 1 \mathrm{H}), 4.95(\mathrm{~s}, 1 \mathrm{H}), 4.87(\mathrm{~s}$, $1 \mathrm{H}), 4.52(\mathrm{~d}, J=15.7 \mathrm{~Hz}, 1 \mathrm{H}), 4.44(\mathrm{~d}, J=16.3 \mathrm{~Hz}, 1 \mathrm{H}), 2.88-2.82(\mathrm{~m}$, $2 \mathrm{H}), 2.64-2.62(\mathrm{~m}, 1 \mathrm{H}), 2.57-2.48(\mathrm{~m}, 1 \mathrm{H}), 2.42-2.29(\mathrm{~m}, 5 \mathrm{H}), 2.23-$ $1.87(\mathrm{~m}, 10 \mathrm{H}), 1.81-1.75(\mathrm{~m}, 1 \mathrm{H}), 1.67-1.58(\mathrm{~m}, 2 \mathrm{H})$.

${ }^{13} \mathrm{C} \operatorname{NMR}\left(75 \mathrm{MHz}, \mathrm{CDCl}_{3}\right): \delta=217.8$ (C), 217.1 (C), 171.1 (C), 170.6 (C), $135.8(\mathrm{C}), 135.6(\mathrm{CH}), 132.5(\mathrm{CH}), 132.3(\mathrm{CH}), 128.54(\mathrm{CH}), 128.51$ $(\mathrm{CH}), 128.2(\mathrm{CH}), 127.67(\mathrm{CH}), 127.44(2 \mathrm{CH}), 123.03(\mathrm{C}), 122.7(\mathrm{C})$ $79.1(\mathrm{CH}), 79.0(\mathrm{CH}), 56.6(\mathrm{C}), 55.9(\mathrm{C}), 48.2\left(\mathrm{CH}_{2}\right), 48.0\left(\mathrm{CH}_{2}\right), 38.7$ $\left(\mathrm{CH}_{2}\right), 37.3\left(\mathrm{CH}_{2}\right), 36.0\left(\mathrm{CH}_{2}\right), 35.7\left(\mathrm{CH}_{2}\right), 27.5\left(\mathrm{CH}_{2}\right), 26.3\left(\mathrm{CH}_{2}\right), 25.4$ $\left(\mathrm{CH}_{2}\right), 24.5\left(\mathrm{CH}_{2}\right), 24.3\left(\mathrm{CH}_{2}\right), 19.4\left(\mathrm{CH}_{2}\right)$.

HRMS (ESI+): $m / z[\mathrm{M}+\mathrm{H}]^{+}$calcd for $\mathrm{C}_{16} \mathrm{H}_{19} \mathrm{BrNO}_{3}$ : 352.0543; found 352.0545 .

\section{7-(Furan-2-ylmethyl)-8-hydroxy-10-methyl-7-azaspiro[4.5]dec- ane-1,6-dione (3eb)}

Following the general procedure, the reaction of $\mathrm{N}$-(furan-2-ylmethyl)-2-oxocyclopentanecarboxamide $(264 \mathrm{mg}, 0.89 \mathrm{mmol}$ ) with crotonaldehyde (2b; $83.68 \mu \mathrm{L}, 0.979 \mathrm{mmol})$ in the presence of PS-BEMP ( $40.6 \mathrm{mg}, 10 \mathrm{~mol} \%$ ) in THF $(1.8 \mathrm{~mL}$ ) at r.t. for $24 \mathrm{~h}$ afforded the product 3eb as a white solid (310 mg, 98\%). The crude product was obtained with 6:4:1:1 dr. Two diastereomers were separated (silica gel chromatography).

First fraction: one diastereomer was isolated as a yellow solid $(43 \mathrm{mg}$ $12 \%) ; R_{f}=0.7\left(\mathrm{Et}_{2} \mathrm{O} / \mathrm{PE}, 9: 1\right)$.

${ }^{1} \mathrm{H} \mathrm{NMR}\left(400 \mathrm{MHz}, \mathrm{CDCl}_{3}\right): \delta=7.33(\mathrm{dd}, J=1.8,0.7 \mathrm{~Hz}, 1 \mathrm{H}), 6.30(\mathrm{dd}$, $J=3.2,1.9 \mathrm{~Hz}, 1 \mathrm{H}), 6.24(\mathrm{~d}, J=3.2 \mathrm{~Hz}, 1 \mathrm{H}), 5.30(\mathrm{~s}, 1 \mathrm{H}), 5.02(\mathrm{~d}, J=$ $15.3 \mathrm{~Hz}, 1 \mathrm{H}), 4.91(\mathrm{t}, J=12.7 \mathrm{~Hz}, 1 \mathrm{H}), 4.39(\mathrm{~d}, J=15.3 \mathrm{~Hz}, 1 \mathrm{H}), 3.37$ $(\mathrm{d}, J=12.2 \mathrm{~Hz}, 1 \mathrm{H}), 2.92(\mathrm{t}, J=7.8 \mathrm{~Hz}, 1 \mathrm{H}), 2.47-2.42(\mathrm{~m}, 1 \mathrm{H}), 2.24-$ 2.20 (m, 2 H), 2.12-2.04 (m, 1 H), 2.07-1.94 (m, 3 H), 0.98 (d, $J=7.1$ $\mathrm{Hz}, 3 \mathrm{H})$.

${ }^{13} \mathrm{C}$ NMR $\left(75 \mathrm{MHz}, \mathrm{CDCl}_{3}\right): \delta=219.15(\mathrm{C}), 169.61(\mathrm{C}), 151.17(\mathrm{C})$, 142.22 (CH), $110.44(\mathrm{CH}), 108.57(\mathrm{CH}), 78.77(\mathrm{CHOH}), 60.23(\mathrm{C}), 40.97$ $\left(\mathrm{CH}_{2}\right), 39.64\left(\mathrm{CH}_{2}\right), 36.62\left(\mathrm{CH}_{2}\right), 34.35(\mathrm{CH}), 33.29\left(\mathrm{CH}_{2}\right), 20.76\left(\mathrm{CH}_{2}\right)$, $17.19\left(\mathrm{CH}_{3}\right)$.

Second fraction: incomplete separation; two diastereomers.

${ }^{1} \mathrm{H}$ NMR $\left(400 \mathrm{MHz}, \mathrm{CDCl}_{3}\right): \delta=7.35(\mathrm{~s}, 1 \mathrm{H}), 7.33(\mathrm{~s}, 1 \mathrm{H}), 6.33(\mathrm{~d}, J=$ $3.0 \mathrm{~Hz}, 1 \mathrm{H}), 6.31(\mathrm{~d}, J=2.1 \mathrm{~Hz}, 2 \mathrm{H}), 6.27(\mathrm{~d}, J=3.0 \mathrm{~Hz}, 1 \mathrm{H}), 5.11(\mathrm{~s}, 1$ H), $4.94(\mathrm{~s}, 1 \mathrm{H}), 4.89(\mathrm{~d}, J=15.4 \mathrm{~Hz}, 1 \mathrm{H}), 4.67(\mathrm{~d}, J=15.4 \mathrm{~Hz}, 1 \mathrm{H})$, $4.53(\mathrm{~d}, J=15.6 \mathrm{~Hz}, 1 \mathrm{H}), 4.32(\mathrm{~d}, J=15.4 \mathrm{~Hz}, 1 \mathrm{H}), 3.28-3.22(\mathrm{~m}, 1 \mathrm{H})$, 2.69-1.62 (m, $19 \mathrm{H}), 0.98(\mathrm{~d}, J=6.7 \mathrm{~Hz}, 2 \mathrm{H}), 0.85(\mathrm{~d}, J=6.9 \mathrm{~Hz}, 4 \mathrm{H})$.

${ }^{13} \mathrm{C} \operatorname{NMR}\left(75 \mathrm{MHz}, \mathrm{CDCl}_{3}\right): \delta=218.9$ (C), 217.5 (C), 171.9 (C), 170.8 (C), $150.4(2 \mathrm{C}), 142.1(2 \mathrm{CH}), 110.4(\mathrm{CH}), 110.3(\mathrm{CH}), 108.4(\mathrm{CH}), 107.3$ $(\mathrm{CH}), 78.7(\mathrm{CH}), 77.8(\mathrm{CH}), 60.2(\mathrm{C}), 59.2(\mathrm{C}), 41.8\left(\mathrm{CH}_{2}\right), 40.4\left(\mathrm{CH}_{2}\right)$, $39.87\left(\mathrm{CH}_{2}\right), 39.81\left(\mathrm{CH}_{2}\right), 35.3\left(\mathrm{CH}_{2}\right), 34.5\left(\mathrm{CH}_{2}\right), 34.2\left(\mathrm{CH}_{2}\right), 30.9(\mathrm{CH})$, $29.8\left(\mathrm{CH}_{2}\right), 27.4(\mathrm{CH}), 20.7\left(\mathrm{CH}_{2}\right), 19.9\left(\mathrm{CH}_{2}\right), 16.2\left(\mathrm{CH}_{3}\right), 15.7\left(\mathrm{CH}_{3}\right)$.

Third fraction: one diastereomer was isolated as a yellow solid (30 $\mathrm{mg}, 10 \%) ; R_{f}=0.39\left(\mathrm{Et}_{2} \mathrm{O} / \mathrm{PE}, 9: 1\right)$

${ }^{1} \mathrm{H} \mathrm{NMR}\left(300 \mathrm{MHz}, \mathrm{CDCl}_{3}\right): \delta=7.26(\mathrm{t}, J=3.8 \mathrm{~Hz}, 1 \mathrm{H}), 6.25-6.21(\mathrm{~m}, 1$ H), $6.19(\mathrm{~d}, J=3.0 \mathrm{~Hz}, 1 \mathrm{H}), 4.90-4.81(\mathrm{~m}, 1 \mathrm{H}), 4.77(\mathrm{~d}, J=15.4 \mathrm{~Hz}, 1$ H), $4.37(\mathrm{~d}, J=15.4 \mathrm{~Hz}, 1 \mathrm{H}), 3.38(\mathrm{dd}, J=14.9,7.8 \mathrm{~Hz}, 1 \mathrm{H}), 2.53(\mathrm{~m}, 1$ $\mathrm{H}), 2.11(\mathrm{~m}, 5 \mathrm{H}), 1.79$ (ddd, $J=8.3,6.9,3.8 \mathrm{~Hz}, 1 \mathrm{H}), 1.51(\mathrm{~m} 1 \mathrm{H}), 1.18$ $(\mathrm{s}, 1 \mathrm{H}), 0.77(\mathrm{~d}, J=6.8 \mathrm{~Hz}, 3 \mathrm{H})$.

${ }^{13} \mathrm{C}$ NMR $\left(75 \mathrm{MHz}, \mathrm{CDCl}_{3}\right): \delta=217.86(\mathrm{C}), 172.25$ (C), 150.79 (C), $142.15(\mathrm{CH}), 110.72(\mathrm{CH}), 108.69(\mathrm{CH}), 79.42(\mathrm{CHOH}), 59.78(\mathrm{C}), 39.91$ $\left(\mathrm{CH}_{2}\right), 38.55\left(\mathrm{CH}_{2}\right), 36.51\left(\mathrm{CH}_{2}\right), 29.44\left(\mathrm{CH}_{2}\right), 29.34(\mathrm{CH}), 19.95\left(\mathrm{CH}_{2}\right)$, $16.03\left(\mathrm{CH}_{3}\right)$ 


\section{0-(Furan-2-yl)-7-(furan-2-ylmethyl)-8-hydroxy-7-aza- spiro[4.5]decane-1,6-dione (3ec)}

Following the general procedure, the reaction of $\mathrm{N}$-(furan-2-ylmethyl)-2-oxocyclopentanecarboxamide ( $264 \mathrm{mg}, 0.89 \mathrm{mmol}$ ) with furylacrolein $(\mathbf{2 c} ; 70 \mathrm{~mL}, 0.979 \mathrm{mmol}$ ) in the presence of PS-BEMP (40.6 $\mathrm{mg}, 10 \mathrm{~mol} \%)$ in THF $(1.8 \mathrm{~mL})$ at r.t. for $24 \mathrm{~h}$ afforded the product $3 e c$ as a white solid (310 mg, 98\%). The crude product was obtained with $6: 4: 1: 1 \mathrm{dr} ; R_{f}=0.18\left(\mathrm{Et}_{2} \mathrm{O} / \mathrm{PE}, 7: 3\right)$. Data given are for the mixture of four diastereomers.

${ }^{1} \mathrm{H} \mathrm{NMR}\left(300 \mathrm{MHz}, \mathrm{CDCl}_{3}\right): \delta=7.28(\mathrm{~s}, 1 \mathrm{H}), 7.26(\mathrm{dd}, J=1.6,0.8 \mathrm{~Hz}, 3$ $\mathrm{H}), 7.24(\mathrm{dd}, J=1.9,0.6 \mathrm{~Hz}, 1 \mathrm{H}), 7.23(\mathrm{~d}, J=1.3 \mathrm{~Hz}, 1 \mathrm{H}), 7.22-7.20(\mathrm{~m}$, $2 \mathrm{H}), 6.26-6.22(\mathrm{~m}, 8 \mathrm{H}), 6.22-6.19(\mathrm{~m}, 4 \mathrm{H}), 6.02(\mathrm{~d}, J=3.3 \mathrm{~Hz}, 1 \mathrm{H})$, $6.00(\mathrm{~d}, J=3.3 \mathrm{~Hz}, 1 \mathrm{H}), 5.97(\mathrm{~d}, J=3.2 \mathrm{~Hz}, 2 \mathrm{H}), 5.16-5.10(\mathrm{~m}, 1 \mathrm{H})$, 5.09-5.02 (m, $2 \mathrm{H}), 4.95$ (d, J = $15.5 \mathrm{~Hz}, 4 \mathrm{H}), 4.90(\mathrm{~d}, J=2.8 \mathrm{~Hz}, 1 \mathrm{H})$, $4.84(\mathrm{~d}, J=6.1 \mathrm{~Hz}, 3 \mathrm{H}), 4.81(\mathrm{~d}, J=4.8 \mathrm{~Hz}, 1 \mathrm{H}), 4.45-4.37(\mathrm{~m}, 1 \mathrm{H})$ $4.33(\mathrm{~d}, J=15.8 \mathrm{~Hz}, 1 \mathrm{H}), 4.19(\mathrm{~d}, J=15.5 \mathrm{~Hz}, 2 \mathrm{H}), 4.08(\mathrm{~d}, J=7.2 \mathrm{~Hz}, 1$ $\mathrm{H}), 4.05(\mathrm{~d}, J=2.6 \mathrm{~Hz}, 1 \mathrm{H}), 4.04-4.01(\mathrm{~m}, 1 \mathrm{H}), 3.67(\mathrm{dd}, J=13.7,2.7$ $\mathrm{Hz}, 1 \mathrm{H}), 3.50$ (dd, $J=13.5,2.4 \mathrm{~Hz}, 1 \mathrm{H}), 3.06$ (dd, $J=13.9,4.4 \mathrm{~Hz}, 1 \mathrm{H}$ ), 2.94 (dd, $J=13.8,3.4 \mathrm{~Hz}, 1 \mathrm{H}), 2.85(\mathrm{~d}, J=13.1 \mathrm{~Hz}, 1 \mathrm{H}), 2.79-2.70(\mathrm{~m}$, $1 \mathrm{H}), 2.49-2.38$ (m, $3 \mathrm{H}), 2.23-2.03$ (m, $14 \mathrm{H}), 1.98-1.81$ (m, $10 \mathrm{H})$.

${ }^{13} \mathrm{C} \mathrm{NMR}\left(75 \mathrm{MHz}, \mathrm{CDCl}_{3}\right.$ ): $\delta$ (major 1 ) = 218.4(C), $171.3(\mathrm{C}), 153.2(\mathrm{C})$, $150.2(\mathrm{C}), 142.2(2 \mathrm{CH}), 110.3(2 \mathrm{CH}), 108.6(\mathrm{CH}), 106.9(\mathrm{CH}), 77.3(\mathrm{C})$, $59.8(\mathrm{C}), 40.1\left(\mathrm{CH}_{2}\right), 39.4\left(\mathrm{CH}_{2}\right), 39.3\left(\mathrm{CH}_{2}\right), 32.9(\mathrm{CH}), 31.5\left(\mathrm{CH}_{2}\right)$, $19.30\left(\mathrm{CH}_{2}\right) ; \delta$ (major 2) = 216.8 (C), $170.1(\mathrm{C}), 153.6(\mathrm{C}), 150.4(\mathrm{C})$ $142.0(2 \mathrm{CH}), 141.6(\mathrm{CH}), 141.3(\mathrm{CH}), 110.4(\mathrm{CH}), 107.8(\mathrm{CH}), 78.3(\mathrm{C})$, $58.8(\mathrm{C}), 41.9\left(\mathrm{CH}_{2}\right), 36.5(\mathrm{CH}), 34.1\left(\mathrm{CH}_{2}\right), 31.7\left(\mathrm{CH}_{2}\right), 30.9\left(\mathrm{CH}_{2}\right), 19.7$ $\left(\mathrm{CH}_{2}\right)$; $\delta($ minor $1+$ minor 2$)=218.2(\mathrm{C}), 217.5(\mathrm{C}), 171.5(\mathrm{C}), 171.4$ (C), $152.6(\mathrm{C}), 152.5(\mathrm{C}), 150.5(\mathrm{C}), 150.4(\mathrm{C}), 142.1(\mathrm{CH}), 141.8(\mathrm{CH})$, 141.7 (CH), $110.6(\mathrm{CH}), 110.5(\mathrm{CH}), 108.5(\mathrm{CH}), 108.1(\mathrm{CH}), 107.4(\mathrm{CH})$, $107.3(\mathrm{CH}), 107.28(\mathrm{CH}), 107.20(\mathrm{CH}), 110.2(\mathrm{CH}), 78.6(\mathrm{C}), 78.3(\mathrm{C})$, $60.4(\mathrm{C}), 59.3(\mathrm{C}), 39.7\left(\mathrm{CH}_{2}\right), 39.5\left(\mathrm{CH}_{2}\right), 39.2\left(\mathrm{CH}_{2}\right), 37.86\left(\mathrm{CH}_{2}\right), 36.0$ $\left(\mathrm{CH}_{2}\right), 35.9\left(\mathrm{CH}_{2}\right), 34.5(\mathrm{CH}), 33.1\left(\mathrm{CH}_{2}\right), 33.1\left(\mathrm{CH}_{2}\right), 31.0\left(\mathrm{CH}_{2}\right), 20.9$ (CH), $19.36\left(\mathrm{CH}_{2}\right)$

\section{Methyl 7-(Furan-2-ylmethyl)-8-hydroxy-1,6-dioxo-10-phenyl-7-} azaspiro[4.5]decane-8-carboxylate (3ed)

Following the general procedure, the reaction of $N$-(furan-2-ylmethyl)-2-oxocyclopentanecarboxamide ( $200 \mathrm{mg}, 0.96 \mathrm{mmol}$ ) with methyl (E)-2-oxo-4-phenylbut-3-enoate (2d; $201 \mathrm{mg}, 1.05 \mathrm{mmol})$ in the presence of PS-BEMP (44 mg, $10 \mathrm{~mol} \%$ ) in THF (2 mL) at r.t. for $5 \mathrm{~h}$ afforded the product 3ed as a white solid (368 $\mathrm{mg}, 98 \%$ ). The crude product was obtained with 5:1.5:1:1 dr. One diastereiosomer was separated (silica gel chromatography).

First fraction: one diastereomer was isolated as a yellow solid (44 mg, $12 \%) ; \mathrm{mp} 165^{\circ} \mathrm{C} ; R_{f}=0.56\left(\mathrm{Et}_{2} \mathrm{O} / \mathrm{PE}, 7: 3\right)$.

${ }^{1} \mathrm{H}$ NMR $\left(300 \mathrm{MHz}, \mathrm{CDCl}_{3}\right): \delta=7.23(\mathrm{~d}, J=0.9 \mathrm{~Hz}, 1 \mathrm{H}), 7.19(\mathrm{t}, J=5.2$ $\mathrm{Hz}, 2 \mathrm{H}), 7.14-7.06(\mathrm{~m}, 2 \mathrm{H}), 6.22$ (dd, $J=3.1,1.9 \mathrm{~Hz}, 1 \mathrm{H}), 6.16$ (d, $J=$ $3.2 \mathrm{~Hz}, 1 \mathrm{H}), 4.38$ (s, $2 \mathrm{H}), 3.69(\mathrm{~s}, 3 \mathrm{H}), 3.64-3.53(\mathrm{~m}, 1 \mathrm{H}), 3.43$ (dd, $J=$ 13.8, $2.1 \mathrm{~Hz}, 1 \mathrm{H}), 2.79-2.69$ (m, $1 \mathrm{H}), 2.20-1.98$ (m, $2 \mathrm{H}), 1.92-1.81$ $(\mathrm{m}, 1 \mathrm{H}), 1.77(\mathrm{dd}, J=12.9,2.2 \mathrm{~Hz}, 1 \mathrm{H}), 1.68-1.55(\mathrm{~m}, 1 \mathrm{H}), 1.10(\mathrm{~d}, J=$ $14.8 \mathrm{~Hz}, 1 \mathrm{H}), 0.87-0.76(\mathrm{~m}, 1 \mathrm{H})$.

${ }^{13} \mathrm{C}$ NMR (75 MHz, $\mathrm{CDCl}_{3}$ ): $\delta=217.34(\mathrm{C}), 172.76$ (C), 171.01 (C), $150.73(\mathrm{C}), 141.87(\mathrm{CH}), 139.16(\mathrm{C}), 128.93(2 \mathrm{CH}), 128.89(2 \mathrm{CH})$, $127.92(\mathrm{CH}), 110.68(\mathrm{CH}), 108.76(\mathrm{CH}), 85.60(\mathrm{COH}), 60.63(\mathrm{C}), 54.39$ $\left(\mathrm{CH}_{3}\right), 43.47(\mathrm{CH}), 40.75\left(\mathrm{CH}_{2}\right), 40.48\left(\mathrm{CH}_{2}\right), 36.02\left(\mathrm{CH}_{2}\right), 34.26\left(\mathrm{CH}_{2}\right)$, $20.18\left(\mathrm{CH}_{2}\right)$.

Second fraction: a mixture of two diastereomers was isolated (295 $\mathrm{mg}, 80 \%)$ as a yellow oil; $R_{\mathrm{f}}=0.44\left(\mathrm{Et}_{2} \mathrm{O} / \mathrm{PE}, 7: 3\right)$. The major diastereomer partially crystallized as white needles for X-ray analysis.
${ }^{1} \mathrm{H}$ NMR $\left(300 \mathrm{MHz}, \mathrm{CDCl}_{3}\right): \delta=7.41-7.26(\mathrm{~m}, 8 \mathrm{H}), 7.25-7.03(\mathrm{~m}, 5 \mathrm{H})$, $6.31(\mathrm{~d}, J=1.8 \mathrm{~Hz}, 1 \mathrm{H}), 6.29(\mathrm{~d}, J=1.9 \mathrm{~Hz}, 1 \mathrm{H}), 6.28(\mathrm{~d}, J=3.4 \mathrm{~Hz}, 1 \mathrm{H})$, $6.25(\mathrm{~d}, J=3.2 \mathrm{~Hz}, 1 \mathrm{H}), 4.80(\mathrm{~d}, J=15.5 \mathrm{~Hz}, 1 \mathrm{H}), 4.67(\mathrm{~s}, 1 \mathrm{H}), 4.63(\mathrm{~d}$, $J=2.8 \mathrm{~Hz}, 1 \mathrm{H}), 4.58(\mathrm{~d}, J=3.8 \mathrm{~Hz}, 2 \mathrm{H}), 4.31(\mathrm{~d}, J=15.9 \mathrm{~Hz}, 1 \mathrm{H}), 4.16$ (dd, $J=13.9,2.2 \mathrm{~Hz}, 1 \mathrm{H}), 3.71(\mathrm{~s}, 1 \mathrm{H}), 3.67(\mathrm{~s}, 3 \mathrm{H}), 3.47-3.24(\mathrm{~m}, 1$ $\mathrm{H}), 2.91(\mathrm{dd}, J=13.9,6.7 \mathrm{~Hz}, 1 \mathrm{H}), 2.75(\mathrm{t}, J=13.6 \mathrm{~Hz}, 2 \mathrm{H}), 2.45-2.07$ (m, $8 \mathrm{H}), 2.04-1.67(\mathrm{~m}, 4 \mathrm{H}), 1.11-0.95(\mathrm{~m}, 2 \mathrm{H})$.

${ }^{13} \mathrm{C} \mathrm{NMR}\left(75 \mathrm{MHz}, \mathrm{CDCl}_{3}\right.$ ): $\delta$ (major) = $217.1(\mathrm{C}), 172.7(\mathrm{C}), 172.3(\mathrm{C})$, $150.5(\mathrm{C}), 141.8(\mathrm{CH}), 138.1(\mathrm{C}), 128.8(\mathrm{CH}), 128.5(2 \mathrm{CH}), 127.4(2$ $\mathrm{CH}), 110.5(\mathrm{CH}), 108.8(\mathrm{CH}), 84.8(\mathrm{COH}), 61.4(\mathrm{C}), 54.1\left(\mathrm{CH}_{3}\right), 40.23$ $(\mathrm{CH}), 39.8\left(\mathrm{CH}_{2}\right), 38.0\left(\mathrm{CH}_{2}\right), 36.1\left(\mathrm{CH}_{2}\right), 30.1\left(\mathrm{CH}_{2}\right), 19.5\left(\mathrm{CH}_{2}\right) ; \delta(\mathrm{mi}-$ nor $)=217.6(\mathrm{C}), 172.1(\mathrm{C}), 170.2(\mathrm{C}), 150.8(\mathrm{C}), 141.7(\mathrm{CH}), 138.6(\mathrm{C})$, $128.6(2 \mathrm{CH}), 128.2(\mathrm{CH}), 127.9(2 \mathrm{CH}), 110.4(\mathrm{CH}), 109.0(\mathrm{CH}), 84.9$ $(\mathrm{COH}), 59.9(\mathrm{C}), 53.8\left(\mathrm{CH}_{3}\right), 49.9(\mathrm{CH}), 40.2\left(\mathrm{CH}_{2}\right), 38.1\left(\mathrm{CH}_{2}\right), 37.0$ $\left(\mathrm{CH}_{2}\right), 33.6\left(\mathrm{CH}_{2}\right), 20.0\left(\mathrm{CH}_{2}\right)$.

HRMS (ESI+): $m / z[\mathrm{M}+\mathrm{H}]^{+}$calcd for $\mathrm{C}_{22} \mathrm{H}_{20} \mathrm{NO}_{6}: 398.1598$; found: 398.1597.

\section{Methyl 8-Hydroxy-1,6-dioxo-7,10-diphenyl-7-azaspiro[4.5]dec- ane-8-carboxylate (3fd)}

Following the general procedure, the reaction of 2-oxo- $N$-phenylcyclopentanecarboxamide ( $500 \mathrm{mg}, 2.46 \mathrm{mmol}$ ) with methyl $(E)-2$-oxo4-phenylbut-3-enoate (2d; $514 \mathrm{mg}, 2.70 \mathrm{mmol})$ in the presence of PSBEMP (112.5 mg, $10 \mathrm{~mol} \%$ ) in THF ( $5 \mathrm{~mL}$ ) at r.t. for $32 \mathrm{~h}$ afforded the product $\mathbf{3 f d}$ as a white solid $(96.5 \mathrm{mg}, 98 \%)$. The crude product was obtained with 6:2:2:1 dr. Two diastereomers were separated.

First fraction: one diastereomer was isolated as a yellow oil (155 mg, $16 \%) ; R_{f}=0.75\left(\mathrm{Et}_{2} \mathrm{O} / \mathrm{PE}, 1: 1\right)$.

${ }^{1} \mathrm{H}$ NMR $\left(300 \mathrm{MHz}, \mathrm{CDCl}_{3}\right): \delta=7.37-7.28(\mathrm{~m}, 6 \mathrm{H}), 7.23-7.18(\mathrm{~m}, 4 \mathrm{H})$, $5.27(\mathrm{~s}, 1 \mathrm{H}, \mathrm{OH}), 4.56$ (d, $J=2.2 \mathrm{~Hz}, 1 \mathrm{H}), 4.39$ (dd, $J=13.7,2 \mathrm{~Hz}, 1 \mathrm{H})$, $3.71(\mathrm{~s}, 3 \mathrm{H}), 2.96(\mathrm{t}, J=13.6 \mathrm{~Hz}, 1 \mathrm{H}), 2.39-2.33(\mathrm{~m}, 2 \mathrm{H}), 2.31-2.24$ (m, $1 \mathrm{H}), 2.21(\mathrm{dd}, J=13,2.2 \mathrm{~Hz}, 1 \mathrm{H}), 1.94-1.77(\mathrm{~m}, 2 \mathrm{H})$.

${ }^{13} \mathrm{C}$ NMR (75 MHz, $\mathrm{CDCl}_{3}$ ): $\delta=217.02(\mathrm{C}), 172.21$ (C), $170.85(\mathrm{C})$, $139.22(\mathrm{C}), 138.51(\mathrm{C}), 129.28(2 \mathrm{CH}), 129.08(2 \mathrm{CH}), 129.04(2 \mathrm{CH})$, $128.86(2 \mathrm{CH}), 128.48(\mathrm{CH}), 127.89(\mathrm{CH}), 86.16(\mathrm{C}), 60.61(\mathrm{C}), 53.63$ $\left(\mathrm{CH}_{3}\right), 43.87(\mathrm{CH}), 39.98(\mathrm{CH}), 35.81\left(\mathrm{CH}_{2}\right), 34.03\left(\mathrm{CH}_{2}\right), 19.78\left(\mathrm{CH}_{2}\right)$.

Second fraction: one diastereomer was isolated as a yellow oil (464 $\mathrm{mg}, 48 \%) ; R_{\mathrm{f}}=0.48\left(\mathrm{Et}_{2} \mathrm{O} / \mathrm{PE}, 1: 1\right)$.

${ }^{1} \mathrm{H}$ NMR $\left(300 \mathrm{MHz}, \mathrm{CDCl}_{3}\right): \delta=7.36-7.32(\mathrm{~m}, 6 \mathrm{H}), 7.24-7.19(\mathrm{~m}, 5 \mathrm{H})$, $4.62(\mathrm{~s}, 1 \mathrm{H}), 4.40(\mathrm{dd}, J=13.8,2.2 \mathrm{~Hz}, 1 \mathrm{H}), 3.70(\mathrm{~s}, 3 \mathrm{H}), 2.97(\mathrm{t}, J=$ $13.5 \mathrm{~Hz}, 1 \mathrm{H}), 2.40-2.33(\mathrm{~m}, 2 \mathrm{H}), 2.30$ (dd, $J=13.2,6.7 \mathrm{~Hz}, 1 \mathrm{H}), 2.20$ (dd, $J=13.1,2.4 \mathrm{~Hz}, 1 \mathrm{H}), 1.94-1.77(\mathrm{~m}, 2 \mathrm{H})$.

${ }^{13} \mathrm{C} \mathrm{NMR}\left(75 \mathrm{MHz}, \mathrm{CDCl}_{3}\right.$ ): $\delta=216.7$ (C), 172.7 (C), $171.0(\mathrm{C}), 138.2(\mathrm{C})$, $137.1(\mathrm{C}), 129.3(2 \mathrm{CH}), 128.8(2 \mathrm{CH}), 128.6(\mathrm{CH}), 128.5(\mathrm{CH}), 128.3(2$ $\mathrm{CH}), 127.2(2 \mathrm{CH}), 86.1(\mathrm{C}), 61.4(\mathrm{C}), 53.5\left(\mathrm{CH}_{3}\right), 39.6\left(\mathrm{CH}_{2}\right), 38.0(\mathrm{CH})$, $35.5\left(\mathrm{CH}_{2}\right), 30.3\left(\mathrm{CH}_{2}\right), 19.3\left(\mathrm{CH}_{2}\right)$.

HRMS (ESI+): $m / z[\mathrm{M}+\mathrm{H}]^{+}$calcd for $\mathrm{C}_{23} \mathrm{H}_{24} \mathrm{NO}_{5}$ : 394.1649; found: 394.1661 .

Methyl 8-Hydroxy-3,3-dimethyl-1,6-dioxo-10-phenyl-7-azaspiro[4.5]decane-8-carboxylate (3gd)

Following the general procedure, the reaction of 4,4-dimethyl-2-oxocyclopentanecarboxamide (170 mg, $1.09 \mathrm{mmol}$ ) with methyl $(E)-2$ oxo-4-phenylbut-3-enoate (2d; $229.69 \mathrm{mg}, 1.20 \mathrm{mmol})$ in the presence of PS-BEMP (49 mg, $10 \mathrm{~mol} \%$ ) in THF (2.5 mL) at r.t. for $24 \mathrm{~h}$ afforded the product $3 g d$ as a white solid $(160 \mathrm{mg}, 42 \%)$. The crude product was obtained with 2:2:1:1 dr; $R_{f}=0.35\left(\mathrm{Et}_{2} \mathrm{O}\right)$. Data given are for the mixture of four diastereomers. 
${ }^{1} \mathrm{H}$ NMR $\left(300 \mathrm{MHz}, \mathrm{CDCl}_{3}\right): \delta$ (characteristic peaks) $=3.87(\mathrm{~s}, 2 \mathrm{H})$, $3.85(\mathrm{~s}, 3 \mathrm{H}), 3.83(\mathrm{~s}, 1 \mathrm{H}), 3.81(\mathrm{~s}, 1 \mathrm{H})$.

${ }^{13} \mathrm{C}$ NMR $\left(75 \mathrm{MHz}, \mathrm{CDCl}_{3}\right.$ ): $\delta$ (major 1$)=216.9(\mathrm{C}), 182.6(\mathrm{C}), 174.87$ (C), $138.8(\mathrm{C}), 129.29(2 \mathrm{CH}), 129.27(2 \mathrm{CH}), 128.8(\mathrm{CH}), 81.2(\mathrm{C}), 68.0$ $\left(\mathrm{CH}_{3}\right), 63.4(\mathrm{C}), 45.4\left(\mathrm{CH}_{2}\right), 43.3(\mathrm{CH}), 39.0\left(\mathrm{CH}_{2}\right), 33.1(\mathrm{C}), 30.6\left(\mathrm{CH}_{2}\right)$, $25.7\left(2 \mathrm{CH}_{3}\right) ; \delta$ (major 2$)=215.7(\mathrm{C}), 171.1(\mathrm{C}), 171.1(\mathrm{C}), 139.2(\mathrm{C})$ $131.9(2 \mathrm{CH}), 127.7(\mathrm{CH}), 120.6(2 \mathrm{CH}), 81.2(\mathrm{C}), 62.8(\mathrm{C}), 53.8\left(\mathrm{CH}_{3}\right)$ $53.1\left(\mathrm{CH}_{2}\right), 43.7\left(\mathrm{CH}_{2}\right), 43.2(\mathrm{CH}), 33.9\left(2 \mathrm{CH}_{3}\right), 30.6(\mathrm{C}), 29.7\left(\mathrm{CH}_{2}\right) ; \delta$ (minor $1+$ minor 2$)=216.5(\mathrm{C}), 216.4$ (C), 174.6 (C), 172.4 (C), 171.4 (C), $171.3(\mathrm{C}), 138.7(\mathrm{C}), 138.6(\mathrm{C}), 134.1(2 \mathrm{CH}), 129.6(2 \mathrm{CH}), 129.4(2$ (CH), $128.7(2 \mathrm{CH}), 128.6(\mathrm{CH}), 128.1(\mathrm{CH}), 128.01(\mathrm{CH}), 82.6(\mathrm{C}), 82.3$ (C), $62.9(\mathrm{C}), 62.3(\mathrm{C}), 55.2\left(\mathrm{CH}_{3}\right), 54.8\left(\mathrm{CH}_{3}\right), 53.6\left(\mathrm{CH}_{2}\right), 53.5\left(\mathrm{CH}_{2}\right)$ $45.6\left(\mathrm{CH}_{2}\right), 45,29(\mathrm{CH}), 40.6\left(\mathrm{CH}_{2}\right), 32.6(\mathrm{C}), 34.6\left(2 \mathrm{CH}_{3}\right), 33.3\left(2 \mathrm{CH}_{3}\right)$, $32.5(\mathrm{C}), 30.0\left(\mathrm{CH}_{2}\right), 29.9\left(\mathrm{CH}_{2}\right)$.

HRMS (ESI+): $m / z[\mathrm{M}+\mathrm{H}]^{+}$calcd for $\mathrm{C}_{19} \mathrm{H}_{24} \mathrm{NO}_{5}$ : 346.1649; found: 346.1646 .

\section{Methyl 2-(Furan-2-ylmethyl)-3-hydroxy-1,7-dioxo-5-phenyl-2- azaspiro[5.6]dodecane-3-carboxylate (3hd)}

Following the general procedure, the reaction of $N$-(furan-2-ylmethyl)-2-oxo-cycloheptanecarboxamide $(163 \mathrm{mg}, 0.69 \mathrm{mmol})$ with methyl (E)-2-oxo-4-phenylbut-3-enoate (2d; $144 \mathrm{mg}, 0.76 \mathrm{mmol}$ ) in the presence of PS-BEMP (32 mg, $10 \mathrm{~mol}$ ) in THF ( $1.5 \mathrm{~mL}$ ) at r.t. for 9 $\mathrm{h}$ afforded the product $3 \mathrm{hd}$ as a white solid $(262 \mathrm{mg}, 88 \%$ ). The crude product was obtained with 3:1.50:1.5:1 dr. One diastereomer was separated.

First fraction: one diastereomer was isolated as a yellow oil $(110 \mathrm{mg}$ $42 \%) ; R_{f}=0.52\left(\mathrm{Et}_{2} \mathrm{O} / \mathrm{PE}, 7: 3\right)$.

${ }^{1} \mathrm{H} \mathrm{NMR}\left(300 \mathrm{MHz}, \mathrm{CDCl}_{3}\right): \delta=7.32-7.31(\mathrm{~m}, 1 \mathrm{H}), 7.30-7.29(\mathrm{~m}, 1 \mathrm{H})$, $7.28(\mathrm{~d}, J=1.7 \mathrm{~Hz}, 2 \mathrm{H}), 7.16-7.11(\mathrm{~m}, 2 \mathrm{H}), 6.30(\mathrm{~d}, J=1.4 \mathrm{~Hz}, 2 \mathrm{H})$ $4.60(\mathrm{~d}, J=15.9 \mathrm{~Hz}, 1 \mathrm{H}), 4.39(\mathrm{~s}, 1 \mathrm{H}), 4.29(\mathrm{~d}, J=15.9 \mathrm{~Hz}, 1 \mathrm{H}), 3.75(\mathrm{~s}$ $3 \mathrm{H}), 3.41(\mathrm{dd}, J=13.7,2.1 \mathrm{~Hz}, 1 \mathrm{H}), 3.14(\mathrm{t}, J=13.4 \mathrm{~Hz}, 1 \mathrm{H}), 2.42(\mathrm{dt}$ $J=9.5,6.4 \mathrm{~Hz}, 1 \mathrm{H}), 2.09-2.03(\mathrm{~m}, 3 \mathrm{H}), 1.84(\mathrm{dd}, J=13.2,2.2 \mathrm{~Hz}, 1 \mathrm{H})$ $1.77-1.64(\mathrm{~m}, 2 \mathrm{H}), 1.35-1.25(\mathrm{~m}, 1 \mathrm{H}), 1.17(\mathrm{~d}, J=15.0 \mathrm{~Hz}, 1 \mathrm{H}), 0.92-$ $0.82(\mathrm{~m}, 2 \mathrm{H})$.

${ }^{13} \mathrm{C} \operatorname{NMR}\left(75 \mathrm{MHz}, \mathrm{CDCl}_{3}\right): \delta=213.1$ (C), 172.7 (C), $170.2(\mathrm{C}), 150.9$ (C), $141.7(\mathrm{CH}), 138.0(\mathrm{C}), 129.3(2 \mathrm{CH}), 128.5(2 \mathrm{CH}), 127.9(\mathrm{CH}), 110.5$ (CH), $108.4(\mathrm{CH}), 85.0(\mathrm{COH}), 64.4(\mathrm{C}), 54.2\left(\mathrm{CH}_{3}\right), 45.5\left(\mathrm{CH}_{2}\right), 44.2$ (CH), $40.7\left(\mathrm{CH}_{2}\right), 36.0\left(\mathrm{CH}_{2}\right), 35.0\left(\mathrm{CH}_{2}\right), 30.9\left(\mathrm{CH}_{2}\right), 25.6\left(\mathrm{CH}_{2}\right), 25.4$ $\left(\mathrm{CH}_{2}\right)$.

Second fraction: a mixture of two diastereomers was obtained (105 $\mathrm{mg}, 40 \%) ; R_{f}=0.57\left(\mathrm{Et}_{2} \mathrm{O} / \mathrm{PE}, 7: 3\right)$.

${ }^{1} \mathrm{H} \mathrm{NMR}\left(300 \mathrm{MHz}, \mathrm{CDCl}_{3}\right): \delta=7.33-7.25(\mathrm{~m}, 8 \mathrm{H}), 7.13-7.08(\mathrm{~m}, 4 \mathrm{H})$ $6.32-6.27(\mathrm{~m}, 3 \mathrm{H}), 6.24(\mathrm{~d}, J=3.2 \mathrm{~Hz}, 1 \mathrm{H}), 4.75(\mathrm{dd}, J=20.1,3.0 \mathrm{~Hz}, 2$ $\mathrm{H}), 4.57(\mathrm{~d}, J=8.0 \mathrm{~Hz}, 3 \mathrm{H}), 4.28-4.12(\mathrm{~m}, 3 \mathrm{H}), 3.71(\mathrm{~s}, 3 \mathrm{H}), 3.70(\mathrm{~s}, 3$ H), $2.96(\mathrm{t}, J=13.9 \mathrm{~Hz}, 1 \mathrm{H}), 2.78(\mathrm{t}, J=14.5 \mathrm{~Hz}, 1 \mathrm{H}), 2.51(\mathrm{dd}, J=18.8$ $10.3 \mathrm{~Hz}, 2 \mathrm{H}), 2.26-2.12(\mathrm{~m}, 3 \mathrm{H}), 2.02(\mathrm{dd}, J=13.7,2.8 \mathrm{~Hz}, 1 \mathrm{H}), 1.84-$ $1.66(\mathrm{~m}, 11 \mathrm{H}), 1.60-1.45(\mathrm{~m}, 5 \mathrm{H})$.

${ }^{13} \mathrm{C} \operatorname{NMR}\left(75 \mathrm{MHz}, \mathrm{CDCl}_{3}\right): \delta=213.3(\mathrm{C}), 213.2(\mathrm{C}), 173.0(\mathrm{C}), 172.4(\mathrm{C})$, 171.7 (C), 171.5 (C), 150.9 (C), 150.8 (C), $141.6(2 \mathrm{CH}), 137.8(2 \mathrm{C})$ $128.9(2 \mathrm{CH}), 128.8(2 \mathrm{CH}), 128.4(2 \mathrm{CH}), 127.6(2 \mathrm{CH}), 127.5(\mathrm{CH})$ $121.0(\mathrm{CH}), 110.6(\mathrm{CH}), 110.5(\mathrm{CH}), 108.9(\mathrm{CH}), 108.5(\mathrm{CH}), 85.0$ $(\mathrm{COH}), 84.4(\mathrm{COH}), 63.8(\mathrm{C}), 63.3(\mathrm{C}), 54.3\left(\mathrm{CH}_{3}\right), 54.2\left(\mathrm{CH}_{3}\right), 45.8$ $\left(\mathrm{CH}_{2}\right), 45.7\left(\mathrm{CH}_{2}\right), 42.4(\mathrm{CH}), 42.0(\mathrm{CH}), 39.5\left(\mathrm{CH}_{2}\right), 37.7\left(\mathrm{CH}_{2}\right), 35.6$ $\left(\mathrm{CH}_{2}\right), 35.0\left(\mathrm{CH}_{2}\right), 30.98\left(\mathrm{CH}_{2}\right), 30.92\left(\mathrm{CH}_{2}\right), 28.2\left(\mathrm{CH}_{2}\right), 27.7\left(\mathrm{CH}_{2}\right), 23.9$ $\left(\mathrm{CH}_{2}\right), 24.0\left(\mathrm{CH}_{2}\right), 23.9\left(\mathrm{CH}_{2}\right), 23.8\left(\mathrm{CH}_{2}\right)$.

HRMS (ESI+): $m / z[\mathrm{M}+\mathrm{H}]^{+}$calcd for $\mathrm{C}_{24} \mathrm{H}_{27} \mathrm{NO}_{6}: 426.1911$; found: 426.1907.
1'-Allyl-6'-hydroxyspiro[indene-2,3'-piperidine]-1,2'(3H)-dione (3ia)

Following the general procedure, the reaction of 1-oxoindan-2-carboxylic acid allylamide ( $100 \mathrm{mg}, 0.46 \mathrm{mmol}$ ) with acrolein (2a; 33.80 $\mu \mathrm{L}, 0.55 \mathrm{mmol}$ ) in the presence of PS-BEMP (40 mg, $10 \mathrm{~mol} \%$ ) in THF $(1 \mathrm{~mL})$ at r.t. for $24 \mathrm{~h}$ afforded the product 3ia as a white solid (124 $\mathrm{mg}, 89 \%$ ). The crude product was obtained with $1: 1 \mathrm{dr} ; R_{f}=0.24$ $\left(\mathrm{Et}_{2} \mathrm{O}, \mathrm{PE}, 8: 2\right)$. Data given are for the mixture of two diastereomers.

${ }^{1} \mathrm{H} \mathrm{NMR}\left(300 \mathrm{MHz}, \mathrm{CDCl}_{3}\right): \delta=7.77(\mathrm{~d}, J=7.7 \mathrm{~Hz}, 2 \mathrm{H}), 7.63(\mathrm{t}, J=7.4$ $\mathrm{Hz}, 2 \mathrm{H}), 7.48(\mathrm{~d}, J=7.7 \mathrm{~Hz}, 2 \mathrm{H}), 7.40(\mathrm{t}, J=7.7 \mathrm{~Hz}, 2 \mathrm{H}), 5.92-5.78(\mathrm{~m}$, $2 \mathrm{H}), 5.28-5.15(\mathrm{~m}, 5 \mathrm{H}), 5.04(\mathrm{~s}, 2 \mathrm{H}), 4.38-4.35(\mathrm{~m}, 1 \mathrm{H}), 4.34-4.30$ (m, $1 \mathrm{H}), 3.98-3.84(\mathrm{~m}, 4 \mathrm{H}), 3.75-3.65(\mathrm{~m}, 2 \mathrm{H}), 2.97(\mathrm{~d}, J=16.9 \mathrm{~Hz}, 2$ H), 2.49 (ddd, $J=14.2,10.5,5.1 \mathrm{~Hz}, 2 \mathrm{H}$ ), 2.17 (s, $3 \mathrm{H}), 1.87$ (dt, $J=14.0$, $5.9 \mathrm{~Hz}, 2 \mathrm{H})$.

$\left.{ }^{13} \mathrm{C} \mathrm{NMR} \mathrm{(75} \mathrm{MHz,} \mathrm{CDCl}\right)_{3}$ ): $\delta$ (diastereomer 1) = 204.96 (C), 169.97 (C), $153.72(\mathrm{C}), 135.10(\mathrm{CH}), 134.11(\mathrm{C}), 132.75(\mathrm{CH}), 127.56(\mathrm{CH}), 126.30$ $(\mathrm{CH}), 124.73(\mathrm{CH}), 116.46\left(\mathrm{CH}_{2}\right), 79.19(\mathrm{CHOH}), 60.42\left(\mathrm{CH}_{2}\right), 56.20(\mathrm{C})$, $40.60\left(\mathrm{CH}_{2}\right), 26.52\left(\mathrm{CH}_{2}\right), 26.44\left(\mathrm{CH}_{2}\right) ; \delta$ (diastereomer 2$)=206.42(\mathrm{C})$, $169.71(\mathrm{C}), 153.84(\mathrm{C}), 135.68(\mathrm{CH}), 135.20(\mathrm{C}), 132.73(\mathrm{CH}), 127.84$ $(\mathrm{CH}), 126.50(\mathrm{CH}), 124.80(\mathrm{CH}), 117.25\left(\mathrm{CH}_{2}\right), 78.81(\mathrm{CHOH}), 57.09(\mathrm{C})$, $46.95\left(\mathrm{CH}_{2}\right), 41.17\left(\mathrm{CH}_{2}\right), 26.73\left(\mathrm{CH}_{2}\right), 28.12\left(\mathrm{CH}_{2}\right)$.

HRMS (ESI+): $\mathrm{m} / z[\mathrm{M}+\mathrm{H}]^{+}$calcd for $\mathrm{C}_{16} \mathrm{H}_{17} \mathrm{NO}_{3} \mathrm{Na}$ : 294.1101; found: 294.1104.

6'-Hydroxy-1'-phenyl-3,4-dihydro-1H-spiro[naphthalene-2,3'piperidine]-1,2'-dione (3ja)

Following the general procedure, the reaction of $\mathrm{N}$-allyl-1-oxo-1-phenyl-1,2,3,4-tetrahydronaphthalene-2-carboxamide $(33 \mathrm{mg}, \quad 0.12$ mmol) with acrolein (2a; $9 \mu \mathrm{L}, 0.13 \mathrm{mmol}$ ) in the presence of PSBEMP ( $5 \mathrm{mg}, 10 \mathrm{~mol} \%)$ in THF $(0.5 \mathrm{~mL})$ at r.t. for $5 \mathrm{~h}$ afforded the product 3ja as a white solid (39 mg, 99\%). The crude product was obtained with 2:1 dr; $R_{f}=0.15\left(\mathrm{Et}_{2} \mathrm{O} / \mathrm{PE}, 7: 3\right)$. Data given are for the mixture of two diastereomers.

${ }^{1} \mathrm{H} \mathrm{NMR}\left(400 \mathrm{MHz}, \mathrm{CDCl}_{3}\right): \delta=8.07-8.03(\mathrm{~m}, 2 \mathrm{H}), 7.51(\mathrm{dd}, J=7.4,1.3$ $\mathrm{Hz}, 1 \mathrm{H}), 7.48$ (dd, $J=7.5,1.3 \mathrm{~Hz}, 1 \mathrm{H}), 7.43-7.41(\mathrm{~m}, 1 \mathrm{H}), 7.40$ (dd, $J=$ 3.0, $1.1 \mathrm{~Hz}, 2 \mathrm{H}), 7.38(\mathrm{~d}, J=3.1 \mathrm{~Hz}, 2 \mathrm{H}), 7.34-7.30(\mathrm{~m}, 6 \mathrm{H}), 7.29(\mathrm{~d}$ $J=1.1 \mathrm{~Hz}, 2 \mathrm{H}), 7.24(\mathrm{~s}, 1 \mathrm{H}), 5.29(\mathrm{~s}, 1 \mathrm{H}), 5.24(\mathrm{~s}, 1 \mathrm{H}), 3.74(\mathrm{~d}, J=7.9$ $\mathrm{Hz}, 1 \mathrm{H}), 3.15(\mathrm{~d}, J=4.5 \mathrm{~Hz}, 1 \mathrm{H}), 3.12(\mathrm{dd}, J=7.6,4.1 \mathrm{~Hz}, 1 \mathrm{H}), 3.11-$ $3.01(\mathrm{~m}, 3 \mathrm{H}), 2.99-2.89(\mathrm{~m}, 1 \mathrm{H}), 2.62-2.55(\mathrm{~m}, 1 \mathrm{H}), 2.40(\mathrm{tt} J=5.4$ $3.6 \mathrm{~Hz}, 1 \mathrm{H}), 2.32(\mathrm{~m}, 1 \mathrm{H}), 2.32-2.25(\mathrm{~m}, 1 \mathrm{H}), 2.18-2.15(\mathrm{~m}, 1 \mathrm{H})$, $2.15-2.10(\mathrm{~m}, 2 \mathrm{H}), 2.10-2.00(\mathrm{~m}, 2 \mathrm{H}), 1.96(\mathrm{dd}, J=7.7,5.1 \mathrm{~Hz}, 1 \mathrm{H})$, 1.90 (ddd, $J=7.0,6.0,3.0 \mathrm{~Hz}, 1 \mathrm{H}$ ).

${ }^{13} \mathrm{C}$ NMR $\left(75 \mathrm{MHz}, \mathrm{CDCl}_{3}\right): \delta=198.5(\mathrm{C}), 196.9(\mathrm{C}), 170.89(\mathrm{C}), 170.87$ (C), 143.6 (C), 143.1 (C), 141.7 (C), 140.6 (C), 134.1 (CH), 133.7 (CH), $131.2(\mathrm{C}), 130.4(\mathrm{C}), 129.28(2 \mathrm{CH}), 129.22(2 \mathrm{CH}), 128.8(\mathrm{CH}), 128.7$ $(\mathrm{CH}), 128.4(\mathrm{CH}), 128.2(2 \mathrm{CH}), 128.0(2 \mathrm{CH}), 127.9(\mathrm{CH}), 127.5(2 \mathrm{CH})$, 126.97 (CH), $126.91(\mathrm{CH}), 82.2(\mathrm{CHOH}), 81.8(\mathrm{CHOH}), 54.3(\mathrm{C}), 54.1$ (C), $32.6\left(\mathrm{CH}_{2}\right), 31.7\left(\mathrm{CH}_{2}\right), 27.5\left(\mathrm{CH}_{2}\right), 26.1\left(\mathrm{CH}_{2}\right), 25.6\left(\mathrm{CH}_{2}\right), 25.0$ $\left(\mathrm{CH}_{2}\right), 24.6\left(\mathrm{CH}_{2}\right), 23.4\left(\mathrm{CH}_{2}\right)$.

HRMS (ESI+): $m / z[\mathrm{M}+\mathrm{H}]^{+}$calcd for $\mathrm{C}_{20} \mathrm{H}_{20} \mathrm{NO}_{3}: 322.1438$; found: 322.1437 .

1'-Allyl-6'-hydroxy-3,4-dihydro-1H-spiro[naphthalene-2,3'-piperidine]-1,2'-dione (3ka)

Following the general procedure, the reaction of $\mathrm{N}$-allyl-1-oxo1,2,3,4-tetrahydronaphthalene-2-carboxamide ( $160 \mathrm{mg}, 0.69 \mathrm{mmol})$ with acrolein (2a; $50.70 \mu \mathrm{L}, 0.759 \mathrm{mmol}$ ) in the presence of PS-BEMP (31 mg, $10 \mathrm{~mol} \%)$ in THF $(1.5 \mathrm{~mL})$ at r.t. for $24 \mathrm{~h}$ afforded the product 3ka as a yellow solid $(219 \mathrm{mg}, 86 \%)$. The crude product was obtained with 2:1 dr. 
First fraction: one diastereomer was isolated as a yellow solid ( $53 \mathrm{mg}$, $28 \%) ; R_{f}=0.29(\mathrm{EtOAc} / \mathrm{PE}, 1: 1)$.

${ }^{1} \mathrm{H} \mathrm{NMR}\left(400 \mathrm{MHz}, \mathrm{CDCl}_{3}\right): \delta=7.95(\mathrm{dd}, J=7.8,2.8 \mathrm{~Hz}, 1 \mathrm{H}), 7.42(\mathrm{dtd}$, $J=11.2,7.5,2.2 \mathrm{~Hz}, 1 \mathrm{H}), 7.24(\mathrm{dd}, J=15.8,7.9 \mathrm{~Hz}, 1 \mathrm{H}), 7.18(\mathrm{dd}, J=$ $16.8,6.4 \mathrm{~Hz}, 1 \mathrm{H}), 5.87-5.66(\mathrm{~m}, 1 \mathrm{H}), 5.33-5.08(\mathrm{~m}, 2 \mathrm{H}), 4.98(\mathrm{~s}, 1 \mathrm{H})$, $4.86(\mathrm{t}, J=4.7 \mathrm{~Hz}, 1 \mathrm{H}), 4.32$ (ddd, $J=15.6,10.7,4.8 \mathrm{~Hz}, 1 \mathrm{H}), 3.83$ (dd, $J=15.7,6.3 \mathrm{~Hz}, 1 \mathrm{H}), 3.03-2.75(\mathrm{~m}, 3 \mathrm{H}), 2.39-2.13(\mathrm{~m}, 1 \mathrm{H}), 2.08-1.70$ $(\mathrm{m}, 4 \mathrm{H})$.

${ }^{13} \mathrm{C}$ NMR $\left(75 \mathrm{MHz}, \mathrm{CDCl}_{3}\right): \delta=198.59(\mathrm{C}), 170.33(\mathrm{C}), 143.61(\mathrm{C})$ $134.16(\mathrm{CH}), 133.63(\mathrm{CH}), 131.31(\mathrm{C}), 128.82(\mathrm{CH}), 128.49(\mathrm{CH})$, $127.04(\mathrm{CH}), 117.27\left(\mathrm{CH}_{2}\right), 79.46(\mathrm{CHOH}), 54.22(\mathrm{C}), 46.96\left(\mathrm{CH}_{2}\right)$, $31.75\left(\mathrm{CH}_{2}\right), 27.71\left(\mathrm{CH}_{2}\right), 25.10\left(\mathrm{CH}_{2}\right), 24.84\left(\mathrm{CH}_{2}\right)$.

Second fraction: one diastereomer was isolated as a yellow solid (109 $\mathrm{mg}, 58 \%) ; \mathrm{mp} 51^{\circ} \mathrm{C} ; R_{f}=0.2$ (EtOAc/PE, 1:1).

${ }^{1} \mathrm{H} \mathrm{NMR}\left(300 \mathrm{MHz}, \mathrm{CDCl}_{3}\right): \delta=8.03(\mathrm{~d}, J=7.8 \mathrm{~Hz}, 1 \mathrm{H}), 7.49(\mathrm{td}, J=7.4$ $1.0 \mathrm{~Hz}, 1 \mathrm{H}), 7.31(\mathrm{t}, J=7.3 \mathrm{~Hz}, 1 \mathrm{H}), 7.22(\mathrm{~d}, J=4.6 \mathrm{~Hz}, 1 \mathrm{H}), 5.92-5.78$ $(\mathrm{m}, 1 \mathrm{H}), 5.27-5.14(\mathrm{~m}, 2 \mathrm{H}), 4.98-4.90(\mathrm{~m}, 1 \mathrm{H}), 4.39(\mathrm{dd}, J=15.3,4.9$ $\mathrm{Hz}, 1 \mathrm{H}), 3.93(\mathrm{dd}, J=15.3,6.8 \mathrm{~Hz}, 1 \mathrm{H}), 3.44(\mathrm{~d}, J=9.8 \mathrm{~Hz}, 1 \mathrm{H}), 3.04$ (dt, $J=9.9,4.9 \mathrm{~Hz}, 1 \mathrm{H}), 2.97-2.83(\mathrm{~m}, 1 \mathrm{H}), 2.48-2.37(\mathrm{~m}, 1 \mathrm{H}), 2.16$ (ddd, $J=13.6,8.7,4.5 \mathrm{~Hz}, 1 \mathrm{H}), 2.07-1.97(\mathrm{~m}, 1 \mathrm{H}), 1.96-1.74(\mathrm{~m}, 3 \mathrm{H})$. ${ }^{13} \mathrm{C}$ NMR $\left(75 \mathrm{MHz}, \mathrm{CDCl}_{3}\right): \delta=199.01(\mathrm{C}), 170.76(\mathrm{C}), 144.04(\mathrm{C})$, $134.59(\mathrm{CH}), 134.05(\mathrm{CH}), 131.73(\mathrm{C}), 129.25(\mathrm{CH}), 128.91(\mathrm{CH})$ $127.47(\mathrm{CH}), 117.69\left(\mathrm{CH}_{2}\right), 79.89(\mathrm{CHOH}), 54.65(\mathrm{C}), 47.39\left(\mathrm{CH}_{2}\right)$, $32.18\left(\mathrm{CH}_{2}\right), 28.14\left(\mathrm{CH}_{2}\right), 25.53\left(\mathrm{CH}_{2}\right), 25.27\left(\mathrm{CH}_{2}\right)$.

HRMS (ESI+): $m / z[\mathrm{M}+\mathrm{H}]^{+}$calcd for $\mathrm{C}_{17} \mathrm{H}_{20} \mathrm{NO}_{3}: 286.1438$; found: 286.1437.

\section{8a-Hydroxy-10-phenylhexahydro-2H,5H-2,4a-(epiminometh- ano)chromen-9-one (5la)}

Following the general procedure, the reaction of of 2-oxo- $\mathrm{N}$-phenylcyclohexanecarboxamide $(\mathbf{1 1} ; 100 \mathrm{mg}, 0.46 \mathrm{mmol})$ with acrolein $(\mathbf{2 a}$; $34 \mu \mathrm{L}, 0.50 \mathrm{mmol}$ ) in the presence of PS-BEMP (21 mg, $10 \mathrm{~mol} \%$ ) in THF $(1 \mathrm{~mL})$ heated at $55-60{ }^{\circ} \mathrm{C}$ for $24 \mathrm{~h}$, afforded the product $5 \mathbf{l a}$ as a yellow oil ( $88 \mathrm{mg}, 70 \%$ ). The crude product was obtained with $2: 1 \mathrm{dr}$; $R_{f}=0.34\left(\mathrm{Et}_{2} \mathrm{O} / \mathrm{PE}, 7: 3\right)$. Data given are for the mixture of two diastereomers.

${ }^{1} \mathrm{H} \mathrm{NMR}\left(300 \mathrm{MHz}, \mathrm{CDCl}_{3}\right): \delta=7.33-7.30(\mathrm{~m}, 4 \mathrm{H}), 7.28(\mathrm{~d}, J=1.6 \mathrm{~Hz}, 1$ $\mathrm{H}), 7.25(\mathrm{~d}, J=3.0 \mathrm{~Hz}, 1 \mathrm{H}), 7.17$ (ddd, $J=6.9,5.6,1.4 \mathrm{~Hz}, 2 \mathrm{H}), 5.44(\mathrm{t}$, $J=2.2 \mathrm{~Hz}, 1 \mathrm{H}), 5.36(\mathrm{t}, J=2.2 \mathrm{~Hz}, 1 \mathrm{H}), 3.71$ (ddd, $J=6.6,4.2,2.6 \mathrm{~Hz}, 2$ H), 2.38-2.26 (m, $1 \mathrm{H}), 2.09-2.22(\mathrm{~m}, 4 \mathrm{H}), 2.01-1.89(\mathrm{~m}, 3 \mathrm{H}), 1.76-$ $1.83(\mathrm{~m}, 5 \mathrm{H}), 1.61-1.32(\mathrm{~m}, 13 \mathrm{H})$.

${ }^{13} \mathrm{C}$ NMR $\left(75 \mathrm{MHz}, \mathrm{CDCl}_{3}\right.$ ): $\delta$ (major) $=172.49$ (C), 139.40 (C), 128.95 $(2 \mathrm{CH}), 126.16(2 \mathrm{CH}), 123.95(\mathrm{CH}), 98.22(\mathrm{COH}), 84.24(\mathrm{CH}), 48.07(\mathrm{C})$, $38.57\left(\mathrm{CH}_{2}\right), 28.30\left(\mathrm{CH}_{2}\right), 27.93\left(\mathrm{CH}_{2}\right), 22.67\left(\mathrm{CH}_{2}\right), 21.85\left(\mathrm{CH}_{2}\right), 20.10$ $\left(\mathrm{CH}_{2}\right) ; \delta($ minor $)=172.12(\mathrm{C}), 138.99(\mathrm{C}), 128.93(2 \mathrm{CH}), 126.12(2$ $\mathrm{CH}), 124.23(\mathrm{CH}), 99.54(\mathrm{COH}), 84.30(\mathrm{CH}), 47.00(\mathrm{C}), 33.33\left(\mathrm{CH}_{2}\right)$, $28.07\left(\mathrm{CH}_{2}\right), 26.03\left(\mathrm{CH}_{2}\right), 22.95\left(\mathrm{CH}_{2}\right), 21.99\left(\mathrm{CH}_{2}\right), 20.29\left(\mathrm{CH}_{2}\right)$.

\section{Methyl 8a-Hydroxy-9-oxo-4,10-diphenylhexahydro-2H,5H-2,4a-} (epiminomethano)chromene-2-carboxylate (5ld)

Following the general procedure, the reaction of 2-oxo- $\mathrm{N}$-phenylcyclohexanecarboxamide (11; $150 \mathrm{mg}, 0.69 \mathrm{mmol}$ ) with methyl $(E)$-2oxo-4-phenylbut-3-enoate (2d; $145 \mathrm{mg}, 0.76 \mathrm{mmol})$ in the presence of PS-BEMP (32 mg, $10 \mathrm{~mol} \%)$ in THF (1.5 mL) at r.t. for $30 \mathrm{~h}$ afforded the product 5ld as a white solid (160.5 mg, 58\%). The crude product was obtained with $3: 1 \mathrm{dr} ; R_{f}=0.52\left(\mathrm{Et}_{2} \mathrm{O} / \mathrm{PE}, 7: 3\right)$. The major diastereomer partially crystallized as white needles for X-ray analysis; $\mathrm{mp}$ $163{ }^{\circ} \mathrm{C}$. Data given are for the mixture of two diastereomers.
${ }^{1} \mathrm{H} \mathrm{NMR}\left(300 \mathrm{MHz}, \mathrm{CDCl}_{3}\right): \delta=7.46-7.27(\mathrm{~m}, 20 \mathrm{H}), 5.26(\mathrm{~s}, 1 \mathrm{H}), 4.19$ (s, $1 \mathrm{H}), 3.87$ (dd, $J=10.4,3.6 \mathrm{~Hz}, 1 \mathrm{H}), 3.63(\mathrm{dd}, J=10.3,5.3 \mathrm{~Hz}, 1 \mathrm{H})$, $3.45(\mathrm{~d}, J=2.5 \mathrm{~Hz}, 1 \mathrm{H}), 3.39(\mathrm{~s}, 3 \mathrm{H}), 3.38(\mathrm{~s}, 1 \mathrm{H}), 3.32(\mathrm{~d}, J=2.5 \mathrm{~Hz}, 1$ H), 2.99 (dd, $J=13.8,10.5 \mathrm{~Hz}, 1 \mathrm{H}$ ), 2.80 (dd, $J=13.9,3.8 \mathrm{~Hz}, 2 \mathrm{H}$ ), 2.68 (dd, $J=13.5,5.5 \mathrm{~Hz}, 1 \mathrm{H}), 2.19-2.06(\mathrm{~m}, 3 \mathrm{H}), 1.68-1.37(\mathrm{~m}, 13 \mathrm{H})$.

${ }^{13} \mathrm{C}$ NMR $\left(75 \mathrm{MHz}, \mathrm{CDCl}_{3}\right): \delta$ (minor) = 171.6 (C), 166.3 (C), 140.9 (C), $137.94(\mathrm{C}), 129.0(2 \mathrm{CH}), 128.9(2 \mathrm{CH}), 128.7(\mathrm{CH}), 128.6(\mathrm{CH}), 128.1$ $(2 \mathrm{CH}), 127.4(2 \mathrm{CH}), 100.4(\mathrm{C}), 89.11(\mathrm{C}), 52.7(\mathrm{CH}), 51.8\left(\mathrm{CH}_{3}\right), 42.3$ $\left(\mathrm{CH}_{2}\right), 36.8(\mathrm{CH}), 33.4\left(\mathrm{CH}_{2}\right), 23.4\left(\mathrm{CH}_{2}\right), 21.9\left(\mathrm{CH}_{2}\right), 19.5\left(\mathrm{CH}_{2}\right) ; \delta(\mathrm{ma}-$ jor $)=171.5(\mathrm{C}), 166.7(\mathrm{C}), 140.7(\mathrm{C}), 137.96(\mathrm{C}), 129.1(2 \mathrm{CH}), 128.8(2$ CH), 128.5 (2 CH), 128.3 (C), 127.9 (C), $127.2(2 \mathrm{CH}), 99.2(\mathrm{C}), 89.11$ (C), $52.9(\mathrm{CH}), 52.8\left(\mathrm{CH}_{3}\right), 40.6\left(\mathrm{CH}_{2}\right), 38.7(\mathrm{CH}), 38.4\left(\mathrm{CH}_{2}\right), 27.1\left(\mathrm{CH}_{2}\right)$, $22.3\left(\mathrm{CH}_{2}\right), 21.4\left(\mathrm{CH}_{2}\right)$.

Methyl 10-(Furan-2-ylmethyl)-8a-hydroxy-9-oxo-4-phenylhexahydro-2H,5H-2,4a-(epiminomethano)chromene-2-carboxylate (5md)

Following the general procedure, the reaction of $N$-(furan-2ylmethyl)-2-oxocyclohexanecarboxamide (1m; $210 \mathrm{mg}, 0.95 \mathrm{mmol})$ with methyl (E)-2-oxo-4-phenylbut-3-enoate (2d; $198.75 \mathrm{mg}, 1.04 \mathrm{mmol}$ ) in the presence of PS-BEMP $(41 \mathrm{mg}, 10 \mathrm{~mol} \%)$ in THF $(2 \mathrm{~mL})$ at r.t. for 5 $\mathrm{h}$ afforded the product $5 \mathrm{md}$ as a yellow solid $(250 \mathrm{mg}, 64 \%)$. The crude product was obtained with 6:1:1 dr; $R_{f}=0.47\left(\mathrm{Et}_{2} \mathrm{O} / \mathrm{PE}, 7: 3\right)$. Data given are for the mixture of three diastereomers.

\section{Three diastereomers:}

${ }^{1} \mathrm{H} \mathrm{NMR}\left(300 \mathrm{MHz}, \mathrm{CDCl}_{3}\right): \delta$ (characteristic peaks, major) $=7.37$ (d, $J=1.7 \mathrm{~Hz}, 1 \mathrm{H}), 7.28(\mathrm{br} \mathrm{s}, 1 \mathrm{H}), 7.24(\mathrm{br} \mathrm{s}, 2 \mathrm{H}), 7.05(\mathrm{~d}, J=2.0 \mathrm{~Hz}, 1 \mathrm{H})$, $7.02(\mathrm{~d}, J=1.4 \mathrm{~Hz}, 1 \mathrm{H}), 5.31(\mathrm{~s}, 1 \mathrm{H}), 6.33(\mathrm{dd}, J=3.2,1.9 \mathrm{~Hz}, 1 \mathrm{H}), 6.27$ $(\mathrm{d}, J=3.2 \mathrm{~Hz}, 1 \mathrm{H}), 5.11(\mathrm{~d}, J=15.9 \mathrm{~Hz}, 1 \mathrm{H}), 4.72(\mathrm{~d}, J=15.9 \mathrm{~Hz}, 1 \mathrm{H})$, $3.84(\mathrm{~s}, 3 \mathrm{H}), 3.68(\mathrm{dd}, J=10.4,3.6 \mathrm{~Hz}, 1 \mathrm{H}), 2.89(\mathrm{dd}, J=13.0,4.6 \mathrm{~Hz}, 1$ H), 2.51 (dd, $J=13.8,3.6 \mathrm{~Hz}, 1 \mathrm{H})$.

${ }^{13} \mathrm{C}$ NMR $\left(75 \mathrm{MHz}, \mathrm{CDCl}_{3}\right): \delta$ (major) = $171.6(\mathrm{C}), 167.1(\mathrm{C}), 150.0(\mathrm{C})$, 142.2 (CH), $140.4(\mathrm{C}), 128.7(2 \mathrm{CH}), 128.1(2 \mathrm{CH}), 127.0(\mathrm{CH}), 110.3$ $(\mathrm{CH}), 108.8(\mathrm{CH}), 98.5(\mathrm{C}), 86.4(\mathrm{C}), 53.1\left(\mathrm{CH}_{3}\right), 52.2(\mathrm{C}), 40.8\left(\mathrm{CH}_{2}\right)$, 38.8 (CH), $38.1\left(\mathrm{CH}_{2}\right), 37.9\left(\mathrm{CH}_{2}\right), 27.0\left(\mathrm{CH}_{2}\right), 22.3\left(\mathrm{CH}_{2}\right), 21.4\left(\mathrm{CH}_{2}\right) ; \delta$ (minor 1 + minor 2) $=172.9(\mathrm{C}), 171.8(\mathrm{C}), 166.7(\mathrm{C}), 163.3(\mathrm{C}), 151.2$ (C), $150.1(\mathrm{C}), 142.3(\mathrm{CH}), 142.0(\mathrm{CH}), 140.7(\mathrm{C}), 139.9(\mathrm{C}), 129.5(2$ $\mathrm{CH}), 128.7(2 \mathrm{CH}), 128.3(2 \mathrm{CH}), 127.8(2 \mathrm{CH}), 127.2(\mathrm{CH}), 127.1(\mathrm{CH})$ 114.0 (CH), $110.4(\mathrm{CH}), 110.4(\mathrm{CH}), 107.8(\mathrm{CH}), 99.8(\mathrm{C}), 99.1(\mathrm{C}), 86.5$ (C), $82.5(\mathrm{C}), 54.4\left(\mathrm{CH}_{3}\right), 52.6\left(\mathrm{CH}_{3}\right), 51.6(\mathrm{C}), 50.2(\mathrm{C}), 44.7(\mathrm{CH}), 42.2$ $\left(\mathrm{CH}_{2}\right), 41.8\left(\mathrm{CH}_{2}\right), 41.3\left(\mathrm{CH}_{2}\right), 38.4\left(\mathrm{CH}_{2}\right), 36.8(\mathrm{CH}), 36.3\left(\mathrm{CH}_{2}\right), 32.8$ $\left(\mathrm{CH}_{2}\right), 23.3\left(\mathrm{CH}_{2}\right), 22.5\left(\mathrm{CH}_{2}\right), 22.0\left(\mathrm{CH}_{2}\right), 21.8\left(\mathrm{CH}_{2}\right), 21.17\left(\mathrm{CH}_{2}\right), 21.10$ $\left(\mathrm{CH}_{2}\right)$.

HRMS (ESI+): $m / z[\mathrm{M}+\mathrm{H}]^{+}$calcd for $\mathrm{C}_{23} \mathrm{H}_{26} \mathrm{NO}_{6}: 412.1755$; found: 412.1754.

10-Benzyl-4-(furan-2-yl)-8a-hydroxyhexahydro-2H,5H-2,4a-(epiminomethano)chromen-9-one (5nc)

Following the general procedure, the reaction of $N$-benzyl-2-oxocyclohexanecarboxamide (1n; $300 \mathrm{mg}, 1.29 \mathrm{mmol}$ ) with furylacrolein (2c; $101 \mu \mathrm{L}, 1.41 \mathrm{mmol}$ ) in the presence of PS-BEMP (59 mg, $10 \mathrm{~mol} \%$ ) in THF ( $3 \mathrm{~mL}$ ) heated at $55-60{ }^{\circ} \mathrm{C}$ for $24 \mathrm{~h}$ afforded the product $5 \mathrm{nc}$ as a white solid (401 mg, 80\%) with 10:1 dr. One diastereomer was separated.

First fraction: one diastereomer was isolated as a colorless oil (13\%); $R_{f}=0.43\left(\mathrm{Et}_{2} \mathrm{O} / \mathrm{PE}, 7: 3\right)$

${ }^{1} \mathrm{H} \mathrm{NMR}\left(300 \mathrm{MHz}, \mathrm{CDCl}_{3}\right): \delta=7.36(\mathrm{dd}, J=1.8,0.7 \mathrm{~Hz}, 1 \mathrm{H}), 7.31-7.25$ $(\mathrm{m}, 6 \mathrm{H}), 6.33(\mathrm{dd}, J=3.2,1.9 \mathrm{~Hz}, 1 \mathrm{H}), 6.22(\mathrm{~d}, J=3.2 \mathrm{~Hz}, 1 \mathrm{H}), 5.02$ $(\mathrm{dd}, J=3.1,1.5 \mathrm{~Hz}, 1 \mathrm{H}), 4.84(\mathrm{~d}, J=14.9 \mathrm{~Hz}, 1 \mathrm{H}), 4.38(\mathrm{~d}, J=14.9 \mathrm{~Hz}, 1$ 
H), $3.86(\mathrm{~d}, J=1.8 \mathrm{~Hz}, 1 \mathrm{H}), 3.07(\mathrm{dd}, J=10.8,8.6 \mathrm{~Hz}, 1 \mathrm{H}), 2.53(\mathrm{ddd}, J=$ 13.6, 8.6, $1.6 \mathrm{~Hz}, 1 \mathrm{H}), 2.05-1.97(\mathrm{~m}, 2 \mathrm{H}), 1.79(\mathrm{dd}, J=12.7,8.6 \mathrm{~Hz}, 1$ H), $1.56-1.45(\mathrm{~m}, 3 \mathrm{H}), 1.26-1.13(\mathrm{~m}, 2 \mathrm{H})$.

${ }^{13} \mathrm{C}$ NMR (75 MHz, $\left.\mathrm{CDCl}_{3}\right): \delta=173.0(\mathrm{C}), 151.8(\mathrm{C}), 142.1(\mathrm{CH}), 136.8$ (C), $128.9(2 \mathrm{CH}), 128.0(2 \mathrm{CH}), 127.8(\mathrm{CH}), 111.0(\mathrm{CH}), 110.2(\mathrm{CH})$, 99.6 (C), $80.7(\mathrm{C}-\mathrm{O}), 51.8\left(\mathrm{CH}_{2}\right), 47.4(\mathrm{C}), 38.9\left(\mathrm{CH}_{2}\right), 36.2(\mathrm{CH}), 33.5$ $\left(\mathrm{CH}_{2}\right), 26.1\left(\mathrm{CH}_{2}\right), 22.1\left(\mathrm{CH}_{2}\right), 21.7\left(\mathrm{CH}_{2}\right)$.

Second fraction: a mixture of two diastereomers was isolated (67\%); $R_{f}=0.16\left(\mathrm{Et}_{2} \mathrm{O} / \mathrm{PE}, 7: 3\right)$.

${ }^{1} \mathrm{H} \mathrm{NMR}\left(300 \mathrm{MHz}, \mathrm{CDCl}_{3}\right): \delta=7.42(\mathrm{dd}, J=11.8,1.6 \mathrm{~Hz}, 1 \mathrm{H}), 7.37(\mathrm{~d}$, $J=2.0 \mathrm{~Hz}, 1 \mathrm{H}), 7.34(\mathrm{t}, J=2.0 \mathrm{~Hz}, 3 \mathrm{H}), 7.31(\mathrm{~d}, J=1.9 \mathrm{~Hz}, 2 \mathrm{H}), 7.29$ $(\mathrm{dd}, J=4.0,2.0 \mathrm{~Hz}, 3 \mathrm{H}), 7.25(\mathrm{dd}, J=2.3,1.6 \mathrm{~Hz}, 2 \mathrm{H}), 7.20-7.17(\mathrm{~m}, 1$ H), $6.24(\mathrm{dd}, J=3.2,1.9 \mathrm{~Hz}, 1 \mathrm{H}), 6.20(\mathrm{dd}, J=3.2,1.9 \mathrm{~Hz}, 1 \mathrm{H}), 5.98(\mathrm{~d}$, $J=3.2 \mathrm{~Hz}, 1 \mathrm{H}), 5.88(\mathrm{~d}, J=3.1 \mathrm{~Hz}, 1 \mathrm{H}), 5.08-5.04(\mathrm{~m}, 1 \mathrm{H}), 4.98(\mathrm{t}, J=$ $2.3 \mathrm{~Hz}, 1 \mathrm{H}), 4.89(\mathrm{~s}, 1 \mathrm{H}), 4.84-4.77(\mathrm{~m}, 1 \mathrm{H}), 4.53(\mathrm{dd}, J=14.9,3.3 \mathrm{~Hz}$, $1 \mathrm{H}), 3.68(\mathrm{dd}, J=10.3,3.6 \mathrm{~Hz}, 1 \mathrm{H}), 3.58(\mathrm{dd}, J=10.6,4.8 \mathrm{~Hz}, 1 \mathrm{H}), 3.44$ (s, $1 \mathrm{H}), 2.81(\mathrm{~s}, 1 \mathrm{H}), 2.56$ (ddd, $J=12.6,10.3,2.1 \mathrm{~Hz}, 1 \mathrm{H}), 2.30$ (td, $J=$ $10.5,5.3 \mathrm{~Hz}, 1 \mathrm{H}), 2.10-1.70(\mathrm{~m}, 8 \mathrm{H}), 1.63-1.13(\mathrm{~m}, 10 \mathrm{H})$.

${ }^{13} \mathrm{C}$ NMR $\left(75 \mathrm{MHz}, \mathrm{CDCl}_{3}\right.$ ): $\delta$ (major): = 171.19 (C), 154.84 (C), 141.84 $(\mathrm{CH}), 136.86(\mathrm{C}), 128.66(2 \mathrm{CH}), 128.58(2 \mathrm{CH}), 127.69(\mathrm{CH}), 110.14$ $(\mathrm{CH}), 107.02(\mathrm{CH}), 98.14(\mathrm{C}), 80.97(\mathrm{CH}), 51.82(\mathrm{C}), 47.50\left(\mathrm{CH}_{2}\right), 38.52$ $\left(\mathrm{CH}_{2}\right), 34.90\left(\mathrm{CH}_{2}\right), 33.30(\mathrm{CH}), 26.83\left(\mathrm{CH}_{2}\right), 22.43\left(\mathrm{CH}_{2}\right), 21.92\left(\mathrm{CH}_{2}\right) ; \delta$ $($ minor $)=170.8(\mathrm{C}), 154.1(\mathrm{C}), 142.0(\mathrm{CH}), 136.9(\mathrm{C}), 128.7(2 \mathrm{CH})$, $128.04(\mathrm{CH}), 127.7(2 \mathrm{CH}), 110.2(\mathrm{CH}), 107.06(\mathrm{CH}), 93.3(\mathrm{C}), 80.9(\mathrm{CH})$, $51.2(\mathrm{C}), 47.8(\mathrm{CH}), 35.6\left(\mathrm{CH}_{2}\right), 33.4\left(\mathrm{CH}_{2}\right), 30.7\left(\mathrm{CH}_{2}\right), 23.5\left(\mathrm{CH}_{2}\right), 22.0$ $\left(\mathrm{CH}_{2}\right), 19.6\left(\mathrm{CH}_{2}\right)$.

HRMS (ESI+): $m / z[\mathrm{M}+\mathrm{H}]^{+}$calcd for $\mathrm{C}_{21} \mathrm{H}_{24} \mathrm{NO}_{4}: 354.1700$; found: 354.1694, 354.1700.

\section{Reactivity of Spirohemiaminals}

\section{Dehydration; General Procedure 1}

Under an argon atmosphere, Burgess reagent (1-2 equiv) was added to a $0.1 \mathrm{M}$ solution of spirohemiaminal compound (1 equiv) in THF. The mixture was then stirred at reflux for $2 \mathrm{~h}$. The solvent was removed under vacuum, the residue was washed with $\mathrm{H}_{2} \mathrm{O}$, and the organic layer was extracted with EtOAc $(2 \times)$. The combined organic phases were dried $\left(\mathrm{Na}_{2} \mathrm{SO}_{4}\right)$, filtered, and concentrated under vacuum to give the crude product. Finally, flash chromatography on silica gel afforded the desired product.

\section{7-Allyl-7-azaspiro[4.5]dec-8-ene-1,6-dione (6ia)}

Following the general procedure 1 for dehydration, the reaction of spirohemiaminal compund $\mathbf{3 b a}(210 \mathrm{mg}, 0.95 \mathrm{mmol})$ with Burgess reagent $(187 \mathrm{mg}, 0.98 \mathrm{mmol})$ in THF $(2.5 \mathrm{~mL})$ afforded the product $\mathbf{6 i a}$ as a yellow oil $(250 \mathrm{mg}, 64 \%)$. The crude product was obtained with $1: 1 \mathrm{dr} ; R_{f}=0.47\left(\mathrm{Et}_{2} \mathrm{O} / \mathrm{PE}, 7: 3\right)$. Data given are for the mixture of two diastereomers.

${ }^{1} \mathrm{H} \mathrm{NMR}\left(300 \mathrm{MHz}, \mathrm{CDCl}_{3}\right): \delta=6.02(\mathrm{~d}, J=2.2 \mathrm{~Hz}, 1 \mathrm{H}), 6.00(\mathrm{~d}, J=2.2$ $\mathrm{Hz}, 1 \mathrm{H}$ ), 5.75 (ddd, $J=15.9,10.7,5.6 \mathrm{~Hz}, 2 \mathrm{H}), 5.21-5.14(\mathrm{~m}, 4 \mathrm{H}), 5.10$ $(\mathrm{ddd}, J=10.8,5.5,3.4 \mathrm{~Hz}, 2 \mathrm{H}), 4.12(\mathrm{ddt}, J=15.6,5.5,1.5 \mathrm{~Hz}, 2 \mathrm{H}), 4.04$ (ddt, $J=15.6,5.6,1.5 \mathrm{~Hz}, 2 \mathrm{H}), 2.77-2.75(\mathrm{~m}, 1 \mathrm{H}), 2.73-2.71(\mathrm{~m}, 1 \mathrm{H})$, 2.55-2.39 (m, $4 \mathrm{H}), 2.35-2.24$ (m, $2 \mathrm{H}), 2.14-2.00$ ( $\mathrm{m}, 4 \mathrm{H}), 1.95-1.84$ $(\mathrm{m}, 4 \mathrm{H})$.

${ }^{13} \mathrm{C} \mathrm{NMR}\left(75 \mathrm{MHz}, \mathrm{CDCl}_{3}\right): \delta=216.3(2 \mathrm{C}), 169.1(2 \mathrm{C}), 132.6(2 \mathrm{CH})$, $128.8(2 \mathrm{CH}), 117.4\left(2 \mathrm{CH}_{2}\right), 103.7(2 \mathrm{C}), 55.0(2 \mathrm{C}), 48.1\left(2 \mathrm{CH}_{2}\right), 38.4$ $\left(2 \mathrm{CH}_{2}\right), 35.1\left(2 \mathrm{CH}_{2}\right), 29.4\left(2 \mathrm{CH}_{2}\right), 19.1\left(2 \mathrm{CH}_{2}\right)$.
Methyl 1,6-Dioxo-7,10-diphenyl-7-azaspiro[4.5]dec-8-ene-8-carboxylate $(6 \mathrm{fd})$

Following the general procedure 1 for dehydration, the reaction of spirohemiaminal compound 3fd (168 mg, $0.42 \mathrm{mmol}$ ) with Burgess reagent ( $203.7 \mathrm{mg}, 0.84 \mathrm{mmol}$ ) in THF ( $5 \mathrm{~mL}$ ) afforded the product $\mathbf{6 f d}$ as a yellow solid (114 mg, 72\%). The crude product was obtained with $1: 1 \mathrm{dr} ; R_{f}=0.56\left(\mathrm{Et}_{2} \mathrm{O} / \mathrm{PE}, 7: 3\right)$. Data given are for the mixture of two diastereomers.

${ }^{1} \mathrm{H}$ NMR (300 MHz, $\left.\mathrm{CDCl}_{3}\right): \delta=7.36-7.27(\mathrm{~m}, 10 \mathrm{H}), 7.22-7.18(\mathrm{~m}, 10$ $\mathrm{H}), 6.51(\mathrm{~d}, J=4.4 \mathrm{~Hz}, 1 \mathrm{H}), 6.45(\mathrm{~d}, J=3.5 \mathrm{~Hz}, 1 \mathrm{H}), 4.36(\mathrm{~d}, J=4.4 \mathrm{~Hz}$, $1 \mathrm{H}), 4.03(\mathrm{~d}, J=3.4 \mathrm{~Hz}, 1 \mathrm{H}), 3.49(\mathrm{~s}, 3 \mathrm{H}), 3.48(\mathrm{~s}, 3 \mathrm{H}), 2.83(\mathrm{dt}, J=$ 13.4, $6.8 \mathrm{~Hz}, 1 \mathrm{H}$ ), 2.26 (ddd, $J=16.4,13.6,6.5 \mathrm{~Hz}, 3 \mathrm{H}), 1.99-1.79(\mathrm{~m}$, $6 \mathrm{H}), 1.53$ (dd, $J=18.0,6.7 \mathrm{~Hz}, 1 \mathrm{H}), 1.24-1.12(\mathrm{~m}, 1 \mathrm{H})$.

${ }^{13} \mathrm{C}$ NMR (75 MHz, $\mathrm{CDCl}_{3}$ ): $\delta=213.5(\mathrm{C}), 213.18$ (C), 169.13 (C), 167.7 (C), 162.5 (C), 162.5 (C), 139.4 (C), 138.7 (C), 138.1 (C), 136.76 (C), $134.3(\mathrm{C}), 134.0(\mathrm{C}), 129.1(2 \mathrm{CH}), 129.08(2 \mathrm{CH}), 128.8(2 \mathrm{CH}), 128.7$ $(2 \mathrm{CH}), 128.6(2 \mathrm{CH}), 128.5(2 \mathrm{CH}), 128.0(\mathrm{CH}), 127.8(\mathrm{CH}), 127.2(\mathrm{CH})$, $127.1(\mathrm{CH}), 126.4(2 \mathrm{CH}), 126.2(2 \mathrm{CH}), 122.5(\mathrm{CH}), 122.1(\mathrm{CH}), 59.9$ (C), $59.14(\mathrm{C}), 52.2\left(\mathrm{CH}_{3}\right), 52.1\left(\mathrm{CH}_{3}\right), 47.8(\mathrm{CH}), 43.0(\mathrm{CH}), 39.8\left(\mathrm{CH}_{2}\right)$, $38.4\left(\mathrm{CH}_{2}\right), 31.8\left(\mathrm{CH}_{2}\right), 29.2\left(\mathrm{CH}_{2}\right), 19.1\left(\mathrm{CH}_{2}\right), 18.8\left(\mathrm{CH}_{2}\right)$.

\section{Methyl 3,3-Dimethyl-1,6-dioxo-10-phenyl-7-azaspiro[4.5]dec-8-} ene-8-carboxylate (6gd)

Following the general procedure 1 for dehydration, the reaction of spirohemiaminal compound 3gd (105 mg, $0.30 \mathrm{mmol}$ ) with Burgess reagent (145 $\mathrm{mg}, 0.60 \mathrm{mmol}$ ) in THF $(3.5 \mathrm{~mL})$ afforded the product 6gd as a white solid (95 mg, 96\%). The crude product was obtained with $1: 1 \mathrm{dr} ; R_{f}=0.51\left(\mathrm{Et}_{2} \mathrm{O} / \mathrm{PE}, 7: 3\right)$. Data given are for the mixture of two diastereomers.

${ }^{1} \mathrm{H}$ NMR $\left(300 \mathrm{MHz}, \mathrm{CDCl}_{3}\right): \delta=7.89(\mathrm{~s}, 1 \mathrm{H}), 7.83(\mathrm{~s}, 1 \mathrm{H}), 7.33-7.28$ $(\mathrm{m}, 5 \mathrm{H}), 7.20(\mathrm{~d}, J=2.4 \mathrm{~Hz}, 1 \mathrm{H}), 7.17(\mathrm{~d}, J=1.5 \mathrm{~Hz}, 1 \mathrm{H}), 7.14(\mathrm{~d}, J=1.9$ $\mathrm{Hz}, 1 \mathrm{H}), 7.12(\mathrm{~d}, J=2.4 \mathrm{~Hz}, 1 \mathrm{H}), 6.33(\mathrm{dd}, J=4.7,1.4 \mathrm{~Hz}, 1 \mathrm{H}), 6.25$ (dd, $J=2.6,1.6 \mathrm{~Hz}, 1 \mathrm{H}), 4.08(\mathrm{~d}, J=4.7 \mathrm{~Hz}, 1 \mathrm{H}), 3.81(\mathrm{~s}, 3 \mathrm{H}), 3.80$ (s, 3 $\mathrm{H}), 2.66(\mathrm{~d}, J=13.7 \mathrm{~Hz}, 1 \mathrm{H}), 2.36(\mathrm{~d}, J=14.8 \mathrm{~Hz}, 1 \mathrm{H}), 2.24(\mathrm{~d}, J=14.3$ $\mathrm{Hz}, 1 \mathrm{H}), 1.98-1.91$ (m, $2 \mathrm{H}), 1.90-1.75$ (m, $4 \mathrm{H}), 1.09$ (s, $4 \mathrm{H}), 1.02(\mathrm{~s}, 3$ $\mathrm{H}), 0.70(\mathrm{~s}, 3 \mathrm{H}), 0.69(\mathrm{~s}, 3 \mathrm{H})$.

${ }^{13} \mathrm{C} \mathrm{NMR}\left(75 \mathrm{MHz}, \mathrm{CDCl}_{3}\right.$ ): $\delta=213.2(\mathrm{C}), 212.2(\mathrm{C}), 169.2(\mathrm{C}), 168.1(\mathrm{C})$, $162.0(\mathrm{C}), 162.0(\mathrm{C}), 138.0(2 \mathrm{C}), 129.9(2 \mathrm{CH}), 129.1(2 \mathrm{CH}), 128.9(2$ $\mathrm{CH}), 128.7(2 \mathrm{CH}), 128.3(\mathrm{CH}), 128.2(\mathrm{CH}), 127.9(\mathrm{C}), 127.1(\mathrm{C}), 117.1$ $(\mathrm{CH}), 116.5(\mathrm{CH}), 60.2(\mathrm{C}), 60.0(\mathrm{C}), 54.8\left(\mathrm{CH}_{2}\right), 53.5\left(\mathrm{CH}_{2}\right), 52.7\left(\mathrm{CH}_{3}\right)$, $52.7\left(\mathrm{CH}_{3}\right), 49.7(\mathrm{CH}), 46.7(\mathrm{CH}), 43.9\left(\mathrm{CH}_{2}\right), 42.3\left(\mathrm{CH}_{2}\right), 32.8(\mathrm{C}), 32.5$ (C), $29.9\left(\mathrm{CH}_{3}\right), 29.7\left(\mathrm{CH}_{3}\right), 29.6\left(\mathrm{CH}_{3}\right), 29.4\left(\mathrm{CH}_{3}\right)$.

\section{Nucleophilic Substitution; General Procedure 2}

A solution of the corresponding spiro compound in anhyd $\mathrm{CH}_{2} \mathrm{Cl}_{2}(6$ $\mathrm{mL} / \mathrm{mmol}$ ) was cooled to $-78^{\circ} \mathrm{C}$, and then the corresponding silane (4 equiv) and the Lewis acid ( 1 equiv) were added via syringe. After stirring for $3 \mathrm{~h}$ at $-78{ }^{\circ} \mathrm{C}$ and $1 \mathrm{~h}$ at r.t., sat. aq $\mathrm{NaHCO}_{3}(10 \mathrm{~mL} / 1 \mathrm{mmol})$ was added. The mixture was extracted with $\mathrm{CH}_{2} \mathrm{Cl}_{2}$ and the organic layer was dried $\left(\mathrm{Na}_{2} \mathrm{SO}_{4}\right)$, filtered, and concentrated under vacuum to give the crude product. Finally, flash chromatography on silica gel afforded the desired product.

\section{7,8-Aiallyl-7-azaspiro[4.5]decane-1,6-dione (8baa)}

Following the general procedure 2 for substitution, the reaction of spirohemiaminal compound 3ba $(140 \mathrm{mg}, 0.62 \mathrm{mmol})$ with allyltrimethylsilane $(405 \mu \mathrm{L}, 2.51 \mathrm{mmol})$ and $\mathrm{BF}_{3} \cdot \mathrm{OEt}_{2}(77.5 \mu \mathrm{L}, 0.62$ $\mathrm{mmol}$ ) in $\mathrm{CH}_{2} \mathrm{Cl}_{2}(4 \mathrm{~mL}$ ) afforded the product 8 baa as a yellow oil (155 $\mathrm{mg}, 72 \%$ ). The crude product was obtained with $1: 1 \mathrm{dr} ; R_{f}=0.55$ $\left(\mathrm{Et}_{2} \mathrm{O} / \mathrm{PE}, 9: 1\right)$. Data given are for the mixture of two diastereomers. 
${ }^{1} \mathrm{H} \mathrm{NMR}\left(300 \mathrm{MHz}, \mathrm{CDCl}_{3}\right): \delta=5.76-5.56(\mathrm{~m}, 4 \mathrm{H}), 5.10-5.03(\mathrm{~m}, 8 \mathrm{H})$, $4.50-4.41(\mathrm{~m}, 1 \mathrm{H}), 4.36$ (ddt, $J=15.5,4.4,1.6 \mathrm{~Hz}, 1 \mathrm{H}), 3.52-3.41(\mathrm{~m}$, $2 \mathrm{H}), 3.39-3.31(\mathrm{~m}, 1 \mathrm{H}), 2.50-2.03(\mathrm{~m}, 8 \mathrm{H}), 2.19-2.03(\mathrm{~m}, 6 \mathrm{H}), 1.91$ $1.63(\mathrm{~m}, 10 \mathrm{H}), 1.46-1.39(\mathrm{~m}, 1 \mathrm{H})$.

$\left.{ }^{13} \mathrm{C} \mathrm{NMR} \mathrm{(75} \mathrm{MHz,} \mathrm{CDCl}_{3}\right)$ : $\delta=218.1(\mathrm{C}), 217.1(\mathrm{C}), 170.5(\mathrm{C}), 170.1(\mathrm{C})$ $134.1(\mathrm{CH}), 133.7(\mathrm{CH}), 133.1(\mathrm{CH}), 132.7(\mathrm{CH}), 118.2\left(\mathrm{CH}_{2}\right), 118.1$ $\left(\mathrm{CH}_{2}\right), 116.5\left(\mathrm{CH}_{2}\right), 116.5\left(\mathrm{CH}_{2}\right), 56.4(\mathrm{CH}), 55.5(\mathrm{CH}), 55.4(\mathrm{C}), 55.2(\mathrm{C})$ 47.8 $\left(\mathrm{CH}_{2}\right), 47.6\left(\mathrm{CH}_{2}\right), 38.9\left(\mathrm{CH}_{2}\right), 37.8\left(\mathrm{CH}_{2}\right), 37.3\left(\mathrm{CH}_{2}\right), 37.2\left(\mathrm{CH}_{2}\right)$, 36.7 $\left(\mathrm{CH}_{2}\right), 36.6\left(\mathrm{CH}_{2}\right), 26.4\left(\mathrm{CH}_{2}\right), 25.9\left(\mathrm{CH}_{2}\right), 22.5\left(\mathrm{CH}_{2}\right), 22.1\left(\mathrm{CH}_{2}\right)$, $19.7\left(\mathrm{CH}_{2}\right), 19.6\left(\mathrm{CH}_{2}\right)$.

HRMS (ESI+): $m / z[\mathrm{M}+\mathrm{H}]^{+}$calcd for $\mathrm{C}_{15} \mathrm{H}_{22} \mathrm{NO}_{2}: 248.1645$; found: $248.1640,248.1642$.

8-Allyl-7-(but-3-en-1-yl)-7-azaspiro[4.5]decane-1,6-dione (8caa)

Following the general procedure 2 for substitution, the reaction of spirohemiaminal compound $\mathbf{3 c a}(170 \mathrm{mg}, 0.76 \mathrm{mmol})$ with allyltrimethylsilane ( $495 \mu \mathrm{L}, 3.07 \mathrm{mmol}$ ) and $\mathrm{BF}_{3} \cdot \mathrm{OEt}_{2}(95 \mu \mathrm{L}, 0.76 \mathrm{mmol}$ ) in $\mathrm{CH}_{2} \mathrm{Cl}_{2}(5 \mathrm{~mL})$ afforded the product 8 caa as a white solid $(155 \mathrm{mg}$, $78 \%)$. The crude product was obtained with 1:1 dr; $R_{f}=0.20\left(\mathrm{Et}_{2} \mathrm{O} / \mathrm{PE}\right.$, 9:1). Data given are for the mixture of two diastereomers.

${ }^{1} \mathrm{H} \mathrm{NMR}\left(300 \mathrm{MHz}, \mathrm{CDCl}_{3}\right): \delta=5.77-5.53(\mathrm{~m}, 4 \mathrm{H}), 5.08-4.91(\mathrm{~m}, 8 \mathrm{H})$, $3.85-3.76(\mathrm{~m}, 2 \mathrm{H}), 3.39(\mathrm{dt}, J=9.1,4.3 \mathrm{~Hz}, 1 \mathrm{H}), 3.30(\mathrm{dt}, J=9.3,7.3$ $\mathrm{Hz}, 1 \mathrm{H}), 2.83-2.72$ (m, $2 \mathrm{H}), 2.41-2.27$ (m, $8 \mathrm{H}), 2.20-2.07$ (m, $8 \mathrm{H})$, $1.85-1.73(\mathrm{~m}, 10 \mathrm{H}), 1.41-1.34(\mathrm{~m}, 2 \mathrm{H})$.

$\left.{ }^{13} \mathrm{C} \mathrm{NMR} \mathrm{(75} \mathrm{MHz,} \mathrm{CDCl}\right)$ ): $\delta=218.3(\mathrm{C}), 217.0(\mathrm{C}), 170.6(\mathrm{C}), 170.0(\mathrm{C})$ $135.2(\mathrm{CH}), 135.0(\mathrm{CH}), 134.0(\mathrm{CH}), 133.7(\mathrm{CH}), 118.2\left(\mathrm{CH}_{2}\right), 118.1$ $\left(\mathrm{CH}_{2}\right), 116.7\left(\mathrm{CH}_{2}\right), 116.5\left(\mathrm{CH}_{2}\right), 56.3(2 \mathrm{CH}), 56.29(\mathrm{C}), 55.09(\mathrm{C}), 45.5$ $\left(\mathrm{CH}_{2}\right), 45.2\left(\mathrm{CH}_{2}\right), 38.9\left(\mathrm{CH}_{2}\right), 37.7\left(\mathrm{CH}_{2}\right), 37.5\left(\mathrm{CH}_{2}\right), 37.4\left(\mathrm{CH}_{2}\right), 36.9$ $\left(\mathrm{CH}_{2}\right), 36.5\left(\mathrm{CH}_{2}\right), 31.9\left(\mathrm{CH}_{2}\right), 31.8\left(\mathrm{CH}_{2}\right), 26.4\left(\mathrm{CH}_{2}\right), 25.7\left(\mathrm{CH}_{2}\right), 22.3$ $\left(\mathrm{CH}_{2}\right), 22.1\left(\mathrm{CH}_{2}\right), 19.7\left(\mathrm{CH}_{2}\right), 19.5\left(\mathrm{CH}_{2}\right)$.

\section{8-Allyl-7-(2-bromobenzyl)-7-azaspiro[4.5]decane-1,6-dione (8daa)}

Following the general procedure 2 for substitution, the reaction of spirohemiaminal compound 3da $(208 \mathrm{mg}, 0.59 \mathrm{mmol})$ with allyltrimethylsilane ( $382 \mu \mathrm{L}, 2.37 \mathrm{mmol}$ ) and $\mathrm{BF}_{3} \cdot \mathrm{OEt}_{2}(74 \mu \mathrm{L}, 0.59 \mathrm{mmol})$ in $\mathrm{CH}_{2} \mathrm{Cl}_{2}(5 \mathrm{~mL})$ afforded the product 8daa as a yellow solid $(205 \mathrm{mg}$, $92 \%)$. The crude product was obtained with $1: 1 \mathrm{dr} ; R_{f}=0.40\left(\mathrm{Et}_{2} \mathrm{O} / \mathrm{PE}\right.$ 7:3). Data given are for the mixture of two diastereomers.

${ }^{1} \mathrm{H}$ NMR $\left(300 \mathrm{MHz}, \mathrm{CDCl}_{3}\right): \delta=7.47(\mathrm{dt}, J=7.9,1.5 \mathrm{~Hz}, 2 \mathrm{H}), 7.32(\mathrm{ddd}$, $J=9.2,7.4,1.4 \mathrm{~Hz}, 2 \mathrm{H}), 7.18$ (ddd, $J=9.4,7.5,1.4 \mathrm{~Hz}, 2 \mathrm{H}), 7.06$ (td, $J=$ $7.9,1.4 \mathrm{~Hz}, 2 \mathrm{H}), 5.68-5.51(\mathrm{~m}, 2 \mathrm{H}), 5.23(\mathrm{~d}, J=16.3 \mathrm{~Hz}, 1 \mathrm{H}), 5.12$ $5.02(\mathrm{~m}, 4 \mathrm{H}), 4.96(\mathrm{~d}, J=16.0 \mathrm{~Hz}, 1 \mathrm{H}), 4.35(\mathrm{~d}, J=16.0 \mathrm{~Hz}, 1 \mathrm{H}), 4.19$ $(\mathrm{d}, J=16.3 \mathrm{~Hz}, 1 \mathrm{H}), 3.36-3.27(\mathrm{~m}, 2 \mathrm{H}), 2.76-2.44(\mathrm{~m}, 4 \mathrm{H}), 2.36-2.11$ $(\mathrm{m}, 9 \mathrm{H}), 1.93-1.71(\mathrm{~m}, 10 \mathrm{H}), 1.53-1.45(\mathrm{~m}, 1 \mathrm{H})$.

${ }^{13} \mathrm{C}$ NMR (75 MHz, $\mathrm{CDCl}_{3}$ ): $\delta=217.97(\mathrm{C}), 217.09$ (C), $171.01(\mathrm{C})$, $170.54(\mathrm{C}), 136.19$ (C), $135.61(\mathrm{C}), 133.88(\mathrm{CH}), 133.60(\mathrm{CH}), 132.64$ $(\mathrm{CH}), 132.48(\mathrm{CH}), 128.62(\mathrm{CH}), 128.59(\mathrm{CH}), 128.51(\mathrm{CH}), 128.12$ $(\mathrm{CH}), 127.89(\mathrm{CH}), 127.65(\mathrm{CH}), 123.12(\mathrm{C}), 123.11(\mathrm{C}), 118.46\left(\mathrm{CH}_{2}\right)$, $118.30\left(\mathrm{CH}_{2}\right), 56.54(\mathrm{CH}), 56.24(\mathrm{CH}), 55.55(\mathrm{C}), 55.32(\mathrm{C}), 48.72\left(\mathrm{CH}_{2}\right)$, $48.04\left(\mathrm{CH}_{2}\right), 38.80\left(\mathrm{CH}_{2}\right), 37.66\left(\mathrm{CH}_{2}\right), 37.16\left(\mathrm{CH}_{2}\right), 37.11\left(\mathrm{CH}_{2}\right), 36.89$ $\left(\mathrm{CH}_{2}\right), 36.56\left(\mathrm{CH}_{2}\right), 26.35\left(\mathrm{CH}_{2}\right), 25.97\left(\mathrm{CH}_{2}\right), 22.57\left(\mathrm{CH}_{2}\right), 21.91\left(\mathrm{CH}_{2}\right)$, $19.77\left(\mathrm{CH}_{2}\right), 19.62\left(\mathrm{CH}_{2}\right)$.

HRMS (ESI+): $m / z[\mathrm{M}+\mathrm{H}]^{+}$calc for $\mathrm{C}_{19} \mathrm{H}_{22} \mathrm{BrNO}_{2}$ : 376.0907; found: 376.0919, 376.0917.

\section{1,6-Dioxo-7-phenyl-7-azaspiro[4.5]decane-8-carbonitrile (8fab)} Following the general procedure 2 for substitution, the reaction of spirohemiaminal $\mathbf{3 f a}(260 \mathrm{mg}, 1.28 \mathrm{mmol})$ with trimethylsilyl cyanide $(382.55 \mu \mathrm{L}, 5.12 \mathrm{mmol})$ and $\mathrm{BF}_{3} \cdot \mathrm{OEt}_{2}(158 \mu \mathrm{L}, 1.28 \mathrm{mmol})$ in $\mathrm{CH}_{2} \mathrm{Cl}_{2}(9 \mathrm{~mL})$ afforded the product 8 fab as a white solid $(152 \mathrm{mg}$. $44 \%) ; R_{f}=0.23\left(\mathrm{Et}_{2} \mathrm{O} / \mathrm{PE}, 7: 3\right)$ as a unique diastereomer that partially crystallized as white needles for X-ray analysis; $\mathrm{mp} 168^{\circ} \mathrm{C}$.

${ }^{1} \mathrm{H} \mathrm{NMR}\left(300 \mathrm{MHz}, \mathrm{CDCl}_{3}\right): \delta=7.44-7.37(\mathrm{~m}, 2 \mathrm{H}), 7.31-7.27(\mathrm{~m}, 3 \mathrm{H})$, $5.53(\mathrm{~d}, J=2.1 \mathrm{~Hz}, 1 \mathrm{H}), 2.80-2.71(\mathrm{~m}, 1 \mathrm{H}), 2.57-2.33(\mathrm{~m}, 3 \mathrm{H}), 2.21-$ $1.87(\mathrm{~m}, 4 \mathrm{H}), 1.80-1.58(\mathrm{~m}, 2 \mathrm{H})$.

${ }^{13} \mathrm{C} \mathrm{NMR}\left(75 \mathrm{MHz}, \mathrm{CDCl}_{3}\right): \delta=216.22(\mathrm{C}), 169.35$ (C), $141.15(\mathrm{C})$, $129.85(2 \mathrm{CH}), 128.55(\mathrm{CH}), 127.15(2 \mathrm{CH}), 118.18(\mathrm{C}), 55.58(\mathrm{C}), 52.71$ (CH), $37.77\left(\mathrm{CH}_{2}\right), 36.30\left(\mathrm{CH}_{2}\right), 28.01\left(\mathrm{CH}_{2}\right), 23.71\left(\mathrm{CH}_{2}\right), 19.68\left(\mathrm{CH}_{2}\right)$.

\section{7-Allyl-8-(propa-1,2-dien-1-yl)-7-azaspiro[4.5]decane-1,6-dione (8fac)}

Following the general procedure 2 for substitution, the reaction of spirohemiaminal compound $\mathbf{3 f a}(200 \mathrm{mg}, 0.89 \mathrm{mmol})$ with propargyltrimethylsilane $(401.55 \mu \mathrm{L}, 2.68 \mathrm{mmol})$ and $\mathrm{BF}_{3} \cdot \mathrm{OEt}_{2}(110.60 \mu \mathrm{L}$ $0.89 \mathrm{mmol})$ in $\mathrm{CH}_{2} \mathrm{Cl}_{2}(8 \mathrm{~mL})$ afforded the product 8 fac as a white oil (78 mg, 35\%); $R_{f}=0.23\left(\mathrm{Et}_{2} \mathrm{O} / \mathrm{PE}, 7: 3\right)$. Only a single isomer was isolated.

${ }^{1} \mathrm{H} \mathrm{NMR}\left(300 \mathrm{MHz}, \mathrm{CDCl}_{3}\right): \delta=5.80-5.67(\mathrm{~m}, 1 \mathrm{H}), 5.16-5.04(\mathrm{~m}, 2 \mathrm{H})$, $4.83(\mathrm{~d}, J=5.9 \mathrm{~Hz}, 1 \mathrm{H}), 4.53(\mathrm{dd}, J=15.0,3.7 \mathrm{~Hz}, 1 \mathrm{H}), 4.00(\mathrm{~d}, J=5.9$ $\mathrm{Hz}, 1 \mathrm{H}), 3.43(\mathrm{dd}, J=11.5,3.6 \mathrm{~Hz}, 1 \mathrm{H}), 2.99(\mathrm{~d}, J=17.4 \mathrm{~Hz}, 1 \mathrm{H}), 2.70$ $(\mathrm{d}, J=17.4 \mathrm{~Hz}, 1 \mathrm{H}), 2.27-2.11(\mathrm{~m}, 4 \mathrm{H}), 2.01-1.66(\mathrm{~m}, 6 \mathrm{H})$.

${ }^{13} \mathrm{C}$ NMR $\left(75 \mathrm{MHz}, \mathrm{CDCl}_{3}\right): \delta=212.3$ (C), 208.4 (C), $174.1(\mathrm{C}), 133.6$ $\left(\mathrm{CH}_{2}\right), 117.1\left(\mathrm{CH}_{2}\right), 92.3(\mathrm{CH}), 84.3\left(\mathrm{CH}_{2}\right), 54.5(\mathrm{C}), 48.9\left(\mathrm{CH}_{2}\right), 47.9$ $\left(\mathrm{CH}_{2}\right), 40.5\left(\mathrm{CH}_{2}\right), 39.1\left(\mathrm{CH}_{2}\right), 31.4(\mathrm{CH}), 27.3\left(\mathrm{CH}_{2}\right), 26.9\left(\mathrm{CH}_{2}\right)$.

1',6'-Diallylspiro[indene-2,3'-piperidine]-1,2'(3H)-dione (8iaa) Following the general procedure 2 for substitution, the reaction of spirohemiaminal compound 3ia $(140 \mathrm{mg}, 0.527 \mathrm{mmol})$ with allyltrimethylsilane ( $330 \mu \mathrm{L}, 2.1 \mathrm{mmol})$ and $\mathrm{BF}_{3} \cdot \mathrm{OEt}_{2}(65 \mu \mathrm{L}, 0,527 \mathrm{mmol})$ in $\mathrm{CH}_{2} \mathrm{Cl}_{2}(6 \mathrm{~mL})$ afforded the product 8iaa as a white oil $(132.6 \mathrm{mg}$, $87 \%)$. The crude product was obtained with $1: 1 \mathrm{dr} ; R_{f}\left(\mathrm{Et}_{2} \mathrm{O} / \mathrm{PE}, 7: 3\right)=$ 0.28 . Data given are for the mixture of two diastereomers.

${ }^{1} \mathrm{H}$ NMR $\left(300 \mathrm{MHz}, \mathrm{CDCl}_{3}\right.$ ): $\delta=7.74(\mathrm{dd}, J=11.4,7.7 \mathrm{~Hz}, 2 \mathrm{H}), 7.57(\mathrm{t}$ $J=7.4 \mathrm{~Hz}, 2 \mathrm{H}), 7.43(\mathrm{~d}, J=7.7 \mathrm{~Hz}, 2 \mathrm{H}), 7.36(\mathrm{dt}, J=11.6,5.8 \mathrm{~Hz}, 2 \mathrm{H})$, 5.87-5.63 (m, $4 \mathrm{H}), 5.31(\mathrm{~d}, J=17.2 \mathrm{~Hz}, 1 \mathrm{H}), 5.21-5.09(\mathrm{~m}, 6 \mathrm{H}), 4.65-$ $4.56(\mathrm{~m}, 1 \mathrm{H}), 4.49$ (dd, $J=15.5,4.6 \mathrm{~Hz}, 1 \mathrm{H}), 3.86(\mathrm{~d}, J=16.8 \mathrm{~Hz}, 1 \mathrm{H})$, 3.60 (ddd, $J=22.0,20.2,11.6 \mathrm{~Hz}, 5 \mathrm{H}$ ), 3.02 (d, $J=16.8 \mathrm{~Hz}, 1 \mathrm{H}$ ), 2.91 $(\mathrm{d}, J=16.8 \mathrm{~Hz}, 1 \mathrm{H}), 2.67-2.41(\mathrm{~m}, 5 \mathrm{H}), 2.33(\mathrm{dq}, J=13.0,4.1 \mathrm{~Hz}, 2 \mathrm{H})$, $2.01(\mathrm{dd}, J=7.2,5.2 \mathrm{~Hz}, 3 \mathrm{H}), 1.83-1.69(\mathrm{~m}, 2 \mathrm{H}), 1.66-1.56(\mathrm{~m}, 1 \mathrm{H})$.

${ }^{13} \mathrm{C} \mathrm{NMR}\left(75 \mathrm{MHz}, \mathrm{CDCl}_{3}\right): \delta$ (major) $=205.30(\mathrm{C}), 169.72(\mathrm{C}), 153.62$ (C), $135.77(\mathrm{C}), 134.69(\mathrm{CH}), 134.01(\mathrm{CH}), 133.01(\mathrm{CH}), 127.63(\mathrm{CH})$ $126.35(\mathrm{CH}), 124.80(\mathrm{CH}), 118.39\left(\mathrm{CH}_{2}\right), 116.81\left(\mathrm{CH}_{2}\right), 56.99(\mathrm{C}), 55.93$ $(\mathrm{CH}), 47.92\left(\mathrm{CH}_{2}\right), 41.12\left(\mathrm{CH}_{2}\right), 37.50\left(\mathrm{CH}_{2}\right), 28.00\left(\mathrm{CH}_{2}\right), 22.16\left(\mathrm{CH}_{2}\right) ; \delta$ $($ minor $)=205.71(\mathrm{C}), 170.10(\mathrm{C}), 153.76(\mathrm{C}), 135.12(\mathrm{C}), 134.25(\mathrm{CH})$ $133.18(\mathrm{CH}), 127.68(\mathrm{CH}), 126.48(\mathrm{CH}), 124.97(\mathrm{CH}), 118.49\left(\mathrm{CH}_{2}\right)$, $116.97\left(\mathrm{CH}_{2}\right), 57.03(\mathrm{C}), 56.07(\mathrm{CH}), 48.12\left(\mathrm{CH}_{2}\right), 42.52\left(\mathrm{CH}_{2}\right), 37.04$ $\left(\mathrm{CH}_{2}\right), 30.40(\mathrm{CH}), 28.14\left(\mathrm{CH}_{2}\right), 23.21\left(\mathrm{CH}_{2}\right)$.

1',6'-Diallyl-3,4-dihydro-1H-spiro[naphthalene-2,3'-piperidine]1,2'-dione (8jaa)

Following the general procedure 2 for substitution, the reaction of spirohemiaminal compound 3ka (100 mg, $0.35 \mathrm{mmol}$ ) with allyltrimethylsilane $(204.85 \mu \mathrm{L}, 1.4 \mathrm{mmol})$ and $\mathrm{BF}_{3} \cdot \mathrm{OEt}_{2}(37 \mu \mathrm{L}, 0,35$ $\mathrm{mmol})$ in $\mathrm{CH}_{2} \mathrm{Cl}_{2}(4 \mathrm{~mL})$ afforded the product 8 jaa as a white solid (65 
$\mathrm{mg}, 60 \%)$. The crude product was obtained with $1: 1 \mathrm{dr} ; R_{f}=0.34$ $\left(\mathrm{Et}_{2} \mathrm{O} / \mathrm{PE}, 7: 3\right)$ for the first diastereomer; $R_{f}=0.27\left(\mathrm{Et}_{2} \mathrm{O} / \mathrm{PE}, 7: 3\right)$ for the second diastereomer. Data given are for the mixture of two diastereomers.

${ }^{1} \mathrm{H}$ NMR $\left(300 \mathrm{MHz}, \mathrm{CDCl}_{3}\right): \delta=7.98(\mathrm{~d}, J=7.8 \mathrm{~Hz}, 2 \mathrm{H}), 7.40(\mathrm{t}, J=7.4$ $\mathrm{Hz}, 2 \mathrm{H}), 7.22(\mathrm{~d}, J=9.7 \mathrm{~Hz}, 2 \mathrm{H}), 7.16(\mathrm{~d}, J=7.6 \mathrm{~Hz}, 2 \mathrm{H}), 5.73$ (dtdd, $J=$ 24.1, 10.0, 7.3, $5.3 \mathrm{~Hz}, 4 \mathrm{H}$ ), 5.32 (d, $J=17.2 \mathrm{~Hz}, 1 \mathrm{H}), 5.19-5.03(\mathrm{~m}, 7$ $\mathrm{H}), 4.62-4.54(\mathrm{~m}, 1 \mathrm{H}), 4.43(\mathrm{dd}, J=15.6,4.5 \mathrm{~Hz}, 1 \mathrm{H}), 3.62(\mathrm{dd}, J=$ 15.7, $6.5 \mathrm{~Hz}, 1 \mathrm{H}), 3.54-3.43(\mathrm{~m}, 2 \mathrm{H}), 3.38(\mathrm{dt}, J=9.2,4.6 \mathrm{~Hz}, 1 \mathrm{H})$, $3.02-2.92(\mathrm{~m}, 4 \mathrm{H}), 2.81$ (ddd, $J=13.7,9.1,6.4 \mathrm{~Hz}, 1 \mathrm{H}), 2.56-2.16(\mathrm{~m}$, $5 \mathrm{H}), 2.06-1.88(\mathrm{~m}, 6 \mathrm{H}), 1.83-1.66(\mathrm{~m}, 4 \mathrm{H})$.

${ }^{13} \mathrm{C}$ NMR (75 MHz, $\mathrm{CDCl}_{3}$ ): $\delta$ (major) $=197.22(\mathrm{C}), 170.20(\mathrm{C}), 143.12$ (C), $134.27(\mathrm{CH}), 133.61(\mathrm{CH}), 133.07(\mathrm{CH}), 130.61(\mathrm{C}), 128.47(\mathrm{CH})$ $126.84(\mathrm{CH}), 118.31\left(\mathrm{CH}_{2}\right), 116.74\left(\mathrm{CH}_{2}\right), 55.25(\mathrm{C}), 53.40(\mathrm{CH}), 48.38$ $\left(\mathrm{CH}_{2}\right), 37.19\left(\mathrm{CH}_{2}\right), 33.41\left(\mathrm{CH}_{2}\right), 29.81(\mathrm{CH}), 25.14\left(\mathrm{CH}_{2}\right), 24.87\left(\mathrm{CH}_{2}\right)$ $21.06\left(\mathrm{CH}_{2}\right) ; \delta$ (minor) $=198.17(\mathrm{C}), 170.59(\mathrm{C}), 143.37(\mathrm{C}), 134.19$ $(\mathrm{CH}), 133.55(\mathrm{CH}), 131.68(\mathrm{C}), 128.31(\mathrm{CH}), 128.69(\mathrm{CH}), 126.92(\mathrm{CH})$ $118.38\left(\mathrm{CH}_{2}\right), 116.83\left(\mathrm{CH}_{2}\right), 55.90(\mathrm{C}), 54.15(\mathrm{CH}), 47.83\left(\mathrm{CH}_{2}\right), 37.49$ $\left(\mathrm{CH}_{2}\right), 32.32\left(\mathrm{CH}_{2}\right), 30.42(\mathrm{CH}), 25.17\left(\mathrm{CH}_{2}\right), 25.00\left(\mathrm{CH}_{2}\right), 22.51\left(\mathrm{CH}_{2}\right)$.

\section{Metathesis Cyclization; General Procedure 3}

To a one-necked round-bottomed flask, equipped with magnetic stirring bar under an argon atmosphere, were introduced spiro compound ( 1 equiv) and then the Grubbs catalyst ( $5 \mathrm{~mol} \%$ ). The mixture was stirred at r.t. for $4 \mathrm{~h}$ (the reaction was monitored by TLC). The mixture was then filtered and purified by flash chromatography on silica gel to separate the diastereomers.

\section{1',6',9',9a'-Tetrahydro-2'H,4'H-spiro[cyclopentane-1,3'-quino- lizine]-2,4'-dione (9baa)}

To a one-necked round-bottomed flask, equipped with a magnetic stirring bar under an argon atmosphere, were introduced spiro compound 8baa (370 $\mathrm{mg}, 1.49 \mathrm{mmol}$ ) in $\mathrm{CH}_{2} \mathrm{Cl}_{2}(46 \mathrm{~mL}$ ), followed by Grubbs II catalyst ( $64 \mathrm{mg}, 5 \mathrm{~mol} \%$ ). The mixture was stirred at r.t. for 4 $\mathrm{h}$ (the reaction was monitored by TLC). The mixture was then filtered and purified by flash chromatography on silica gel. The two diastereomers were separated.

First fraction: one diastereomer was isolated as a brown solid $(72 \mathrm{mg}$ $32 \%) ; \mathrm{mp} 106{ }^{\circ} \mathrm{C} ; R_{f}=0.23\left(\mathrm{Et}_{2} \mathrm{O} / \mathrm{PE}, 7: 3\right)$.

${ }^{1} \mathrm{H}$ NMR $\left(300 \mathrm{MHz}, \mathrm{CDCl}_{3}\right): \delta=5.75(\mathrm{ddd}, J=11.7,2.7,1.5 \mathrm{~Hz}, 1 \mathrm{H}$ ), 5.67 (ddd, $J=4.2,2.2,1.0 \mathrm{~Hz}, 1 \mathrm{H}), 4.59$ (dd, $J=19.2,2.4 \mathrm{~Hz}, 1 \mathrm{H}), 3.57$ $(\mathrm{dd}, J=14.6,7.8 \mathrm{~Hz}, 1 \mathrm{H}), 3.48(\mathrm{dd}, J=17.9,1.1 \mathrm{~Hz}, 1 \mathrm{H}), 2.58(\mathrm{dd}, J=$ 11.8, 7.0 Hz, $1 \mathrm{H}$ ), 2.54-2.43 (m, $1 \mathrm{H}$ ), 2.35-2.09 (m, $5 \mathrm{H}), 2.06-1.84$ (m, $3 \mathrm{H}), 1.62-1.49$ (dddd, $J=17.4,10.9,7.3,3.2 \mathrm{~Hz}, 2 \mathrm{H}$ ).

${ }^{13} \mathrm{C}$ NMR $\left(75 \mathrm{MHz}, \mathrm{CDCl}_{3}\right): \delta=217.69(\mathrm{C}), 170.06(\mathrm{C}), 124.23(\mathrm{CH})$ $123.75(\mathrm{CH}), 56.0(\mathrm{C}), 52.9(\mathrm{CH}), 42.5\left(\mathrm{CH}_{2}\right), 38.9\left(\mathrm{CH}_{2}\right), 36.5\left(\mathrm{CH}_{2}\right)$, $32.9\left(\mathrm{CH}_{2}\right), 27.3\left(\mathrm{CH}_{2}\right), 25.6\left(\mathrm{CH}_{2}\right), 19.5\left(\mathrm{CH}_{2}\right)$.

Second fraction: one diastereomer was isolated as a brown oil $(84 \mathrm{mg}$, $37 \%) ; R_{f}=0.18\left(\mathrm{Et}_{2} \mathrm{O} / \mathrm{PE}, 7: 3\right)$.

${ }^{1} \mathrm{H} \mathrm{NMR}\left(400 \mathrm{MHz}, \mathrm{CDCl}_{3}\right): \delta=5.78(\mathrm{ddd}, J=10.0,5.8,2.1 \mathrm{~Hz}, 1 \mathrm{H}$ ), 5.66 (ddd, $J=5.3,3.9,2.6 \mathrm{~Hz}, 1 \mathrm{H}$ ), 4.69 (dd, $J=18.7,3.0 \mathrm{~Hz}, 1 \mathrm{H}$ ), 3.55 (dt, $J=10.4,4.3 \mathrm{~Hz}, 1 \mathrm{H}$ ), 3.43 (ddd, $J=18.8,4.0,2.2 \mathrm{~Hz}, 1 \mathrm{H}$ ), 2.67$2.62(\mathrm{~m}, 1 \mathrm{H}), 2.53-2.44(\mathrm{~m}, 1 \mathrm{H}), 2.40-2.28(\mathrm{~m}, 2 \mathrm{H}), 2.22-2.16(\mathrm{~m}, 1$ H), 2.15-2.09 (m, $1 \mathrm{H}), 2.06$ (dd, $J=7.3,3.4 \mathrm{~Hz}, 1 \mathrm{H}), 2.04-1.82(\mathrm{~m}, 4$ H), 1.53 (ddd, $J=13.3,7.3,3.1 \mathrm{~Hz}, 1 \mathrm{H}$ ).

${ }^{13} \mathrm{C} \mathrm{NMR}\left(75 \mathrm{MHz}, \mathrm{CDCl}_{3}\right): \delta=217.3(\mathrm{C}), 169.6(\mathrm{C}), 124.6(\mathrm{CH}), 124.0$ $(\mathrm{CH}), 56.2(\mathrm{C}), 52.4(\mathrm{CH}), 42.9\left(\mathrm{CH}_{2}\right), 38.7\left(\mathrm{CH}_{2}\right), 36.2\left(\mathrm{CH}_{2}\right), 32.5\left(\mathrm{CH}_{2}\right)$, $26.5\left(\mathrm{CH}_{2}\right), 24.3\left(\mathrm{CH}_{2}\right), 19.7\left(\mathrm{CH}_{2}\right)$.

\section{1',6',9',9a'-Tetrahydro-2'H,4'H-spiro[indene-2,3'-quinolizine]-} $1, \mathbf{4}^{\prime}(\mathbf{3 H})$-dione (9iaa)

To a one-necked round-bottomed flask, equipped with a magnetic stirring bar under an argon atmosphere, were introduced spiro compound 8iaa ( $53 \mathrm{mg}, 0.16 \mathrm{mmol}$ ) in $\mathrm{CH}_{2} \mathrm{Cl}_{2}(5.6 \mathrm{~mL}$ ), followed by Grubbs II catalyst ( $7.13 \mathrm{mg}, 5 \mathrm{~mol} \%$ ). The mixture was stirred at r.t. for $4 \mathrm{~h}$ (the reaction was monitored by TLC). The mixture was then filtered and was purified by flash chromatography on silica gel. The two diastereomers were separated.

First fraction: one diastereomer was isolated as a brown oil $(12 \mathrm{mg}$ $39 \%) ; R_{f}=0.3\left(\mathrm{Et}_{2} \mathrm{O} / \mathrm{PE} ; 8: 2\right)$.

${ }^{1} \mathrm{H} \mathrm{NMR}\left(400 \mathrm{MHz}, \mathrm{CDCl}_{3}\right): \delta=8.05(\mathrm{~d}, J=7.8 \mathrm{~Hz}, 1 \mathrm{H}), 7.47$ (td, $J=7.5$, $1.2 \mathrm{~Hz}, 1 \mathrm{H}$ ), 7.30 (t, $J=7.6 \mathrm{~Hz}, 1 \mathrm{H}$ ), 7.23 (d, J = 7.6 Hz, $1 \mathrm{H}$ ), 5.79 (ddd, $J=9.7,5.5,1.9 \mathrm{~Hz}, 1 \mathrm{H}), 5.71(\mathrm{dd}, J=10.4,1.4 \mathrm{~Hz}, 1 \mathrm{H}), 4.75(\mathrm{~d}, J=18.9$ $\mathrm{Hz}, 1 \mathrm{H}), 3.69(\mathrm{td}, J=10.5,4.1 \mathrm{~Hz}, 1 \mathrm{H}), 3.59$ (ddd, $J=18.8,3.9,2.0 \mathrm{~Hz}$, $1 \mathrm{H}), 3.03(\mathrm{dd}, J=11.6,6.3 \mathrm{~Hz}, 1 \mathrm{H}), 2.96(\mathrm{dd} J=15.4,7.7 \mathrm{~Hz}, 1 \mathrm{H})$, 2.33-2.23 ( $\mathrm{m}, 1 \mathrm{H}), 2.22-2.12(\mathrm{~m}, 1 \mathrm{H}), 2.06$ (dd, $J=18.6,2.4 \mathrm{~Hz}, 1 \mathrm{H}$ ), 2.02-1.95 (m, $1 \mathrm{H}), 1.89$ (ddd, $J=13.8,11.0,3.1 \mathrm{~Hz}, 1 \mathrm{H}$ ), 1.69-1.59 (m, $1 \mathrm{H})$.

${ }^{13} \mathrm{C}$ NMR (101 MHz, $\mathrm{CDCl}_{3}$ ): $\delta=197.55$ (C), 169.65 (C), 143.28 (C), $133.61(\mathrm{CH}), 131.34(\mathrm{C}), 128.73(\mathrm{CH}), 128.37(\mathrm{CH}), 126.89(\mathrm{CH})$ $124.84(\mathrm{CH}), 124.37(\mathrm{CH}), 53.92(\mathrm{C}), 52.48(\mathrm{CH}), 43.29\left(\mathrm{CH}_{2}\right), 32.74$ $\left(\mathrm{CH}_{2}\right), 25.59\left(\mathrm{CH}_{2}\right), 25.20\left(\mathrm{CH}_{2}\right), 23.56\left(\mathrm{CH}_{2}\right)$.

Second fraction: one diastereomer was isolated as a brown oil $(10 \mathrm{mg}$, $33 \%) ; R_{f}=0.2\left(\mathrm{Et}_{2} \mathrm{O} / \mathrm{PE}, 8: 2\right)$.

${ }^{1} \mathrm{H}$ NMR $\left(400 \mathrm{MHz}, \mathrm{CDCl}_{3}\right): \delta=7.77(\mathrm{~d}, J=7.7 \mathrm{~Hz}, 1 \mathrm{H}), 7.59(\mathrm{t}, J=7.4$ $\mathrm{Hz}, 1 \mathrm{H}), 7.45(\mathrm{~d}, J=7.7 \mathrm{~Hz}, 1 \mathrm{H}), 7.38(\mathrm{t}, J=7.4 \mathrm{~Hz}, 1 \mathrm{H}), 5.80$ (ddd, $J=$ 9.9, 5.0, 2.3 Hz, $1 \mathrm{H}), 5.72(\mathrm{dd}, J=10.2,2.4 \mathrm{~Hz}, 1 \mathrm{H}), 4.69(\mathrm{~d}, J=19.3 \mathrm{~Hz}$, $1 \mathrm{H}), 3.80-3.69(\mathrm{~m}, 2 \mathrm{H}), 3.59$ (dd, $J=19.1,2.3 \mathrm{~Hz}, 1 \mathrm{H}), 3.00(\mathrm{~d}, J=$ $16.9 \mathrm{~Hz}, 1 \mathrm{H}), 2.55-2.46(\mathrm{~m}, 1 \mathrm{H}), 2.27-2.13(\mathrm{~m}, 4 \mathrm{H}), 1.90-1.84(\mathrm{~m}, 1$ $\mathrm{H})$.

${ }^{13} \mathrm{C}$ NMR (101 MHz, $\left.\mathrm{CDCl}_{3}\right): \delta=205.41$ (C), 169.51 (C), 153.49 (C), $135.52(\mathrm{C}), 135.19(\mathrm{C}), 127.79(\mathrm{CH}), 126.46(\mathrm{CH}), 124.96(\mathrm{CH}), 124.47$ $(\mathrm{CH}), 123.89(\mathrm{CH}), 56.65(\mathrm{CH}), 53.03(\mathrm{CH}), 42.88\left(\mathrm{CH}_{2}\right), 41.11\left(\mathrm{CH}_{2}\right)$, $33.15\left(\mathrm{CH}_{2}\right), 28.96\left(\mathrm{CH}_{2}\right), 25.55\left(\mathrm{CH}_{2}\right)$.

\section{1',3,4,6',9',9a'-Hexahydro-1H,2'H,4'H-spiro[naphthalene-2,3'-} quinolizine]-1,4'-dione (9jaa)

To a one-necked round-bottomed flask, equipped with magnetic stirring bar under an argon atmosphere, were introduced spiro compound 8jaa $(105 \mathrm{mg}, 0.33 \mathrm{mmol})$ in $\mathrm{CH}_{2} \mathrm{Cl}_{2}(10 \mathrm{~mL})$, followed by Grubbs II catalyst ( $14.3 \mathrm{mg}, 5 \mathrm{~mol} \%$ ). The mixture was stirred at r.t. for $4 \mathrm{~h}$ (the reaction was monitored by TLC). The mixture was then filtered and purified by flash chromatography on silica gel. The two diastereomers were separated.

First fraction: one diastereomer was isolated as a brown solid ( $21 \mathrm{mg}$, $32 \%) ; R_{f}=0.41\left(\mathrm{Et}_{2} \mathrm{O} / \mathrm{PE}, 9: 1\right)$.

${ }^{1} \mathrm{H}$ NMR $\left(300 \mathrm{MHz}, \mathrm{CDCl}_{3}\right): \delta=8.03(\mathrm{~d}, J=7.8 \mathrm{~Hz}, 1 \mathrm{H}), 7.46(\mathrm{t}, J=7.5$ $\mathrm{Hz}, 1 \mathrm{H}), 7.30$ (d, $J=7.3 \mathrm{~Hz}, 1 \mathrm{H}), 7.22(\mathrm{~d}, J=7.6 \mathrm{~Hz}, 1 \mathrm{H}), 5.79(\mathrm{dd}, J=$ $12.1,3.3 \mathrm{~Hz}, 1 \mathrm{H}), 5.69$ (d, $J=10.3 \mathrm{~Hz}, 1 \mathrm{H}), 4.74(\mathrm{~d}, J=18.8 \mathrm{~Hz}, 1 \mathrm{H})$, $3.67(\mathrm{dd}, J=10.3,5.1 \mathrm{~Hz}, 1 \mathrm{H}), 3.57(\mathrm{~d}, J=18.9 \mathrm{~Hz}, 1 \mathrm{H}), 2.99(\mathrm{dt}, J=$ $16.0,7.1 \mathrm{~Hz}, 3 \mathrm{H}), 2.7-1.91(\mathrm{~m}, 6 \mathrm{H}), 1.63(\mathrm{dd}, J=13.9,6.8 \mathrm{~Hz}, 1 \mathrm{H})$.

${ }^{13} \mathrm{C}$ NMR (75 MHz, $\mathrm{CDCl}_{3}$ ): $\delta=197.3$ (C), $169.4(\mathrm{C}), 143.1(\mathrm{C}), 133.4$ $(\mathrm{CH}), 131.1(\mathrm{C}), 128.6(\mathrm{CH}), 128.1(\mathrm{CH}), 126.6(\mathrm{CH}), 124.5(\mathrm{CH}), 124.3$ $(\mathrm{CH}), 53.7(\mathrm{CH}), 52.3(\mathrm{C}), 43.1\left(\mathrm{CH}_{2}\right), 32.5\left(\mathrm{CH}_{2}\right), 32.5\left(\mathrm{CH}_{2}\right), 25.4\left(\mathrm{CH}_{2}\right)$, $25.0\left(\mathrm{CH}_{2}\right), 23.3\left(\mathrm{CH}_{2}\right)$.

Second fraction: one diastereomer was isolated as a brown solid (2.5 $\mathrm{mg}, 38 \%) ; \mathrm{mp} 128^{\circ} \mathrm{C} ; R_{f}=0.25\left(\mathrm{Et}_{2} \mathrm{O} / \mathrm{PE}, 9: 1\right)$. 
${ }^{1} \mathrm{H} \mathrm{NMR}\left(400 \mathrm{MHz}, \mathrm{CDCl}_{3}\right): \delta=8.05(\mathrm{~d}, J=7.4 \mathrm{~Hz}, 1 \mathrm{H}), 7.47(\mathrm{td}, J=7.5$, $1.1 \mathrm{~Hz}, 1 \mathrm{H}), 7.30(\mathrm{t}, J=7.5 \mathrm{~Hz}, 1 \mathrm{H}), 7.23(\mathrm{~d}, J=7.6 \mathrm{~Hz}, 1 \mathrm{H}), 5.82-5.76$ $(\mathrm{m}, 1 \mathrm{H}), 5.72(\mathrm{dd}, J=10.5,1.5 \mathrm{~Hz}, 1 \mathrm{H}), 4.76(\mathrm{~d}, J=18.8 \mathrm{~Hz}, 1 \mathrm{H}), 3.69$ (td, $J=10.4,4.1 \mathrm{~Hz}, 1 \mathrm{H}), 3.59(\mathrm{dd}, J=18.9,1.7 \mathrm{~Hz}, 1 \mathrm{H}), 3.03(\mathrm{dd}, J=$ 11.5, $6.0 \mathrm{~Hz}, 2 \mathrm{H}), 2.97$ (dd, $J=15.4,7.6 \mathrm{~Hz}, 1 \mathrm{H}), 2.33-2.12(\mathrm{~m}, 4 \mathrm{H})$, 2.06 (dd, $J=15.3,2.3 \mathrm{~Hz}, 1 \mathrm{H}$ ), 2.03-1.94 (m, $1 \mathrm{H}$ ), 1.89 (ddd, $J=13.8$, 11.0, $3.1 \mathrm{~Hz}, 1 \mathrm{H})$.

${ }^{13} \mathrm{C}$ NMR (101 MHz, $\mathrm{CDCl}_{3}$ ): $\delta=197.41$ (C), 169.51 (C), 143.15 (C), $133.47(\mathrm{CH}), 131.22(\mathrm{C}), 128.59(\mathrm{CH}), 128.25(\mathrm{CH}), 126.76(\mathrm{CH})$ $124.72(\mathrm{CH}), 124.23(\mathrm{CH}), 53.79(\mathrm{C}), 52.34(\mathrm{CH}), 43.15\left(\mathrm{CH}_{2}\right), 32.61$ $\left(\mathrm{CH}_{2}\right), 29.70\left(\mathrm{CH}_{2}\right), 25.47\left(\mathrm{CH}_{2}\right), 25.07\left(\mathrm{CH}_{2}\right), 23.43\left(\mathrm{CH}_{2}\right)$.

\section{Acknowledgment}

H. Habib-Zahmani warmly thanks Universidad Complutense de Madrid and Region PACA for funding her post-doctoral grant. AixMarseille Université and Université d'Oran-1 are also acknowledged for financial support.

(1) (a) Ley, S. V.; Baxendale, I. R.; Bream, R. N.; Jackson, P. S.; Leach, A. G.; Longbottom, D. A.; Nesi, M.; Scott, J. S.; Storer, R. I.; Taylor, S. J. J. Chem. Soc., Perkin Trans. 1 2000, 3815. (b) Kirschning, A.; Monenschein, A.; Wittenberg, R. Angew. Chem. Int. Ed. 2001, 40, 650.

(2) For some recent reviews, see: (a) Puglisi, A.; Benaglia, M.; Porta, R.; Coccia, F. Curr. Organocat. 2015, 2, 79. (b) Atodiresei, I.; Vila, C.; Rueping, M. ACS Catal. 2015, 5, 1972.

(3) (a) Clapham, B.; Reger, T. S.; Janda, K. D. Tetrahedron 2001, 57 4637. (b) Benaglia, M.; Puglisi, A.; Cozzi, F. Chem. Rev. 2003, 103 3401. (c) Anwander, R. Immobilization of Molecular Catalysts, In Handbook of Heterogeneous Catalysis, 2nd ed., Vol. 1; Ertl, G. Knözinger, H.; Schüth, F.; Weitkamp, J., Eds.; Wiley-VCH: Weinheim, 2008, 583-613. (d) Yamaguchi, K.; Mizuno, N. Synlett 2010, 2365. (e) Rodríguez-Escrich, C.; Pericàs, M. A. Eur. J. Org. Chem. 2015, 1173.

(4) (a) Iijima, K.; Fukuda, W.; Tomoi, M. J. Macromol. Sci. Pure Appl. Chem. 1992, A29, 249. (b) Xu, W.; Mohan, R.; Morrissey, M. M. Tetrahedron Lett. 1997, 38, 7337.

(5) (a) Bernard, M.; Ford, W. T. J. Org. Chem. 1983, 48, 326. (b) Westman, J. Org. Lett. 2000, 3, 3745. (c) Matsukawa, S.; Fukazawa, K.; Kimura, J. RSC Adv. 2014, 4, 27780.

(6) Schwesinger, R.; Willaredt, J.; Schlemper, H.; Keller, M.; Schmitt, D.; Fritz, H. Chem. Ber. 1994, 127, 2435.

(7) (a) Brain, C. T.; Brunton, S. A. Synlett 2001, 382. (b) Graybill, T. L.; Thomas, S.; Wang, M. A. Tetrahedron Lett. 2002, 43, 5305. (c) Adams, G. L.; Graybill, T. L.; Sanchez, R. M.; Magaard, V. W.; Burton, G.; Rivero, R. A. Tetrahedron Lett. 2003, 44, 5041. (d) Legrand, O. Synlett 2000, 752. (e) See also a special issue on strong and hindered bases: Chemfiles 2003, 3, No. 1.

(8) Bensa, D.; Constantieux, T.; Rodriguez, J. Synthesis 2004, 923.

(9) (a) Coelho, A.; El-Maatougui, A.; Ravina, E.; Cavaleiro, J. A. S.; Silva, A. M. S. Synlett 2006, 3324. (b) Vedantham, P.; Guerra, J. M.; Schoenen, F.; Huang, M.; Gor, P. J.; Georg, G. I.; Wang, J. L.;
Neuenswander, B.; Lushington, G. H.; Mitscher, L. A.; Ye, Q-Z.; Hanson, P. R. J. Comb. Chem. 2008, 10, 185. (c) Shao, Y.; Cole, A G.; Brescia, M-R.; Qin, L-Y.; Duo, J.; Stauffer, T. M.; Rokosz, L. L.; McGuinness, B. F.; Henderson, I. Bioorg. Med. Chem. Lett. 2009 , 19, 1399. (d) Baumann, M.; Baxendale, I. R.; Brasholz, M.; Hayward, J. J.; Ley, S. V.; Nikbin, N. Synlett 2011, 1375.

(10) (a) Ballini, R.; Barboni, L.; Castrica, L.; Fringuelli, F.; Lanari, D.; Pizzo, F.; Vaccaro, L. Adv. Synth. Catal. 2008, 350, 1218 (b) Bonollo, S.; Lanari, D.; Longo, J. M.; Vaccaro, L. Green Chem. 2012, 14, 164.

(11) (a) Angelini, T.; Fringuelli, F.; Lanari, D.; Pizzo, F.; Vaccaro, L. Tetrahedron Lett. 2010, 51, 1566. (b) Zvagulis, A.; Bonollo, S.; Lanari, D.; Pizzo, F.; Vaccaro, L. Adv. Synth. Catal. 2010, 352, 2489.

(12) Angelini, T.; Ballerini, E.; Bonollo, S.; Curini, M.; Lanari, D. Green Chem. Lett. Rev. 2014, 7, 11.

(13) Angelini, T.; Bonollo, S.; Lanari, D.; Pizzo, F.; Vaccaro, L. Org. Biomol. Chem. 2013, 11, 5042.

(14) (a) Wack, H.; Taggi, A. E.; Hafez, A. M.; Drury, W. J. III; Lectka, T. J. Am. Chem. Soc. 2001, 123, 1531. (b) Taggi, A. E.; Hafez, A. M.; Wack, H.; Young, B.; Ferraris, D.; Lectka, T. J. Am. Chem. Soc 2002, 124, 6626. (c) Pilling, A. W.; Boehmer, J.; Dixon, D. J. Angew. Chem. Int. Ed. 2007, 46, 5428. (d) Pilling, A. W.; Boehmer, J.; Dixon, D. J. Chem. Commun. 2008, 832. (e) Yang, T.; Ferrali, A.; Campbell, L.; Dixon, D. J. Chem. Commun. 2008, 2923. (f) Bogle, K. M.; Hirst, D. J.; Dixon, D. J. Tetrahedron 2010, 66, 6399. (g) Muratore, M. E.; Shi, L.; Pilling, A. W.; Storer, R. I.; Dixon, D. J. Chem. Commun. 2012, 48, 6351.

(15) (a) Bonne, D.; Coquerel, Y.; Constantieux, T.; Rodriguez, J. Tetrahedron: Asymmetry 2010, 21, 1085. (b) Bonne, D.; Constantieux, T.; Coquerel, Y.; Rodriguez, J. Chem. Eur. J. 2013, 19, 2218. (c) Bugaut, X.; Bonne, D.; Coquerel, Y.; Rodriguez, J.; Constantieux, T. Curr. Org. Chem. 2013, 17, 1920. (d) Allais, C.; Grassot, J-M.; Rodriguez, J.; Constantieux, T. Chem. Rev. 2014, 114, 10829. (e) Bugaut, X.; Constantieux, T.; Coquerel, Y.; Rodriguez, J. 1,3-Dicarbonyls in Multicomponent Reactions, In Multicomponent Reactions in Organic Synthesis; Zhu, J.; Wang, Q.; Wang, M., Eds.; Wiley-VCH: Weinheim, 2014, Chap. 5, 109.

(16) Kim, J. J.; Wood, M. R.; Stachel, S. J.; de Leon, P.; Nomland, A.; Stump, C. A.; McWherter, M. A.; Schirripa, K. M.; Moore, E. L. Salvatore, C. A.; Selnick, H. G. Bioorg. Med. Chem. Lett. 2014, 24, 258.

(17) (a) Jang, J-H.; Asami, Y.; Jang, J-P.; Kim, S-O.; Moon, D. O.; Shin, K-S.; Hashizume, D.; Muroi, M.; Saito, T.; Oh, H.; Kim, B. Y.; Osada, H.; Ahn, J. S. J. Am. Chem. Soc. 2011, 133, 6865. (b) Lesma, G.; Cecchi, R.; Cagnotto, A.; Gobbi, M.; Meneghetti, F.; Musolino, M.; Sacchetti, A.; Silvani, A. J. Org. Chem. 2013, 78, 2600.

(18) de Almeida Leone, P.; Carroll, A. R.; Towerzey, L; King, G.; McArdie, B. M.; Kern, G.; Fisher, S.; Hooper, J. N. A.; Quinn, R. J. Org. Lett. 2008, 10, 2585.

(19) Horn, W. S.; Simmonds, M. S. J.; Schwartz, R. E.; Blaney, W. M. Tetrahedron 1995, 51, 3969.

(20) (a) Kotha, S.; Deb, A. C.; Lahiri, K.; Manivannan, E. Synthesis 2008, 165. (b) Marson, C. M. Chem. Soc. Rev. 2011, 40, 5514. (c) Zheng, Y.; Tice, C. M.; Singh, S. B. Bioorg. Med. Chem. Lett. 2014, 24, 3673.

(21) See, for example: (a) Khan, F. A.; Dash, J. J. Org. Chem. 2003, 68 , 4556. (b) Hilmey, D. G.; Paquette, L. A. Org. Lett. 2005, 7, 2067. (c) Presset, M.; Coquerel, Y.; Rodriguez, J. Org. Lett. 2010, 12, 4212. (d) Kang, F-A.; Sui, Z. Tetrahedron Lett. 2011, 52, 4204. (e) Gálvez, J.; Castillo, J.-C.; Quiroga, J.; Rajzmann, M. 
Rodriguez, J.: Coquerel, Y. Org. Lett. 2014, 16, 4126. (f) Yang. W.; Sun, X.; Wembo, Y.; Rachita, R.; Deschamps, J. R.; Mitchell, L. A.; Jiang, C.; MacKerell, A. D. Jr.; Xue, F. Org. Lett. 2015, 17, 3070.

(22) Cossy, J.; Bouzide, A.; Leblanc, C. J. Org. Chem. 2000, 65, 7257.

(23) Zhou, C-Y.; Che, C-M. J. Am. Chem. Soc. 2007, 129, 5828.

(24) (a) Li, M.; Dixon, D. J. Org. Lett. 2010, 12, 3784. (b) Li, M.; Hawkins, A.; Barber, D. M.; Bultinck, P.; Herrebout, W.; Dixon, D. J. Chem. Commun. 2013, 49, 5265.

(25) (a) Habib-Zahmani, H.; Viala, J.; Hacini, S.; Rodriguez, J. Synlett 2007, 1037. (b) Sternativo, S.; Battistelli, B.; Bagnoli, L.; Santi, C.; Testaferri, L.; Marini, F. Tetrahedron Lett. 2013, 54, 6755.

(26) Boddaert, T.; Coquerel, Y.; Rodriguez, J. Adv. Synth. Catal. 2009, $351,1744$.

(27) For previous synthesis and characterization of these bicyclic ketones through the same strategy, see: Filippini, M.-H.; Faure, R.; Rodriguez, J. J. Org. Chem. 1995, 60, 6872.
(28) For the synthesis of cyclic 1,3-ketoamides $\mathbf{1}$, see the Supporting Information.

29) Reaction of substrate 1f with acrolein also afforded the desired spiro compound $\mathbf{3 f a}$, but this product was obtained as a mixture with a bicyclic compound $\mathbf{4 f a}$ resulting from a Michael addition/intramolecular aldolization sequence. For more details, see the Supporting Information.

(30) Diastereomeric ratios have been determined from the NMR analysis of the crude product, using quantitative ${ }^{13} \mathrm{C}$ NMR technic, with long relaxation delays.

(31) CCDC 1429887 (3ed), CCDC 1429886 (5ld), and CCDC 1430278 (8fb) contain the supplementary crystallographic data for this paper. These data can be obtained free of charge from The Cambridge Crystallographic Data Centre via www.ccdc.cam.ac.uk/getstructures. 